\title{
QUEEN'S
UNIVERSITY
BELFAST
}

\section{Electronic polarizability as the fundamental variable in the dielectric properties of two-dimensional materials}

Tian, T., Scullion, D., Hughes, D., Hua Li, L., Shih, C-J., Coleman, J., Chhowalla, M., \& Santos, E. J. G. (2019). Electronic polarizability as the fundamental variable in the dielectric properties of two-dimensional materials. Nano Letters. https://doi.org/10.1021/acs.nanolett.9b02982

\section{Published in:}

Nano Letters

\section{Document Version:}

Peer reviewed version

Queen's University Belfast - Research Portal:

Link to publication record in Queen's University Belfast Research Portal

\section{Publisher rights}

(c) 2019 American Chemical Society. This work is made available online in accordance with the publisher's policies. Please refer to any applicable terms of use of the publisher.

\section{General rights}

Copyright for the publications made accessible via the Queen's University Belfast Research Portal is retained by the author(s) and / or other copyright owners and it is a condition of accessing these publications that users recognise and abide by the legal requirements associated with these rights.

Take down policy

The Research Portal is Queen's institutional repository that provides access to Queen's research output. Every effort has been made to ensure that content in the Research Portal does not infringe any person's rights, or applicable UK laws. If you discover content in the Research Portal that you believe breaches copyright or violates any law, please contact openaccess@qub.ac.uk. 


\title{
Electronic polarizability as the fundamental
}

\section{variable in the dielectric properties of two-dimensional materials}

\author{
Tian Tian, ${ }^{\dagger, \perp}$ Declan Scullion, ${ }^{\ddagger, \perp}$ Dale Hughes, ${ }^{\ddagger}$ Lu Hua Li, ${ }^{\llbracket}$ Chih-Jen \\ Shih,${ }^{\dagger}$ Jonathan Coleman, $§$ Manish Chhowalla, ${ }^{\|}$and Elton J. G. Santos ${ }^{*, \downarrow}$ \\ $\dagger$ †nstitute for Chemical and Bioengineering, ETH Zürich, Vladimir Prelog Weg 1, CH-8093 \\ Zürich, Switzerland \\ $\ddagger$ School of Mathematics and Physics, Queen’s University Belfast, BT7 INN, United Kingdom \\ 9Institute for Frontier Materials, Deakin University, Victoria, VIC 3216, Australia \\ $\S$ School of Physics, Centre for Research on Adaptive Nanostructures and Nanodevices (CRANN) \\ and Advanced Materials and BioEngineering Research (AMBER), Trinity College Dublin, Dublin \\ 2, Ireland \\ ||Department of Materials Science \& Metallurgy, University of Cambridge, CB3 OFS, United \\ Kingdom
}

$\perp T$. T. and D. S. contributed equally to this work

E-mail: e.santos@qub.ac.uk 


\begin{abstract}
The dielectric constant, which defines the polarization of the media, is a key quantity in condensed matter. It determines several electronic and optoelectronic properties important for a plethora of modern technologies from computer memory to field effect transistors and communication circuits. Moreover, the importance of the dielectric constant in describing electromagnetic interactions through screening plays a critical role in understanding fundamental molecular interactions. Here we show that despite its fundamental transcendence, the dielectric constant does not define unequivocally the dielectric properties of two-dimensional (2D) materials due to the locality of their electrostatic screening. Instead, the electronic polarizability correctly captures the dielectric nature of a 2D material which is united to other physical quantities in an atomically thin layer. We reveal a long-sought universal formalism where electronic, geometrical and dielectric properties are intrinsically correlated through the polarizability opening the door to probe quantities yet not directly measurable including the real covalent thickness of a layer. We unify the concept of dielectric properties in any material dimension finding a global dielectric anisotropy index defining their controllability through dimensionality.
\end{abstract}

\title{
Keywords
}

Dielectric screening, electronic polarizability, two-dimensional material, scaling relation, first principles simulations, dielectric anisotropy 


\section{Introduction}

The dielectric constant $\varepsilon$ (also known as the relative permittivity) plays a crucial role in bridging various fundamental material properties, such as bandgap, ${ }^{1,2}$ optical absorption ${ }^{3}$ and conductivity ${ }^{4}$ with elemental interactions. The central place of $\varepsilon$ in solid-state physics drives the analysis of several phenomena where is common to classify a material accordingly to its ability to screen an electric field $\boldsymbol{E}$ in terms of insulators, metals and semiconductors. Such definitions determine a broad range of condensed matter physics, as well as in related fields in chemistry and materials science. The ability to compute and measure $\varepsilon$ in bulk materials is well established via different theoretical $^{5,6}$ and experimental techniques ${ }^{7}$ of distinct flavors where the probe of the dielectric properties is made through an external electric field. Despite its obvious appeal, however, it is still unknown whether such quantity can determine the electronic and dielectric properties of twodimensional (2D) materials. ${ }^{8}$ The confined nature of such atomically-thin $2 \mathrm{D}$ crystals associated with the attenuated and anisotropic character of the dielectric screening ${ }^{9-15}$ has generated longstanding debates whether the dielectric constant truly represents the dielectric features of such low-dimensional systems. The controversy of values reported by both theoretical and experimental approaches can be widely seen throughout the specialized literature, see Ref. ${ }^{16}$ for a summary, where the variation of $\varepsilon$ can be more than one order of magnitude. As a consequence, several key physical parameters that scale with $\varepsilon$, such as the exciton binding energy and Debye screening length, cannot be reliably estimated due to the discrepancy of the reported magnitudes of $\varepsilon$.

Here, by using a combination of analytical and numerical models liaised with highly-accurate first-principles methods involving high-throughput screening techniques, we show that the dielectric constant does not provide a reliable description of the screening features of a 2D material. The interplay between local electrostatic interactions in the monolayer and the volume dependence in the definition of $\varepsilon$ makes such quantity questionable. We propose however that the electronic polarizability that describes the electron dipole in the 2D material as the true descriptor of its dielectric nature. We overcome several problems intrinsic to thin layers not achievable using conventional effective dielectric medium models, such as the real thickness of a monolayer and any dependence 
on the long-range Coulomb potential. We unveil universal scaling relations between electronic and dielectric properties through the electronic polarizability, such as band gaps, optical spectra and exciton radius, for the current library of known 2D materials involving different lattice symmetries, atomic elements and chemical and physical properties. Moreover, the concept of electronic polarizabilities bridges the gap between the dielectric properties of $2 \mathrm{D}$ and $3 \mathrm{D}$ systems through a novel dielectric anisotropy index that generalized the concept of dielectric control using dimensionality and bandgap. Our results open a new avenue for the study of the dielectric properties of 2D compounds using techniques yet to be explored.

\section{Results and discussions}

\section{Lattice-dependency of macroscopic dielectric constant}

We first approach the discrepancy of macroscopic dielectric constant of 2D materials, by showing that the current definition of $\varepsilon$ used in layered materials is ill-defined. This can be viewed in a model system as illustrated in Figure 1, where an isolated 2D material is placed in the $x y$-plane of a periodically repeating superlattice (SL) with a length $L$ along the $z$-direction separating the cell images. The static macroscopic dielectric tensor from the superlattice $\varepsilon_{\mathrm{SL}}^{p q}$, is determined through fundamental electrostatics by the response of the polarization density $\boldsymbol{P}^{p}$ under small perturbative external field $\boldsymbol{E}^{q}$, where $p, q$ determine their directions, respectively: ${ }^{4}$

$$
\begin{aligned}
\varepsilon_{\mathrm{SL}}^{p q} & =\kappa^{p q}+\frac{\partial \boldsymbol{P}^{p}}{\varepsilon_{0} \partial \boldsymbol{E}^{q}} \\
\boldsymbol{P}^{p} & =\frac{\boldsymbol{u}^{p}}{\Omega}=\frac{\int_{\mathrm{SL}} \rho(\boldsymbol{r}) \boldsymbol{r}^{p} d^{3} \boldsymbol{r}}{A L}
\end{aligned}
$$

where $\kappa$ is the dielectric tensor of the environment, $\boldsymbol{u}$ is the total dipole moment within the SL, $\rho$ is the spatial charge density, $\Omega=A L$ is the volume of the supercell, $A$ is the $x y$-plane area of the $\mathrm{SL}$ and $\varepsilon_{0}$ is vacuum permittivity. Here we limit our study on the electronic contributions to 
the macroscopic dielectric constant where the dipole $\boldsymbol{P}$ results from the response of the electron density under an external field. Ionic contributions ${ }^{17}$ to $\varepsilon_{\mathrm{SL}}$ have previously been shown to be negligible ${ }^{18}$ and are not considered here. The symmetry of $2 \mathrm{D}$ materials leads to inappreciable off-diagonal elements of the dielectric tensor $(p \neq q)$, while the diagonal elements $\varepsilon_{\mathrm{SL}}^{x x}, \varepsilon_{\mathrm{SL}}^{y y}$ and $\varepsilon_{\mathrm{SL}}^{z z}$ can be different. ${ }^{19}$ Considering that the $2 \mathrm{D}$ material is placed in vacuum $\left(\kappa^{p p}=1\right.$ and $\left.\kappa^{p q}=0\right)$, we can distinguish two components of $\varepsilon_{\mathrm{SL}}$, namely the in-plane $\left(\varepsilon_{\mathrm{SL}}^{\|}\right)$and out-of-plane $\left(\varepsilon_{\mathrm{SL}}^{\perp}\right)$ dielectric constants, where $\varepsilon_{\mathrm{SL}}^{\|}=\left(\varepsilon_{\mathrm{SL}}^{x x}+\varepsilon_{\mathrm{SL}}^{y y}\right) / 2$ and $\varepsilon_{\mathrm{SL}}^{\perp}=\varepsilon_{\mathrm{SL}}^{z z}$. The absence of bonding perpendicular to the plane confines the induced dipole moments along the $z$-direction within a range of $\sim 5-6 \AA$ into the vacuum (Figure 1a and Supplementary Figure 1). Under a given external field, the strong confinement of the induced dipole moment $u$ causes the integral in the numerator of Eq. $1 \mathrm{~b}$ to be converged within few Åss resulting that the dipole moment from the periodic supercell images do not mutually interfere.

Conversely, the increase of $L$ in the denominator of Eq. $1 \mathrm{~b}$ dilutes the polarization density, and in turn makes both $\varepsilon_{\mathrm{SL}}^{\|}$and $\varepsilon_{\mathrm{SL}}^{\perp}$ dropping to unity when $L$ is infinitely large, which is not physical. Despite the simplicity of this argument, any calculation performed using such definition will intrinsically depend on the magnitude of $L$, an artificial parameter introduced by the simulation setup. This dependence can be clearly demonstrated by plotting $\varepsilon_{\mathrm{SL}}^{\|}$and $\varepsilon_{\mathrm{SL}}^{\perp}$ calculated from density functional theory (DFT) (see Theoretical Methods for details) as a function of $L$ for P $\overline{6} \mathrm{~m} 2$ transition metal dichalcogenides (TMDCs), $2 \mathrm{H}-\mathrm{MX}_{2}$, where $\mathrm{M}=\mathrm{Mo}, \mathrm{W}$ and $\mathrm{X}=\mathrm{S}, \mathrm{Se}$, Te (top panels of Figure $1 \mathbf{b}$ and 1c, respectively). To obtain a better description of the electronic band structure, the calculations of dielectric properties were performed at the level of Heyd-Scuseria-Ernzerhof (HSE06) hybrid functional. ${ }^{20,21}$ Both components of the dielectric constant decrease with $L$ as excepted. To rule out the possibility that the result is affected by the choice of the functional, we performed simulations at higher levels of theory using many-body techniques $\left(\mathrm{G}_{0} \mathrm{~W}_{0}\right)$, which invariably give alike results (see Supplementary Figure 2). The lattice-size dependency also exists for the dielectric function in the frequency domain. 

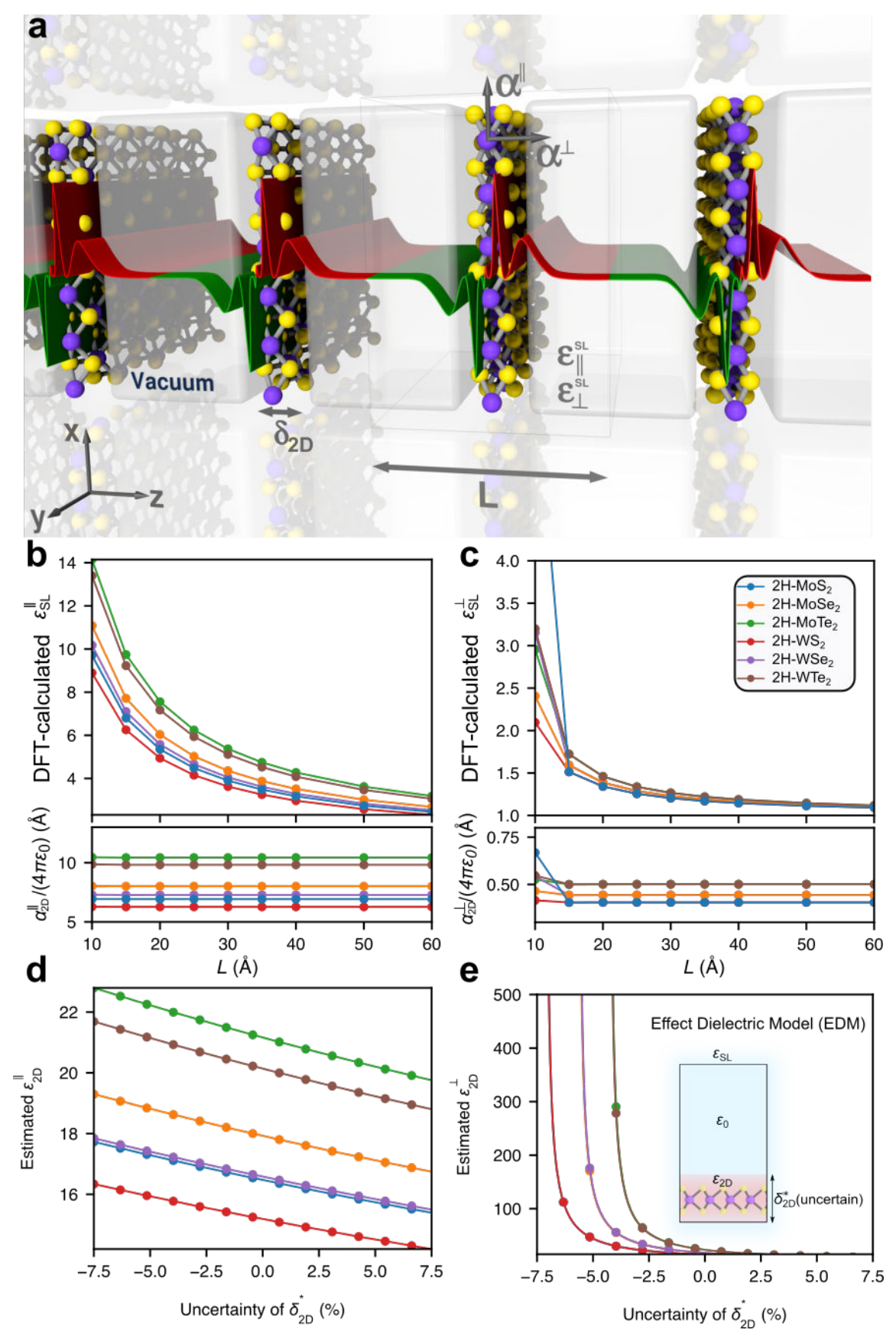

Figure 1: 2D polarizability and the breakdown of effective dielectric model (EDM) a, 3D illustration of the spatial distribution of the charge density change $\Delta \rho(z)$ along the z-direction for monolayer $2 \mathrm{H}-\mathrm{MoS}_{2}$ in a periodic superlattice under external eletric field of $0.01 \mathrm{~V} / \AA$. The green and red regions represent negative and positive induced charges, respectively. The macroscopic $\varepsilon_{\mathrm{SL}}^{\|}$and $\varepsilon_{\mathrm{SL}}^{\perp}$ are influenced by the lattice size $L$, while the 2D polarizabilities $\alpha_{2 \mathrm{D}}^{\|}$and $\alpha_{2 \mathrm{D}}^{\perp}$ are invariant to $L$. b, $\varepsilon_{\mathrm{SL}}^{\|}$(top) and $\alpha_{2 \mathrm{D}}^{\|}$(bottom) as functions of $L$ for the $2 \mathrm{H}$ TMDCs. c, $\varepsilon_{\mathrm{SL}}^{\perp}$ (top) and $\alpha_{2 \mathrm{D}}^{\perp}$ (bottom) as functions of $L$ for the 2H TMDCs. The polarizabilities in $\mathbf{b}$ and $\mathbf{c}$ are constant when $L>15 \AA$, compared with the $L$-dependence of $\varepsilon_{\mathrm{SL}}$. d-e, Estimated $\varepsilon_{2 \mathrm{D}}^{\|}$and $\varepsilon_{2 \mathrm{D}}^{\perp}$, respectively, using EDM as a function of the uncertainty of the effective layer thickness $\delta_{2 \mathrm{D}}^{*}$. The inset in e, shows schematically the main parameters utilized in EMD: the vacuum layer $\left(\varepsilon_{0}\right)$, an approximate thickness of the layer which is given by $\delta_{2 \mathrm{D}}^{*}$, and the obtained $\varepsilon_{2 \mathrm{D}}$. The length of the box perpendicular to the surface of the layer is given by $I 6$ (not shown). Overall, there is a large variation and associated errors to both components of the dielectric constant for small changes of $\delta_{2 \mathrm{D}}^{*}$ in the range of $\pm 7.5 \%$. 
We carried out similar analysis for frequency-dependent $\varepsilon_{\mathrm{SL}}^{\|}(\omega)$ and $\varepsilon_{\mathrm{SL}}^{\perp}(\omega)$ using different approaches including Perdew-Burke-Ernzerhof (PBE) exchange-correlation functional, ${ }^{22-24} \mathrm{G}_{0} \mathrm{~W}_{0}{ }^{25}$ and Bethe-Salpeter equation $\left(\mathrm{G}_{0} \mathrm{~W}_{0}-\mathrm{BSE}\right)^{26}$ (see Supplementary Section S1.2 and Supplementary Figures 3-8). Despite the various levels of theory analyzed and the increased accuracy of the calculated optical properties due to the inclusion of many-body screening and excitonic effects, the magnitude of the dielectric function universally decreases with $L$ over the frequency. The underlying physical reason for such dependence can be noticed in the definition of the dielectric function versus $\omega$ shown in Eqs. S1-S2, which also depend on the volume of the unit cell. These results indicate that any quantity that depends on $\varepsilon_{\mathrm{SL}}^{\|}(\omega)$ and $\varepsilon_{\mathrm{SL}}^{\perp}(\omega)$, such as the optical absorption $\left(\operatorname{Im}\left\{\varepsilon_{\mathrm{SL}}(\omega)\right\}\right)$, refractive index $\left(n=\sqrt{\operatorname{Re}\left\{\varepsilon_{\mathrm{SL}}\right\}}\right)$ and electron energy loss spectrum (EELS, $\left.\operatorname{Im}\left\{-1 / \varepsilon_{\mathrm{SL}}(\omega)\right\}\right)$, suffers the same deficiencies for 2D materials.

\section{The electronic polarizability of 2D materials}

To solve the problem described above, we need to find the $L$-independent alternative of $\varepsilon_{\mathrm{SL}}$, which is related to both electrostatic and optical properties of a $2 \mathrm{D}$ material. ${ }^{27}$ By multiplying Eq. $1 \mathrm{~b}$ with $L$, we obtain the sheet polarization density, that is, $\boldsymbol{\mu}_{2 \mathrm{D}}^{p}=\boldsymbol{u}^{p} / A$, along the direction $p$. Following the discussion in the previous section, $\boldsymbol{\mu}_{2 \mathrm{D}}^{p}$ becomes independent of the lattice size when $L$ is large enough, due to the short decay of the induced charge density $\Delta \rho$ into the vacuum (see Supplementary Figure 1). Similar to the molecular polarizability, ${ }^{28}$ we utilize the concept of electronic polarizability $\alpha_{2 \mathrm{D}}$, which has been used previously to solve exciton-related problems in 2D materials. ${ }^{12,29,30} \alpha_{2 \mathrm{D}}$ is a macroscopic quantity that characterizes the ability to induce dipole moments in a 2D material, and is associated with $\boldsymbol{\mu}_{2 \mathrm{D}}$ through: $\boldsymbol{\mu}_{2 \mathrm{D}}^{p}=\sum_{q} \alpha_{2 \mathrm{D}}^{p q} \overline{\boldsymbol{E}}_{\mathrm{loc}}^{q},{ }^{31}$ where $\overline{\boldsymbol{E}}_{\mathrm{loc}}$ is the cell-averaged "local" electric field acting on the 2D material to induce polarization. ${ }^{32}$ Alike to $\mu_{2 \mathrm{D}}, \overline{\boldsymbol{E}}_{\text {loc }}$ is also a macroscopic quantity that excludes the fields generated by the dipoles of the 2 D sheet from $\boldsymbol{E}$. Note the term "local" in $\overline{\boldsymbol{E}}_{\text {loc }}$ is adapted to resemble the Lorentz model ${ }^{32}$ which has also been used for other low-dimensional materials (e.g. nanotube ${ }^{33}$ and molecules, ${ }^{31}$ and should be distinguished with the microscopic local field $\boldsymbol{E}_{\mathrm{loc}}(\boldsymbol{r})$ which is spatially changing. 
Such macroscopic treatment of polarizability is valid when the length of the superlattice is significantly larger than the spatial distribution of induced charges. At $L \rightarrow \infty$ limit, $\overline{\boldsymbol{E}}_{\text {loc }}$ can be solved using electrostatic boundary conditions of the slab geometry. ${ }^{34,35}$ The continuity of the electric field along the in-plane direction gives $\overline{\boldsymbol{E}}_{\text {loc }}^{\|}=\boldsymbol{E}^{\|}$, while for the out-of-plane component, the dipole screening yields $\overline{\boldsymbol{E}}_{\text {loc }}^{\perp}=\boldsymbol{E}^{\perp}+\boldsymbol{\mu}_{2 \mathrm{D}}^{\perp} / L,{ }^{31,35}$ where $\boldsymbol{E}^{\|}$and $\boldsymbol{E}^{\perp}$ are the external field along the in-plane and out-of-plane directions, respectively. Combining with Eqs. 1a and $1 \mathrm{~b}, \alpha_{2 \mathrm{D}}^{\|}$and $\alpha_{2 \mathrm{D}}^{\perp}$ can be related with $\varepsilon_{\mathrm{SL}}^{\|}$and $\varepsilon_{\mathrm{SL}}^{\perp}$, respectively:

$$
\begin{aligned}
& \varepsilon_{\mathrm{SL}}^{\|}=1+\frac{\alpha_{2 \mathrm{D}}^{\|}}{\varepsilon_{0} L} \\
& \varepsilon_{\mathrm{SL}}^{\perp}=\left(1-\frac{\alpha_{2 \mathrm{D}}^{\perp}}{\varepsilon_{0} L}\right)^{-1}
\end{aligned}
$$

Using these relations, we show that the calculated $\alpha_{2 \mathrm{D}}^{\|}$and $\alpha_{2 \mathrm{D}}^{\perp}$ of the selected TMDCs as a function of $L$ in the bottom panels of Figure $1 \mathrm{~b}$ and $1 \mathrm{c}$, respectively. In contrast to $\varepsilon_{\mathrm{SL}}^{\|}$and $\varepsilon_{\mathrm{SL}}^{\perp}$, we observe that both $\alpha_{2 \mathrm{D}}^{\|}$and $\alpha_{2 \mathrm{D}}^{\perp}$ reach convergence when $L \sim 10 \AA, 15 \AA$, respectively. Such results are in good agreement with the spatially localized induced dipole moment of a 2D material as shown in Supplementary Figure 1. Equations $2 a-2 b$ can also be used to remove the dependence on $L$ for $\varepsilon_{\mathrm{SL}}^{\|}(\omega)$ and $\varepsilon_{\mathrm{SL}}^{\perp}(\omega)$, generating lattice-independent electronic polarizability $\alpha_{\mathrm{SL}}^{\|}(\omega)$ and $\alpha \frac{\perp}{\mathrm{SL}}(\omega)$ in the frequency domain, respectively (see details in Supplementary Section S1.2). These findings indicate that the electronic polarizability $\alpha_{2 \mathrm{D}}$ captures the essence of the dielectric properties of $2 \mathrm{D}$ materials. In contrast to the ill-defined macroscopic $\varepsilon_{\mathrm{SL}}, \alpha_{2 \mathrm{D}}$ has a unique definition, and does not suffer from the dependency on the lattice size. It is worthy mentioning that Eqs.2a-2b were obtained using purely electrostatic arguments without any assumption regarding the medium where the $2 \mathrm{D}$ material is immersed or a capacitance model where an effective dielectric response can be extracted. More details about the choice of the 2D polarizability, comparison with other methods, simulations at the frequency-dependent domain can be found in Supplementary Section S1.2. 


\section{Comparison with the effective dielectric model (EDM)}

Apart from the 2D electronic polarizability proposed here, the effective dielectric model (EDM) is commonly used in literature to treat the 2D material as a slab with an effective dielectric tensor $\varepsilon_{2 \mathrm{D}}$ and thickness $\delta_{2 \mathrm{D}}^{*}$. Such method can be found in both experimental and theoretical studies, such as to interpret ellipsometry data, ${ }^{36-39}$ reflectance / transmission spectra, ${ }^{40,41}$ optical conductance ${ }^{27}$ and many-body interactions ${ }^{19,42}$ of 2D materials. The EDM allows applying physical concepts of bulk systems directly to their $2 \mathrm{D}$ counterparts using $\varepsilon_{2 \mathrm{D}}$. However, there are several drawbacks of such approach. For instance, the wavevector $q$-dependency of dielectric screening in $2 \mathrm{D}$ sheets ${ }^{12,29,43}$ is not captured. More severely, here we show that, due to the uncertainty of $\delta_{2 \mathrm{D}}^{*}$, the calculated $\varepsilon_{2 \mathrm{D}}$, in particular its out-of-plane component, is extremely sensitive to the choice of $\delta_{2 \mathrm{D}}^{*}$, making such model questionable.

The basic assumption of EDM can be seen in the inset of Figure 1e, where the macroscopic $\varepsilon_{\mathrm{SL}}$ is considered to be composed by $(i)$ a $2 \mathrm{D}$ slab with an effective dielectric constant $\varepsilon_{2 \mathrm{D}}$ and a thickness $\delta_{2 \mathrm{D}}^{*}$, and (ii) a vacuum spacing with distance $L-\delta_{2 \mathrm{D}}^{*}$. Using the effective medium theory (EMT), ${ }^{34,44}$ the relation between $\varepsilon_{\mathrm{SL}}$ and $\varepsilon_{2 \mathrm{D}}$ can be expressed using capacitance-like equations: $27,45,46$

$$
\begin{aligned}
& \varepsilon_{\mathrm{SL}}^{\|}=\frac{\delta_{2 \mathrm{D}}^{*}}{L} \varepsilon_{2 \mathrm{D}}^{\|}+\left(1-\frac{\delta_{2 \mathrm{D}}^{*}}{L}\right) \\
& \frac{1}{\varepsilon_{\mathrm{SL}}^{\perp}}=\frac{\delta_{2 \mathrm{D}}^{*}}{L} \frac{1}{\varepsilon_{2 \mathrm{D}}^{\perp}}+\left(1-\frac{\delta_{2 \mathrm{D}}^{*}}{L}\right)
\end{aligned}
$$

In principle, both the values of $\varepsilon_{2 \mathrm{D}}$ and $\delta_{2 \mathrm{D}}^{*}$ are unknown for a certain $2 \mathrm{D}$ material. To minimize the modeling error, we used non-linear least-square fitting to extract $\varepsilon_{2 \mathrm{D}}$ and $\delta_{2 \mathrm{D}}^{*}$ of selected $2 \mathrm{H}$ TMDCs simultaneously from ab initio $\varepsilon_{\mathrm{SL}}-L$ data in Figure 1b and 1c (see details in Supplementary Figure 10). The fitted values of the slab thickness, $\delta_{2 \mathrm{D}}^{\|, \text {fit }}$ and $\delta_{2 \mathrm{D}}^{\perp, \text { fit }}$ from in-plane and out-of-plane data, respectively, are shown in Supplementary Table 1. Although the uncertainty only corresponds to a few percent of the interlayer spacing in the bulk structure of these 2D materials, its influence on the calculated values $\varepsilon_{2 \mathrm{D}}^{\|}$and $\varepsilon_{2 \mathrm{D}}^{\perp}$ is substantial. We estimated the dispersion 
of $\varepsilon_{2 \mathrm{D}}^{\|}$and $\varepsilon_{2 \mathrm{D}}^{\perp}$ considering slightly deviations of $\delta_{2 \mathrm{D}}^{*}$ from the best fitted value by $\pm 7.5 \%$ (Figures 1d and 1e). Strikingly, $\varepsilon_{2 \mathrm{D}}^{\|}$decays linearly with $\delta_{2 \mathrm{D}}^{*}$, while $\varepsilon_{2 \mathrm{D}}^{\perp}$ spans over more than one order of magnitude. The sensitivity of $\varepsilon_{2 \mathrm{D}}^{\perp}$ to $\delta_{2 \mathrm{D}}^{*}$ explains the discrepancy in literature for both isotropic ${ }^{19}$ and highly anisotropic ${ }^{27,45} \varepsilon_{2 \mathrm{D}}$ tensors on 2D materials extracted using EDM. As a consequence, the estimated values of $\varepsilon_{2 \mathrm{D}}$, in particular its out-of-plane component, are highly controversial.

On the contrary, the proposed $\alpha_{2 \mathrm{D}}$ approach does not suffer from such limitations, despite its relatively simple formalism. The relative uncertainty of $\alpha_{2 \mathrm{D}}$ is generally at the order of $10^{-4}$ (Supplementary Figure 9). In addition, the calculation of $\alpha_{2 \mathrm{D}}$ is technically simpler than $\varepsilon_{2 \mathrm{D}}:(i)$ $\alpha_{2 \mathrm{D}}$ can be achieved using single-point calculation of macroscopic dielectric tensor, while $\varepsilon_{2 \mathrm{D}}$ requires non-linear fitting of multiple $\varepsilon_{\mathrm{SL}}-L$ data points; (ii) the values of $\alpha_{2 \mathrm{D}}$ typically converge well for $L(\sim 20 \AA)$, while $\varepsilon_{2 \mathrm{D}}$ suffers from the uncertainties as described above.

\section{Universal scaling laws of $\alpha_{2 \mathrm{D}}$}

For bulk materials, pioneering works from the 1950s had demonstrated empirical equations between $\varepsilon$ and the bandgap $E_{\mathrm{g}}$, including the Moss ${ }^{1,2,47}$ or Ravindra ${ }^{48,49}$ relations. Such universal relations, if exist in the context of $\alpha_{2 \mathrm{D}}$, would be of high importance for studying and predicting the screening of $2 \mathrm{D}$ materials. Inspired by the random phase approximation (RPA) theory ${ }^{5}$ within the $\mathbf{k} \cdot \mathbf{p}$ formalism, ${ }^{3,30}$ we propose the following universal relations for $\alpha_{2 \mathrm{D}}^{\|}$and $\alpha_{2 \mathrm{D}}^{\perp}$, for $2 \mathrm{D}$ materials (see Supplementary Section S3 for details):

$$
\begin{aligned}
& E_{\mathrm{g}}=C^{\|} / \alpha_{2 \mathrm{D}}^{\|} \\
& \hat{\delta}_{2 \mathrm{D}}=C^{\perp} \alpha_{2 \mathrm{D}}^{\perp}
\end{aligned}
$$

where $E_{\mathrm{g}}$ is the fundamental electronic bandgap and $\hat{\delta}_{2 \mathrm{D}}$ is the intrinsic thickness of the $2 \mathrm{D}$ layer, with coefficients $C^{\|}=\frac{N e^{2}}{2 \pi},{ }^{30}$ where $N$ is a pre-factor associated with the band degeneracy, and $C^{\perp}=\varepsilon_{0}{ }^{-1}$. It is worth noting, unlike the parameter $\delta_{2 \mathrm{D}}^{*}$ that is artificially assigned within the EDM picture (see previous section), $\hat{\delta}_{2 \mathrm{D}}$ can be uniquely defined by $\alpha_{2 \mathrm{D}}^{\|}$, a quantity that can be 

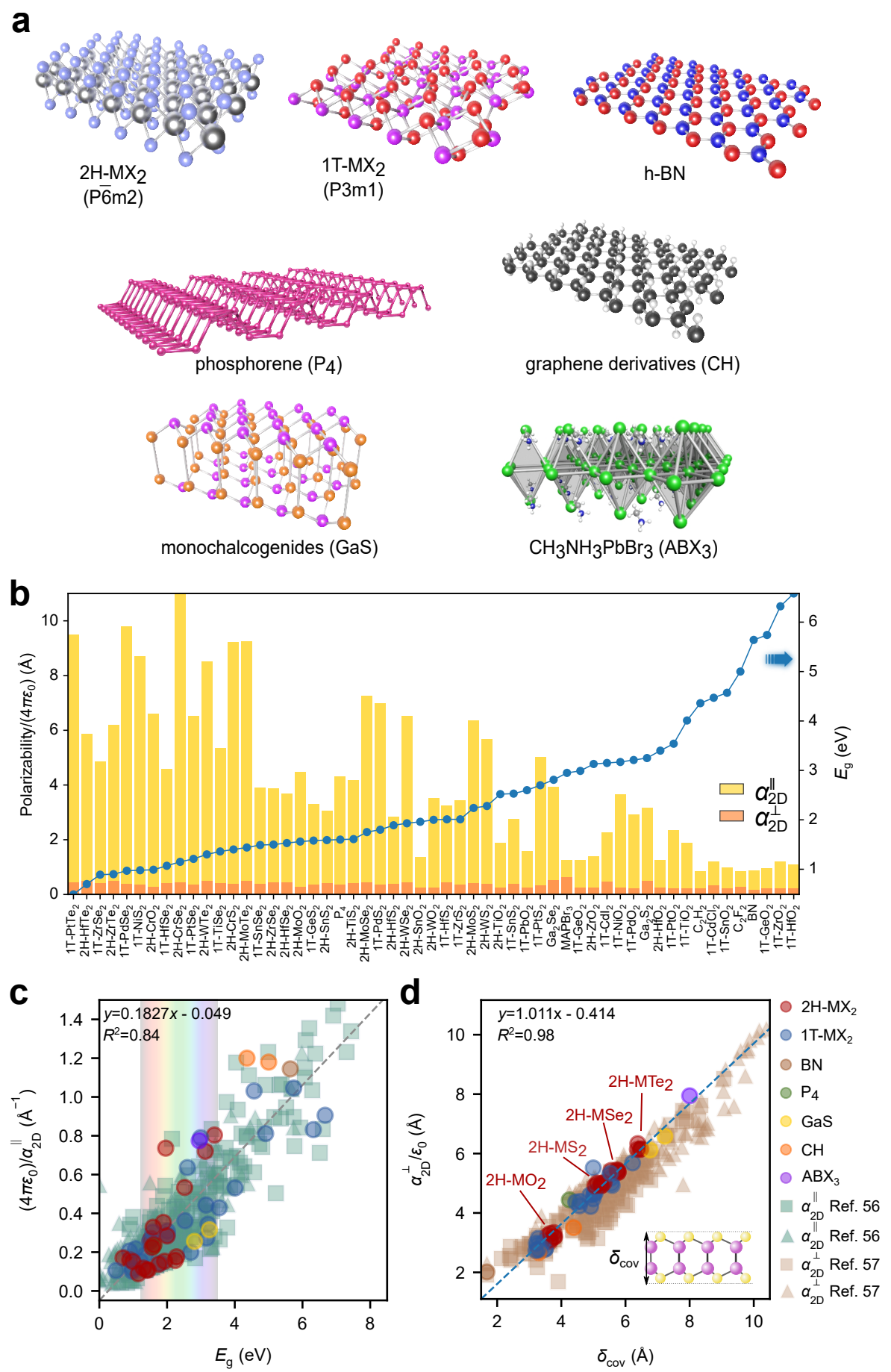

Figure 2: The universal scaling relation of the dielectric nature of $2 \mathrm{D}$ materials. a, The structures of the 2D materials investigated in this study. b, $\alpha_{2 \mathrm{D}}^{\|}, \alpha_{2 \mathrm{D}}^{\perp}$ (bar plots) and $E_{\mathrm{g}}$ (blue dots) for all the $2 \mathrm{D}$ materials studied. $\alpha_{2 \mathrm{D}}^{\|}$is observed to descend with increasing $E_{\mathrm{g}}$, while no apparent relation between $\alpha_{2 \mathrm{D}}^{\perp}$ and $E_{\mathrm{g}}$ is observed. HSE06 functional is used to obtain the data. $\mathbf{c},\left(4 \pi \varepsilon_{0}\right) / \alpha_{2 \mathrm{D}}^{\|}$ (in $\AA^{-1}$ ) as a function of $E_{\mathrm{g}}$, showing a linear correlation between each other. The energy range of visible light is shown in the background. d, $\alpha_{2 \mathrm{D}}^{\perp} /\left(\varepsilon_{0}\right)$ (in $\AA$ ) as a function of $\delta_{\text {cov }}$ (definition schematically shown in the inset), showing a perfect linear relation with a slope very close to 1 (i.e. $\alpha_{2 \mathrm{D}}^{\perp} \approx \varepsilon_{0} \delta_{\text {cov }}$ ). The universal scaling relation is also revealed using different databank from from Ref. 50 (squares), and Ref. 51 (triangles) as superimposed on $\mathbf{c}$ and d. Data corresponding to $2 \mathrm{H}-\mathrm{MX}_{2}(\mathrm{M}=\mathrm{Mo}, \mathrm{W} ; \mathrm{X}=\mathrm{O}, \mathrm{S}, \mathrm{Se}, \mathrm{Te})$ is highlighted in $\mathbf{d}$. The very tiny difference in $\alpha_{2 \mathrm{D}}^{\perp} /\left(\varepsilon_{0}\right)$ between compounds with different metal atoms gives superposed magnitudes not distinguishable in the plot. 
computationally and experimentally determined. Despite the simplicity of Eqs. 4a and 4b, they generate direct relationships between the electronic polarizability and the electronic/structural properties for any $2 \mathrm{D}$ material in a new framework.

Next, we show that these equations are valid for the current library of known layered materials involving different lattice symmetry, element composition, optical and electronic properties (Figure 2a). A high-throughput screening performed on different families of TMDCs $\left(\mathrm{MX}_{2}\right.$, where $\mathrm{M}$ is a metal in groups $4,6,10$, and $\mathrm{X}=\mathrm{O}, \mathrm{S}, \mathrm{Se}, \mathrm{Te})$ and phases ( $\mathrm{P} \overline{\mathrm{m}} 2, \mathrm{P} 3 \mathrm{~m} 1)$, metal monochalcogenides $\left(\mathrm{Ga}_{2} \mathrm{~S}_{2}, \mathrm{Ga}_{2} \mathrm{Se}_{2}\right)$, cadmium halides $(\mathrm{CdX}, \mathrm{X}=\mathrm{Cl}, \mathrm{I})$, hexagonal boron nitride (BN), graphene derivatives (fluorographene $\left(\mathrm{C}_{2} \mathrm{~F}_{2}\right)$, graphane $\left(\mathrm{C}_{2} \mathrm{H}_{2}\right)$ ), phosphorene $\left(\mathrm{P}_{4}\right)$ and thin layer organic-inorganic perovskites $\left(\mathrm{ABX}_{3}\right)$, shows that our method enables full correlation between these disparate variables. Figure $2 \mathbf{b}$ compares the calculated fundamental bandgap $E_{\mathrm{g}}$ (blue dots) and 2D electronic polarizabilities (bar plots) of all the 2D materials investigated, covering a wide spectrum range from far-infrared to ultraviolet. Note that from dimension analysis, it is more intuitive to express the polarizability as $\alpha_{2 \mathrm{D}} /\left(4 \pi \varepsilon_{0}\right)$, which has unit of $\AA$. We find that $\alpha_{2 \mathrm{D}}^{\|}$has a general descending trend when $E_{\mathrm{g}}$ increases, while no apparent correlation between $\alpha_{2 \mathrm{D}}^{\perp}$ and $E_{\mathrm{g}}$ is observed (see Supplementary Section S4). We then examine Eqs. 4a and 4b using the polarizabilities by first-principle calculations. Figure $2 \mathbf{c}$ shows $\left(4 \pi \varepsilon_{0}\right) / \alpha_{2 \mathrm{D}}^{\|}\left(\right.$in $\left.\AA^{-1}\right)$ as a function of $E_{\mathrm{g}}$ (in $\mathrm{eV}$ ) for the 2D materials investigated using HSE06 hybrid functional (circular dots) and PBE (triangles and squares). A linear regression coefficient of $R^{2}=0.84$ indicates a strong correlation between bandgaps and polarizabilities as predicted in Eq. 4a. We also discovered that the linearity between $\left(4 \pi \varepsilon_{0}\right) / \alpha_{2 \mathrm{D}}^{\|}$and $E_{\mathrm{g}}$ (measured by the $R^{2}$ value) is higher when the bandgap is calculated using the HSE06 hybrid functional compared with that from PBE exchange-correlation functional (see Supplementary Section S4 and Supplementary Figure 14). This is reasonable as the bandgaps for 2D materials obtained at the PBE functional, although may be close to experimental reported optical transition energies, are an artifact of the simulation due to a fortuitous error cancellation. ${ }^{52,53}$ Thus, the use of a time-consuming hybrid functional in our study is justified. A detailed benchmark of Eqs. 4a and 4b using different bandgaps, databases, and levels of theory can 
be seen in Supplementary Sections S4-S5.

We further examine the validity of Eq. $4 \mathrm{~b}$, that is, the relation between $\alpha_{2 \mathrm{D}}^{\perp}$ and the thickness of a $2 \mathrm{D}$ material. To test if the quantity $\hat{\delta}_{2 \mathrm{D}}$ is physical, we choose the "covalent" thickness $\delta_{\text {cov }}$ as a comparison. $\delta_{\text {cov }}$ is defined as the longest distance along the $z$-direction between any two atom nuclei plus their covalent radii:

$$
\delta_{\mathrm{cov}}=\max \left(\left|z^{i}-z^{j}\right|+r_{\mathrm{cov}}^{i}+r_{\mathrm{cov}}^{j}\right)
$$

where $i, j$ are atomic indices in the 2D material and $r_{\mathrm{cov}}^{i}$ is the covalent radius of atom $i$ (inset in Figure 2d). As shown in Figure 2d, $\alpha_{2 \mathrm{D}}^{\perp} / \varepsilon_{0}$ (or equivalently, $\hat{\delta}_{2 \mathrm{D}}$ ) is very close to $\delta_{\text {cov }}$ with a good linear correlation of $R^{2}=0.98$. This result indicates a strong relation between $\alpha_{2 \mathrm{D}}^{\perp}$ and the geometry of the 2D layer, which can be approximated by $\delta_{\text {cov }}$. Similar to the molecular polarizability which characterizes the volume of the electron distribution of an isolated molecule, ${ }^{28} \alpha_{2 \mathrm{D}}^{\perp} / \varepsilon_{0}$ is also naturally related to the characteristic thickness of the electron density of a 2D material. Supplementary Section S3.3 shows an explanation of this behavior from fundamental electrostatics and why $\alpha_{2 \mathrm{D}} / \varepsilon_{0}$ is close to $\delta_{\text {cov }}$. The geometric nature of $\alpha_{2 \mathrm{D}}^{\perp}$ leads to several interesting properties. For instance, the points corresponding to $2 \mathrm{H}-\mathrm{TMDCs}$ with same chalcogenide element (i.e. $2 \mathrm{H}$ $\mathrm{MO}_{2}, 2 \mathrm{H}-\mathrm{MS}_{2}, 2 \mathrm{H}-\mathrm{MSe} 2_{2}$ or $2 \mathrm{H}-\mathrm{MTe}_{2}$, where $\mathrm{M}=\mathrm{Mo}, \mathrm{W}$ ) lie very close in Figure $2 \mathbf{b}$ (detailed values see Supplementary Table S2). This can be briefly explained by the fact that the difference between covalent radii of transition metals (e.g. $\sim 8 \mathrm{pm}$ between Mo and $\mathrm{W}$ ) is much smaller than that between group 16 elements (e.g. $\sim 40$ pm between O and S). Our proposed definition of $\hat{\delta}_{2 \mathrm{D}}$ which is based on Eq. $4 \mathrm{~b}$ will provide insights on some long-existing controversies about the experimental thickness of $2 \mathrm{D}$ materials ${ }^{54}$ through a measurable quantity, e.g. $\alpha_{2 \mathrm{D}}^{\perp} \cdot{ }^{55-57}$

To rule out the possibility that our conclusion are limited by the number of materials used at HSE06 level, we further validate Eqs. 4a and 4b using two different 2D-material databases based on different codes. ${ }^{50,51}$ We extracted the dielectric properties of over 300 compounds calculated at the PBE level, and superimpose with our results in Figure 2c and 2d. The high-throughput 
datasets also show linear trends for both $\left(4 \pi \varepsilon_{0}\right) / \alpha_{2 \mathrm{D}}^{\|}$(in $\AA$ ) vs $E_{\mathrm{g}}$ (in eV) $(y=0.190 x-0.0619$, $R^{2}=0.842$ ) and $\alpha_{2 \mathrm{D}}^{\perp}$ vs $\delta_{\text {cov }}$ (both in $\AA, y=0.904 x+0.0551, R^{2}=0.943$ ) relations. We notice that the linear coefficients are similar but not identical to those calculated at the HSE06 level. The discrepancies may be due to several factors resulted from different choice of functionals, such as the underestimation of the bandgap in PBE, and different description of the exchange-correlation potentials. We note that a more accurate estimation of the coefficients should be performed with larger datasets and accurate functionals which requires further work. Nevertheless, the validity of the linear trends observed for $\alpha_{2 \mathrm{D}}^{\|}$and $\alpha_{2 \mathrm{D}}^{\perp}$ is undeniable. We have also searched for additional relations between the $2 \mathrm{D}$ polarizabilities with other physical quantities, including the effective carrier mass, quantum capacitance (density of states) and total atomic polarizabilities with no apparent correlations being found (Supplementary Section S5.3).

\section{Application in multilayer and bulk systems}

The concept of electronic polarizability is not limited to monolayer materials, and can be applied to multilayer and bulk systems as well. For a 2D-material stack composed of $N$ layers, we can define the electronic polarizability $\alpha_{\mathrm{NL}}$ similarly to Eqs. $2 \mathrm{a}-2 \mathrm{~b}$ by replacing $\alpha_{2 \mathrm{D}}^{\|, \perp}$ to $\alpha_{\mathrm{NL}}^{\|, \perp}$. To check whether such assumption is valid, Figure $3 \mathbf{a}$ and $3 \mathbf{b}$ show $\alpha_{\mathrm{NL}}^{\|}$and $\alpha_{\mathrm{NL}}^{\perp}$ as functions of $N$ for several TMDCs in 2H-phase, respectively. Interestingly, we find that in all cases, $\alpha_{\mathrm{NL}}$ exhibits nearly ideal linear relation with $\alpha_{2 \mathrm{D}}$, such that $\alpha_{\mathrm{NL}}^{\|}=N \alpha_{2 \mathrm{D}}^{\|}$and $\alpha_{\mathrm{NL}}^{\perp}=N \alpha_{2 \mathrm{D}}^{\perp}$. Due to the relatively small applied electric field $(0.01 \mathrm{eV} / \AA ̊)$, the interlayer interactions within the stack are negligible. Under such circumstances, $\alpha_{2 \mathrm{D}}$ of individual layers is additive, which leads to the following general relation:

$$
\alpha_{\mathrm{NL}}^{p}=\sum_{i=1}^{N} \alpha_{2 \mathrm{D}, \mathrm{i}}^{p}, \quad p=\| \text { or } \perp
$$

where $\alpha_{2 \mathrm{D}, \mathrm{i}}$ is the electronic polarizability of layer $i$, and $p$ is the direction of the polarization. This relation can be additionally utilized to calculate screening inside 2D heterostructures. ${ }^{58,59}$ 

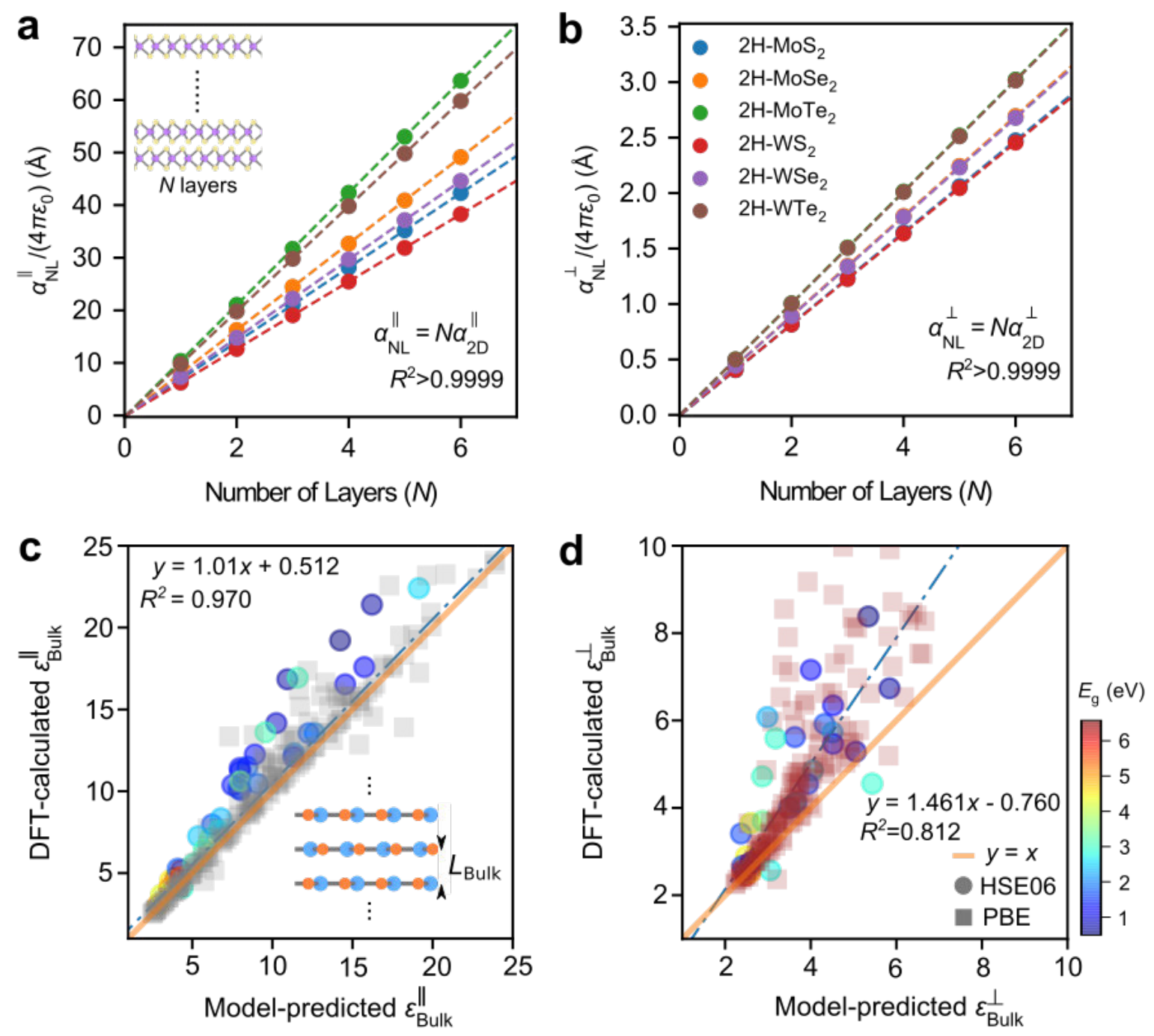

Figure 3: Application of 2D polarizability to few-layer and bulk systems. a-b, Multilayer polarizabilities $\alpha_{\mathrm{NL}}^{\|}$and $\alpha_{\mathrm{NL}}^{\perp}$ of selected 2D metal dichacolgenides $(2 \mathrm{H}-\mathrm{MX}, \mathrm{M}=\mathrm{Mo}, \mathrm{W} ; \mathrm{X}=\mathrm{S}$, $\mathrm{Se}, \mathrm{Te}$ ) as a function of number of layers $N$, respectively. Inset in a, shows a scheme of the 2D-3D transition. $\alpha$ in the $2 \mathrm{D}$ material is essentially equivalent to $\varepsilon$ in its bulk counterpart. Both $\alpha_{\mathrm{NL}}^{\|}$and $\alpha_{\mathrm{NL}}^{\perp}$ linearly scales with $N$ and the electronic polarizability of the monolayer which indicate that $\alpha_{2 \mathrm{D}}$ is an additive quantity under weak interacting regime. c-d, DFT calculated $\varepsilon_{\text {Bulk }}^{\|}$and $\varepsilon_{\text {Bulk }}^{\perp}$, respectively, as a function of their predicted values from the $2 \mathrm{D}$ polarizability model. A strong correlation is observed in both components with the linear regression slope reaching the unit for $\varepsilon_{\text {Bulk }}^{\|}$but slightly deviating for $\varepsilon_{\text {Bulk }}^{\perp}$ at higher magnitudes. A heat map showing the dependence of $\varepsilon_{\text {Bulk }}^{\|, \perp}$ with the band gaps is included in $\mathbf{d}$. The model predicted values for $\varepsilon_{\text {Bulk }}^{\perp}$ are in good aggreement with the DFT calculations when $E_{\mathrm{g}}>4 \mathrm{eV}$. Inset in $\mathbf{c}$, shows the definition of the interlayer distance in bulk $L_{\text {Bulk }}$ utilized to calculate $\varepsilon_{\text {Bulk }}^{\|}$and $\varepsilon_{\text {Bulk }}^{\perp}$ via Eqs. $7 \mathrm{a}-7 \mathrm{a}$. Calculations at the level of HSE06 and PBE are shown in circles and squares, respectively, in all panels that apply. 
In a bulk material with an equilibrium inter-layer distance $L_{\mathrm{Bulk}}$, we can follow a similar pro-

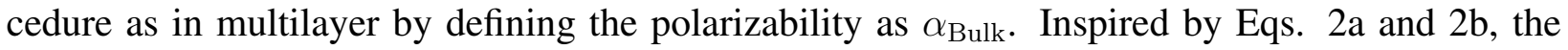
dielectric constants $\varepsilon_{\mathrm{Bulk}}^{\|}$and $\varepsilon_{\mathrm{Bulk}}^{\perp}$ of the bulk layered material can be reconstructed by $\alpha_{\mathrm{Bulk}}^{\|}$and $\alpha_{\mathrm{Bulk}}^{\perp}$ as:

$$
\begin{aligned}
& \varepsilon_{\mathrm{Bulk}}^{\|}=1+\frac{\alpha_{\mathrm{Bulk}}^{\|}}{\varepsilon_{0} L_{\mathrm{Bulk}}} \approx 1+\frac{\alpha_{2 \mathrm{D}}^{\|}}{\varepsilon_{0} L_{\mathrm{Bulk}}} \\
& \varepsilon_{\mathrm{Bulk}}^{\perp}=\left(1-\frac{\alpha_{\mathrm{Bulk}}^{\perp}}{\varepsilon_{0} L_{\mathrm{Bulk}}}\right)^{-1} \approx\left(1-\frac{\alpha_{2 \mathrm{D}}^{\perp}}{\varepsilon_{0} L_{\mathrm{Bulk}}}\right)^{-1}
\end{aligned}
$$

Here we neglect the effect of the stacking order of the layers and hypothesized that the basic building blocks for the dielectric response of the bulk are the polarizability of the individual layers subject to vdW and electrostatic interactions. The dielectric constant $\varepsilon$ although not well-defined for a monolayer 2D material becomes applicable when the 2D layers are put together as shown in the following. We compare the values of $\varepsilon_{\text {bulk }}^{\|}$and $\varepsilon_{\text {bulk }}^{\perp}$ computed from DFT simulations $(y$ axis) with those predicted using Eqs $7 \mathrm{a}$ and $7 \mathrm{~b}$ ( $x$-axis) as shown in Figure $3 \mathbf{c}$ and $3 \mathbf{d}$. Strikingly both HSE06 and PBE datasets give almost identical results which suggest a non-method dependent behavior. We observe that $\varepsilon_{\text {bulk }}^{\|}$values calculated by DFT and predicted by Eq. 7a are in sound agreement with a linear regression slope of 1.01 and $R^{2}$ of 0.97 . Conversely, $\varepsilon_{\text {bulk }}^{\perp}$ values predicted from Eq. 7b fairly agree with the DFT-calculated values when $E_{\mathrm{g}}>4 \mathrm{eV}$, while the deviation becomes larger when $E_{\mathrm{g}}$ reduces. The above results indicate that $\alpha_{\mathrm{Bulk}}^{\|}$can generally be estimated with high accuracy from its 2D counterpart, while $\alpha_{\mathrm{Bulk}}^{\perp}$ differs due to the interlayer coupling and overlap between induced dipole. ${ }^{45,59}$ Nevertheless, as most of the optical response and electronic device properties rely on the in-plane dielectric constant for practical applications, the possibility to handily estimate $\alpha_{2 \mathrm{D}}^{\|}$from well established magnitudes of $\varepsilon_{\text {bulk }}^{\|}$, for instance, from material databases, using reverse engineering in Eq.7a, it is a step forward in the design and understanding of the dielectric phenomena in 2D. 


\section{Unified geometric representation of $\alpha_{2 \mathrm{D}}$}

Lastly, we demonstrate that both $\alpha_{2 \mathrm{D}}^{\|}$and $\alpha_{2 \mathrm{D}}^{\perp}$ can be unified using a geometric approach. In merit of the unit analysis, $\alpha_{2 \mathrm{D}}^{\|}$and $\alpha_{2 \mathrm{D}}^{\perp}$ both have unit of $4 \pi \varepsilon_{0} \times$ [Length]. In other words, they represent in- and out-of-plane characteristic lengths, respectively. It is well-known that the in-plane screened electrostatic potential $V(r)=\frac{e}{4 \alpha_{2 \mathrm{D}}^{\|}}\left[H_{0}\left(\frac{2 \varepsilon_{0} r}{\alpha_{2 \mathrm{D}}^{\|}}\right)-Y_{0}\left(\frac{2 \varepsilon_{0} r}{\alpha_{2 \mathrm{D}}^{\|}}\right)\right]$from a point charge as a function of distance $r^{9,60}$ (where $H_{0}$ is the Struve function and $Y_{0}$ is the Bessel function of second kind) is associated with the in-plane screening radius $r_{0}^{\|}=\alpha_{2 \mathrm{D}}^{\|} /\left(2 \varepsilon_{0}\right)$, such that $V\left(r, r / r_{0}^{\|} \gg 1\right)$ reduces to the simple Coulomb potential in vacuum. Combining with the result that $\alpha_{2 \mathrm{D}}^{\perp} / \varepsilon_{0}$ characterizes the thickness of a 2D material, we can view the dielectric screening of a point charge sitting in the middle of a 2D material as an ellipsoid with the radii of principal axes to be $r_{0}^{\|}=\alpha_{2 \mathrm{D}}^{\|} /\left(2 \varepsilon_{0}\right)$ and $r_{0}^{\perp}=\alpha_{2 \mathrm{D}}^{\perp} /\left(2 \varepsilon_{0}\right)$, respectively, as illustrated in Figure 4a. This is analog to the polarizability ellipsoid picture of molecules used in spectroscopy. ${ }^{61}$ The polarizability ellipsoid for a $2 \mathrm{D}$ material is in general ultra flat, with $r_{0}^{\|} \gg r_{0}^{\perp}$, as demonstrated by layered materials of group 6 of $2 \mathrm{H}-$ TMDCs (Figure $4 \mathbf{b}$ and $4 \mathbf{c}$ ). The picture of the polarizability ellipsoid provides further insights into the physical nature of $\alpha_{2 \mathrm{D}}: r_{0}^{\|}$is close to the exciton radius that it is confined within the 2D plane. ${ }^{60}$ This radius is generally larger for a smaller bandgap semiconductor, and can be converted through the exciton binding energy as proposed in Refs. ${ }^{29,30} r_{0}^{\perp}$ in its turn can be indirectly deduced from Stark effect for perpendicular electric fields. ${ }^{62-64}$ A comparison with available experimental data $^{64,65}$ gives close magnitudes with our predicted values.

Inspired by the polarizability ellipsoid model, we will show that a general picture of the dielectric properties in any dimension can be drawn by studying the dielectric anisotropy. That is, the dielectric response of a material along its different geometrical orientations. We define the dielectric anisotropy index $\eta$ as:

$$
\eta=\left\{\begin{array}{l}
\min _{i \neq j}\left(\frac{\varepsilon^{i i}}{\varepsilon^{j j}}\right), \text { Bulk Materials } \\
\min _{i \neq j}\left(\frac{\alpha_{2 \mathrm{D}}^{i i}}{\alpha_{2 \mathrm{D}}^{j j}}\right), 2 \mathrm{D} \text { Materials }
\end{array}\right.
$$



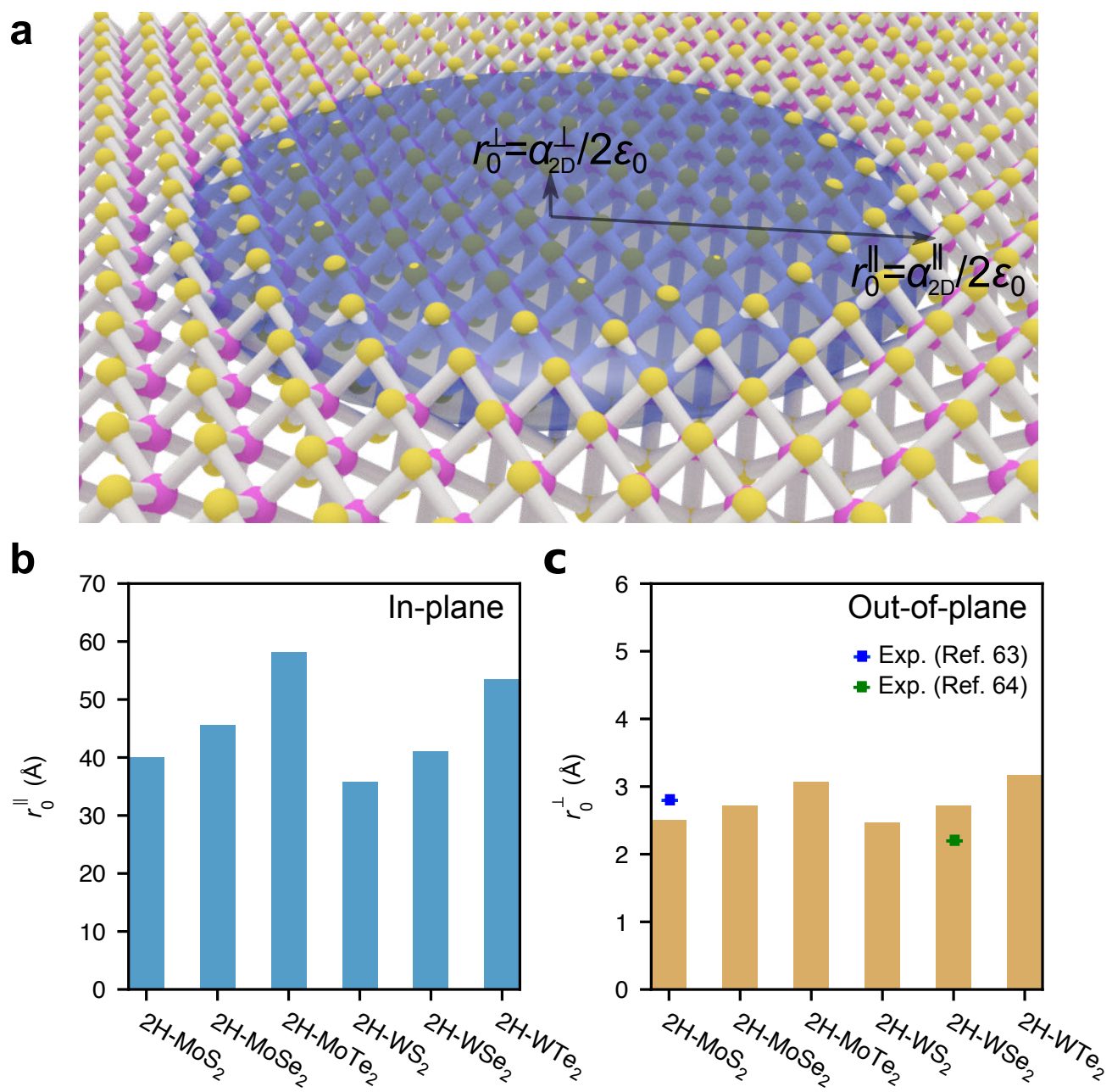

Figure 4: Geometric representation of the 2D polarizability. a, Scheme of the polarizability ellipsoid of a 2D material, with its in-plane $\left(r_{0}^{\|}\right)$and out-of-plane radii $\left(r_{0}^{\perp}\right)$ proportional to $\alpha_{2 \mathrm{D}}^{\|}$and $\alpha_{2 \mathrm{D}}^{\perp}$, respectively. b-c, Calculated magnitudes of $r_{0}^{\|}$and $r_{0}^{\perp}$, respectively, for selected 2D TMDCs. The polarizability ellipsoid is highly anisotropic with screening much stronger at in-plane than out-of-plane directions. Comparison with available experimental results ${ }^{64,65}$ for $2 \mathrm{H}-\mathrm{MoS}_{2}$ and $2 \mathrm{H}-\mathrm{WSe} \mathrm{H}_{2}$ is included.

$\eta=1$ indicates that the material has isotropic dielectric properties while $\eta \rightarrow 0$ means that the dielectric property is highly anisotropic. Figure 5 shows the phase diagram of $\eta$ as function of $E_{\mathrm{g}}$ for 2D materials and their bulk counterparts. Interestingly, the 2D materials (blue triangles) can be clearly distinguished from the bulk layered materials (orange squares) with the boundary line determined to be $\eta=0.048\left(E_{\mathrm{g}} / \mathrm{eV}\right)+0.087$. The much lower $\eta$ values for $2 \mathrm{D}$ materials compared with their bulk counterparts indicates a high dielectric anisotropy, which is responsible for the unique $2 \mathrm{D}$ optoelectronic properties, such as the electrostatic transparency phenomena ${ }^{66-68}$ 
and the large exciton binding energies. ${ }^{60,69-71}$ From Eqs. $4 \mathrm{a}, 4 \mathrm{~b}$ and 8 we can see $\eta$ is roughly proportional to $E_{\mathrm{g}} \times \delta$, which explains the observation that $\eta$ for $2 \mathrm{D}$ materials increase almost linearly with $E_{\mathrm{g}}$, since the layer thickness $\delta$ (mostly 3-10 $\AA$ ) of the 2D materials investigated varies much less than $E_{\mathrm{g}}$ in the range of $0.1-7 \mathrm{eV}$ (Figure $2 \mathrm{~b}-2 \mathrm{c}$ ). Further analysis shows that the dielectric anisotropy index of any bulk layered material $\eta_{\mathrm{Bulk}}$ obeys $\eta_{\mathrm{Bulk}} \geq \frac{4 \eta_{2 \mathrm{D}}}{\left(\eta_{2 \mathrm{D}}+1\right)^{2}} \geq \eta_{2 \mathrm{D}}$, where $\eta_{2 \mathrm{D}}$ is the anisotropy index of corresponding $2 \mathrm{D}$ layer, which is the basis for the separation of bulk and 2D regimes in the $\eta-E_{\mathrm{g}}$ phase diagram (Supplementary Section S7). For comparison, we also superimpose the dielectric anisotropy indices of common semiconducting materials in other dimensions on the phase diagram in Figure 5. Bulk covalent 3D (e.g. Si, GaN) and 0D (e.g. fullerenes) semiconductors show isotropic dielectric properties, scattered along the line $\eta=1$. Conversely, reduced dimensionality increases the dielectric anisotropy of materials such as planar organic semiconductor $(\mathrm{OSc})$ in $1 \mathrm{D}-2 \mathrm{D}$ (e.g. CuPc), carbon nanotube $(\mathrm{CNT})$ in $1 \mathrm{D}$, linear OSc in 0D-1D (e.g. polyacene and polyacetylene). Interestingly, most of these materials also scatter along the boundary line separating the bulk and 2D regimes, indicating that the criteria distinguishing $2 \mathrm{D}$ (more anisotropic) and bulk materials (more isotropic) from the $\eta-E_{\mathrm{g}}$ diagram, can also be applied to other dimensions. From the phase diagram, we can see that $2 \mathrm{D}$ and bulk layered materials, including 2D van der Waals heterostructure $(\mathrm{vdWH}),{ }^{8}$ provides more flexibility in controlling the dielectric and electronic properties, compared with covalent semiconductors (without vdW gaps) in other dimensions. 

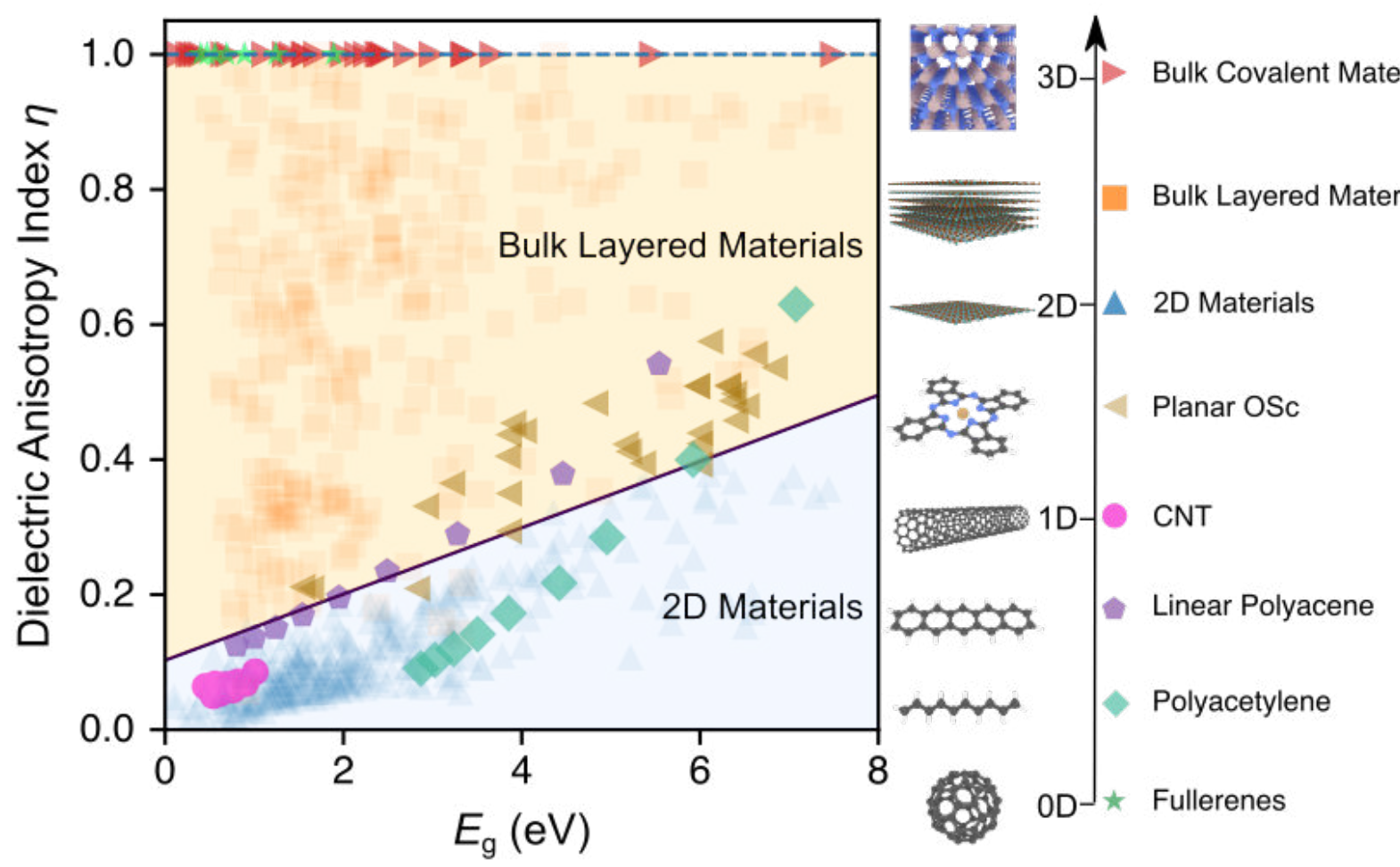

Figure 5: Phase diagram of dielectric anisotropy $\eta$ as a function of bandgap $E_{\mathrm{g}}$. The $\eta-E_{\mathrm{g}}$ values of 2D materials (blue triangle) and their bulk counterparts (orange square) can be distinguished by the line $\eta=0.048\left(E_{\mathrm{g}} / \mathrm{eV}\right)+0.087 . \eta-E_{\mathrm{g}}$ values of semiconducting materials in other dimensions are also superimposed for comparison. Isotropic dielectric property is observed for bulk covalent materials (3D, red triangle) and fullerenes ( $0 \mathrm{D}$, green star), while reduced dimensional materials, including planar organic semiconductor(OSc, 1D-2D, brown triangle), carbon nanotube (CNT, magenta circle) and linear OSc (OD-1D, violet pentagon) are scattered along the boundary line. The dimensionality and structure of typical materials are shown along the axis on the right. Compared with other materials, 2D materials and their bulk counterparts provide more flexibility of controlling the dielectric anisotropy.

\section{Conclusion}

Our results show that the $2 \mathrm{D}$ electronic polarizability $\alpha_{2 \mathrm{D}}$ is a local variable determining the dielectric properties of 2D materials. There exist well-defined relationships between $\alpha_{2 \mathrm{D}}$ and other quantities hidden in the electronic properties. According to our analysis, simple scaling equations involving bandgap and layer thickness can be used to describe both dielectric and electronic features at the same footing. A dielectric anisotropy index is found relating any material dimension 
with its controllability. Thus, our results suggest that the challenge of understanding the dielectric phenomena is in general a geometrical problem mediated by the bandgap. We believe the principles presented here will benefit both fundamental understanding of 2D materials as well as a rational device design and optimization.

\section{Theoretical Methods}

Simulations were carried out using plane-wave density functional theory package VASP ${ }^{72-74}$ using the projector augmented wave (PAW) approach with GW pseudopotentials. ${ }^{75}$ Band gaps were calculated using the Heyd-Scuseria-Ernzerhof hybrid functional (HSE06), ${ }^{20,21}$ with spin orbit coupling (SOC) explicitly included. The geometries were converged both in cell parameters and ionic positions, with forces below $0.04 \mathrm{eV} / \AA \AA$. To ensure the accuracy of dielectric property of monolayer, a vacuum spacing of $>15 \AA$ is used. A k-point grid of $7 \times 7 \times 1$ was used to relax the superlattice, with an initial relaxation carried out at the Perdew-Burke-Ernzerhof (PBE) ${ }^{22,24 ?}$ exchangecorrelation functional level and a subsequent relaxation carried out at HSE06 level, allowing both cell parameters and ionic positions to relax each time. In VASP, the tag PREC=High was used, giving a plane wave kinetic energy cutoff of $30 \%$ greater than the highest given in the pseudopotentials used in each material. This guarantees that absolute energies were converged to a few meV and the stress tensor to within $0.01 \mathrm{kBar}$. Calculation of the macroscopic ion-clamped dielectric tensor were carried out with an $18 \times 18 \times 1 \mathrm{k}$-grid and electric field strength of $0.001 \mathrm{eV} / \AA$. Local field effect corrections are included at the exchange-correlation potential $V_{\mathrm{xc}}$ at both PBE and HSE06 levels. The materials from Ref. 50 for comparison were choses with the GW bandgap larger than $0.05 \mathrm{eV}$. Bulk layered materials were constructed by relaxing the c-axis length of corresponding monolayer material with the interlayer van der Waals (vdW) interactions calculated by non-local $\mathrm{vdW}$ correlation functional. ${ }^{76}$ The dielectric properties of bulk layered materials using VASP were calculated at HSE06 level with $18 \times 18 \times 6 \mathrm{k}$-grid with same parameter as for monolayer, while the dielectric properties of bulk counterparts of Ref. 50 are calculated at PBE level with a k-point 
density of $10 \AA^{-1}$. Local field effect corrections are also used for the dielectric properties of bulk systems.

\section{Data Availability}

The data that support the findings of this study is available within the paper and its Supplementary Information.

\section{Competing interests}

The Authors declare no conflict of interests.

\section{Acknowledgments}

C.J.S. and T.T. are grateful for financial support from ETH startup funding. L.H.L. thanks the financial support from Australian Research Council (ARC) via Discovery Early Career Researcher Award (DE160100796). E.J.G.S. acknowledges the use of computational resources from the UK Materials and Molecular Modelling Hub for access to THOMAS supercluster, which is partially funded by EPSRC (EP/P020194/1); and CIRRUS Tier-2 HPC Service (ec019 Cirrus Project) at EPCC (http://www.cirrus.ac.uk) funded by the University of Edinburgh and EPSRC (EP/P020267/1). The Department for the Economy (USI 097) is acknowledged for funding support.

\section{Author Contributions}

E.J.G.S. conceived the idea and supervised the project. T.T., D.S., D.H. and E.J.G.S. performed the first-principles simulations and data analytics. T.T. developed the analytical model with inputs from E.J.G.S. and C.J.S. L.H.L. and J.N.C. performed numerical analysis and contributed to the discussions together with M.C. E.J.G.S. and T.T. co-wrote the manuscript with inputs from all authors. All authors contributed to this work, read the manuscript, discussed the results, and all agree to the contents of the manuscript. 


\section{Supporting Information}

The Supporting Information contains detailed descriptions and discussions about dielectric properties of 2D materials, effective dielectric model, derivations of the 2D polarizability-based model, dependency of $\alpha_{2 \mathrm{D}}$ on the choice of bandgap, relation between 2D and 3D properties, explanations of the dielectric anisotropy, as well as raw data sheet from first principles calculations.

\section{References}

1. Moss, T. S. A Relationship between the Refractive Index and the Infra-Red Threshold of Sensitivity for Photoconductors. Proc. Phys. Soc. B 1950, 63, 167.

2. Moss, T. S. Relations between the Refractive Index and Energy Gap of Semiconductors. Phys. Status Solidi B 1985, 131, 415-427.

3. Kittel, C. Introduction to solid state physics; Wiley, 2005.

4. Dressel, M.; Gruner, G. Electrodynamics of solids: optical properties of electrons in matter; Cambridge University Press, 2001.

5. Adler, S. L. Quantum Theory of the Dielectric Constant in Real Solids. Phys. Rev. 1962, 126, 413-420.

6. Hybertsen, M. S.; Louie, S. G. Ab initiostatic dielectric matrices from the density-functional approach. I. Formulation and application to semiconductors and insulators. Phys. Rev. B 1987, $35,5585-5601$.

7. Palik, E. D., Ed. Handbook of optical constants of solids; Academic Press, 1998.

8. Novoselov, K. S.; Mishchenko, A.; Carvalho, A.; Castro Neto, A. H. 2D materials and van der Waals heterostructures. Science 2016, 353, aac9439. 
9. Keldysh, L. V. Coulomb interaction in thin semiconductor and semimetal films. Sov. Phys. JETP 1979, 29, 658.

10. Sharma, A. Dielectric function of a semiconductor slab. J. Phys. C: Solid State Phys. 1985, 18, L153-L156.

11. Low, T.; Roldán, R.; Wang, H.; Xia, F.; Avouris, P.; Moreno, L. M.; Guinea, F. Plasmons and Screening in Monolayer and Multilayer Black Phosphorus. Phys. Rev. Lett. 2014, 113, 106802.

12. Cudazzo, P.; Tokatly, I. V.; Rubio, A. Dielectric screening in two-dimensional insulators: Implications for excitonic and impurity states in graphane. Phys. Rev. B 2011, 84, 085406.

13. Bechstedt, F.; Matthes, L.; Gori, P.; Pulci, O. Infrared absorbance of silicene and germanene. Appl. Phys. Lett. 2012, 100, 261906.

14. Cudazzo, P.; Attaccalite, C.; Tokatly, I. V.; Rubio, A. Strong Charge-Transfer Excitonic Effects and the Bose-Einstein Exciton Condensate in Graphane. Phys. Rev. Lett. 2010, 104, 226804.

15. Nazarov, V. U. Electronic excitations in quasi-2D crystals: what theoretical quantities are relevant to experiment? New J. Phys. 2015, 17, 073018.

16. Li, S.-L.; Tsukagoshi, K.; Orgiu, E.; Samorì, P. Charge transport and mobility engineering in two-dimensional transition metal chalcogenide semiconductors. Chem. Soc. Rev. 2016, 45, $118-151$.

17. Sohier, T.; Gibertini, M.; Calandra, M.; Mauri, F.; Marzari, N. Breakdown of Optical Phonons’ Splitting in Two-Dimensional Materials. Nano Lett. 2017, 17, 3758-3763.

18. Laturia, A.; Vandenberghe, W. G. Dielectric properties of mono- and bilayers determined from first principles. 2017 International Conference on Simulation of Semiconductor Processes and Devices (SISPAD). 2017; pp 337-340. 
19. Sohier, T.; Calandra, M.; Mauri, F. Two-dimensional Fröhlich interaction in transition-metal dichalcogenide monolayers: Theoretical modeling and first-principles calculations. Phys. Rev. B 2016, 94, 085415.

20. Heyd, J.; Scuseria, G. E.; Ernzerhof, M. Hybrid functionals based on a screened Coulomb potential. J. Chem. Phys. 2003, 118, 8207-8215.

21. Heyd, J.; Scuseria, G. E.; Ernzerhof, M. Erratum: "Hybrid functionals based on a screened Coulomb potential" [J. Chem. Phys. 118, 8207 (2003)]. J. Chem. Phys. 2006, 124, 219906.

22. Perdew, J. P.; Burke, K.; Ernzerhof, M. Generalized Gradient Approximation Made Simple. Phys. Rev. Lett. 1996, 77, 3865-3868.

23. Ernzerhof, M.; Scuseria, G. E. Assessment of the Perdew-Burke-Ernzerhof exchangecorrelation functional. J. Chem. Phys. 1999, 110, 5029-5036.

24. Paier, J.; Hirschl, R.; Marsman, M.; Kresse, G. The Perdew-Burke-Ernzerhof exchangecorrelation functional applied to the G2-1 test set using a plane-wave basis set. J. Chem. Phys. 2005, 122, 234102 .

25. Hedin, L. New Method for Calculating the One-Particle Green's Function with Application to the Electron-Gas Problem. Phys. Rev. 1965, 139, A796-A823.

26. Onida, G.; Reining, L.; Rubio, A. Electronic excitations: density-functional versus many-body Green's-function approaches. Rev. Mod. Phys. 2002, 74, 601-659.

27. Matthes, L.; Pulci, O.; Bechstedt, F. Influence of out-of-plane response on optical properties of two-dimensional materials: First principles approach. Phys. Rev. B 2016, 94, 205408.

28. Israelachvili, J. N. Interactions Involving the Polarization of Molecules. Intermolecular and Surface Forces 2011, 91-106.

29. Olsen, T.; Latini, S.; Rasmussen, F.; Thygesen, K. S. Simple Screened Hydrogen Model of Excitons in Two-Dimensional Materials. Phys. Rev. Lett. 2016, 116, 056401. 
30. Jiang, Z.; Liu, Z.; Li, Y.; Duan, W. Scaling Universality between Band Gap and Exciton Binding Energy of Two-Dimensional Semiconductors. Phys. Rev. Lett. 2017, 118.

31. Tobik, J.; Dal Corso, A. Electric fields with ultrasoft pseudo-potentials: Applications to benzene and anthracene. J. Chem. Phys. 2004, 120, 9934-9941.

32. Wiser, N. Dielectric Constant with Local Field Effects Included. Phys. Rev. 1963, 129, 62-69.

33. Benedict, L. X.; Louie, S. G.; Cohen, M. L. Static polarizabilities of single-wall carbon nanotubes. Phys. Rev. B 1995, 52, 8541-8549.

34. Markel, V. A. Introduction to the Maxwell Garnett approximation: tutorial. J. Opt. Soc. Am. A 2016, 33, 1244.

35. Meyer, B.; Vanderbilt, D. Ab initiostudy of $\mathrm{BaTiO}_{3}$ and $\mathrm{PbTiO}_{3}$ surfaces in external electric fields. Phys. Rev. B 2001, 63, 205426.

36. Wurstbauer, U.; Röling, C.; Wurstbauer, U.; Wegscheider, W.; Vaupel, M.; Thiesen, P. H.; Weiss, D. Imaging ellipsometry of graphene. Appl. Phys. Lett. 2010, 97, 231901.

37. Yim, C.; O’Brien, M.; McEvoy, N.; Winters, S.; Mirza, I.; Lunney, J. G.; Duesberg, G. S. Investigation of the optical properties of $\mathrm{MoS}_{2}$ thin films using spectroscopic ellipsometry. Appl. Phys. Lett. 2014, 104, 103114.

38. Shen, C.-C.; Hsu, Y.-T.; Li, L.-J.; Liu, H.-L. Charge Dynamics and Electronic Structures of Monolayer MoS 2 Films Grown by Chemical Vapor Deposition. Appl. Phys. Express 2013, 6, 125801.

39. Li, W.; Birdwell, A. G.; Amani, M.; Burke, R. A.; Ling, X.; Lee, Y.-H.; Liang, X.; Peng, L.; Richter, C. A.; Kong, J. et al. Broadband optical properties of large-area monolayer CVD molybdenum disulfide. Phys. Rev. B 2014, 90, 195434. 
40. Li, Y.; Chernikov, A.; Zhang, X.; Rigosi, A.; Hill, H. M.; van der Zande, A. M.; Chenet, D. A.; Shih, E.-M.; Hone, J.; Heinz, T. F. Measurement of the optical dielectric function of monolayer transition-metal dichalcogenides: $\mathrm{MoS}_{2}, \mathrm{MoSe}_{2}, \mathrm{WS}_{2}$, andWSe 2 . Phys. Rev. B 2014, 90, 205422.

41. Wilson, J.; Yoffe, A. The transition metal dichalcogenides discussion and interpretation of the observed optical, electrical and structural properties. Adv. Phys. 1969, 18, 193-335.

42. Meckbach, L.; Stroucken, T.; Koch, S. W. Influence of the effective layer thickness on the ground-state and excitonic properties of transition-metal dichalcogenide systems. Phys. Rev. B 2018, 97, 035425 .

43. Trolle, M. L.; Pedersen, T. G.; Véniard, V. Model dielectric function for 2D semiconductors including substrate screening. Sci. Rep. 2017, 7, 39844.

44. Aspnes, D. Optical properties of thin films. Thin Solid Films 1982, 89, 249-262.

45. Laturia, A.; Van de Put, M. L.; Vandenberghe, W. G. Dielectric properties of hexagonal boron nitride and transition metal dichalcogenides: from monolayer to bulk. npj 2D Mater. Appl. 2018, 2,6 .

46. Tancogne-Dejean, N.; Giorgetti, C.; Véniard, V. Optical properties of surfaces with supercellab initiocalculations: Local-field effects. Phys. Rev. B 2015, 92, 245308.

47. Finkenrath, $\mathrm{H}$. The moss rule and the influence of doping on the optical dielectric constant of semiconductors-II. Infrared Physics 1988, 28, 363-366.

48. Ravindra, N. M.; Srivastava, V. K. Electronic polarizability as a function of the penn gap in semiconductors. Infrared Phys. 1980, 20, 67-69.

49. Ravindra, N.; Srivastava, V. Variation of refractive index with energy gap in semiconductors. Infrared Phys. 1979, 19, 603-604. 
50. Haastrup, S.; Strange, M.; Pandey, M.; Deilmann, T.; Schmidt, P. S.; Hinsche, N. F.; Gjerding, M. N.; Torelli, D.; Larsen, P. M.; Riis-Jensen, A. C. et al. The Computational 2D Materials Database: high-throughput modeling and discovery of atomically thin crystals. $2 D$ Mater. 2018, 5, 042002.

51. Mounet, N.; Gibertini, M.; Schwaller, P.; Campi, D.; Merkys, A.; Marrazzo, A.; Sohier, T.; Castelli, I. E.; Cepellotti, A.; Pizzi, G. et al. Two-dimensional materials from high-throughput computational exfoliation of experimentally known compounds. Nat. Nanotechnol. 2018, 13, $246-252$.

52. Kuc, A.; Heine, T. The electronic structure calculations of two-dimensional transition-metal dichalcogenides in the presence of external electric and magnetic fields. Chem. Soc. Rev. 2015, $44,2603-2614$.

53. Lee, J.; Huang, J.; Sumpter, B. G.; Yoon, M. Strain-engineered optoelectronic properties of 2D transition metal dichalcogenide lateral heterostructures. 2D Materials 2017, 4, 021016.

54. Shearer, C. J.; Slattery, A. D.; Stapleton, A. J.; Shapter, J. G.; Gibson, C. T. Accurate thickness measurement of graphene. Nanotechnology 2016, 27, 125704.

55. Antoine, R.; Dugourd, P.; Rayane, D.; Benichou, E.; Broyer, M.; Chandezon, F.; Guet, C. Direct measurement of the electric polarizability of isolated C60 molecules. J. Chem. Phys. 1999, 110, 9771-9772.

56. Cherniavskaya, O.; Chen, L.; Weng, V.; Yuditsky, L.; Brus, L. E. Quantitative Noncontact Electrostatic Force Imaging of Nanocrystal Polarizability. J. Phys. Chem. B 2003, 107, 15251531.

57. Krauss, T. D.; Brus, L. E. Charge, Polarizability, and Photoionization of Single Semiconductor Nanocrystals. Phys. Rev. Lett. 1999, 83, 4840-4843. 
58. Kumar, P.; Chauhan, Y. S.; Agarwal, A.; Bhowmick, S. Thickness and Stacking Dependent Polarizability and Dielectric Constant of Graphene-Hexagonal Boron Nitride Composite Stacks. J. Phys. Chem. C 2016, 120, 17620-17626.

59. Andersen, K.; Latini, S.; Thygesen, K. S. Dielectric Genome of van der Waals Heterostructures. Nano Lett. 2015, 15, 4616-4621.

60. Pulci, O.; Marsili, M.; Garbuio, V.; Gori, P.; Kupchak, I.; Bechstedt, F. Excitons in twodimensional sheets with honeycomb symmetry. Phys. Status Solidi B 2014, 252, 72-77.

61. Banwell, C. N.; McCash, E. M. Fundamentals of molecular spectroscopy; McGraw-Hill New York, 1994; Vol. 851.

62. Pedersen, T. G. Exciton Stark shift and electroabsorption in monolayer transition-metal dichalcogenides. Phys. Rev. B 2016, 94.

63. Klein, J.; Wierzbowski, J.; Regler, A.; Becker, J.; Heimbach, F.; Müller, K.; Kaniber, M.; Finley, J. J. Stark Effect Spectroscopy of Mono- and Few-Layer $\mathrm{MoS}_{2}$. Nano Lett. 2016, 16, $1554-1559$.

64. Roch, J. G.; Leisgang, N.; Froehlicher, G.; Makk, P.; Watanabe, K.; Taniguchi, T.; Schönenberger, C.; Warburton, R. J. Quantum-Confined Stark Effect in a $\mathrm{MoS}_{2}$ Monolayer van der Waals Heterostructure. Nano Lett. 2018, 18, 1070-1074.

65. Verzhbitskiy, I.; Vella, D.; Watanabe, K.; Taniguchi, T.; Eda, G. Suppressed Out-of-Plane Polarizability of Free Excitons in Monolayer WSe ${ }_{2}$. ACS Nano 2019, 13, 3218-3224.

66. Li, L. H.; Santos, E. J. G.; Xing, T.; Cappelluti, E.; Roldán, R.; Chen, Y.; Watanabe, K.; Taniguchi, T. Dielectric Screening in Atomically Thin Boron Nitride Nanosheets. Nano Lett. 2014, 15, 218-223.

67. Tian, T.; Rice, P.; Santos, E. J. G.; Shih, C.-J. Multiscale Analysis for Field-Effect Penetration through Two-Dimensional Materials. Nano Lett. 2016, 16, 5044-5052. 
68. Li, L. H.; Tian, T.; Cai, Q.; Shih, C.-J.; Santos, E. J. G. Asymmetric electric field screening in van der Waals heterostructures. Nat. Commun. 2018, 9, 1271.

69. Tran, V.; Soklaski, R.; Liang, Y.; Yang, L. Layer-controlled band gap and anisotropic excitons in few-layer black phosphorus. Phys. Rev. B 2014, 89, 235319.

70. Chernikov, A.; Berkelbach, T. C.; Hill, H. M.; Rigosi, A.; Li, Y.; Aslan, O. B.; Reichman, D. R.; Hybertsen, M. S.; Heinz, T. F. Exciton Binding Energy and Nonhydrogenic Rydberg Series in Monolayer $\mathrm{WS}_{2}$. Phys. Rev. Lett. 2014, 113, 076802.

71. Berkelbach, T. C.; Hybertsen, M. S.; Reichman, D. R. Theory of neutral and charged excitons in monolayer transition metal dichalcogenides. Phys. Rev. B 2013, 88, 045318.

72. Kresse, G.; Hafner, J. Ab initiomolecular dynamics for liquid metals. Phys. Rev. B 1993, 47, $558-561$.

73. Kresse, G.; Furthmüller, J. Efficiency of ab-initio total energy calculations for metals and semiconductors using a plane-wave basis set. Comput. Mater. Sci. 1996, 6, 15-50.

74. Kresse, G.; Furthmüller, J. Efficient iterative schemes forab initiototal-energy calculations using a plane-wave basis set. Phys. Rev. B 1996, 54, 11169-11186.

75. Kresse, G.; Joubert, D. From ultrasoft pseudopotentials to the projector augmented-wave method. Phys. Rev. B 1999, 59, 1758-1775.

76. Lee, K.; Murray, E. D.; Kong, L.; Lundqvist, B. I.; Langreth, D. C. Higher-accuracy van der Waals density functional. Phys. Rev. B 2010, 82, 081101(R). 


\section{Supplementary Information:}

\section{Electronic polarizability as the fundamental variable in the dielectric properties of two-dimensional materials}

Tian Tian, ${ }^{\dagger, \perp}$ Declan Scullion,,$+\perp$ Dale Hughes, ${ }^{\ddagger}$ Lu Hua Li, ${ }^{\llbracket}$ Chih-Jen Shih, ${ }^{\dagger}$ Jonathan Coleman, ${ }^{\S}$ Manish Chhowalla,$\|$ and Elton J. G. Santos ${ }^{*, \ddagger}$

$\dagger$ Institute for Chemical and Bioengineering, ETH Zürich, Vladimir Prelog Weg 1, CH-8093 Zürich, Switzerland

$\ddagger$ School of Mathematics and Physics, Queen’s University Belfast, BT7 1NN, United Kingdom ФInstitute for Frontier Materials, Deakin University, Victoria, VIC 3216, Australia $\S$ School of Physics, Centre for Research on Adaptive Nanostructures and Nanodevices (CRANN) and Advanced Materials and BioEngineering Research (AMBER), Trinity College Dublin, Dublin 2, Ireland.

||Department of Materials Science \& Metallurgy, University of Cambridge, CB3 OFS, United Kingdom

$\perp$ T. T. and D. S. contributed equally to this work

E-mail: e.santos@qub.ac.uk 


\section{Table of Contents}

- Supplementary Section S1: Further analysis on the dielectric properties of 2D materials

- Supplementary Section S2: Hypothetical 2D "dielectric constant" rescaled from the 3D dielectric constant

- Supplementary Section S3: Polarizability-based theoretical model

- Supplementary Section S4: Dependence of $\alpha_{2 \mathrm{D}}$ on bandgap

- Supplementary Section S5: Validation of results using a larger 2D material database

- Supplementary Section S6: More discussion about the relation between 2D and 3D properties

- Supplementary Section S7: More discussions about the dielectric anisotropy

- Supplementary Section S8: Raw data from first principles calculations 


\section{S1 Further analysis on the dielectric properties of 2D materials}

In this section we provide more analysis on the dielectric properties of $2 \mathrm{D}$ materials calculated using many-body Green function method $\left(\mathrm{G}_{0} \mathrm{~W}_{0}\right)$, including electron-hole interactions at the level of the Bethe-Salpeter equation $\left(\mathrm{G}_{0} \mathrm{~W}_{0}-\mathrm{BSE}\right)$, and at the frequency-dependent regime.

\section{S1.1 Profile of induced dipoles of 2D material}

Here we show in detail the $\Delta \rho=\rho(\boldsymbol{E})-\rho(\boldsymbol{E}=0)$ profile of the $2 \mathrm{H}-\mathrm{MoS}_{2}$ slab in main text Figure 1. The density $\Delta \rho$ is calculated via $\Delta \rho(z)=\frac{1}{S} \int_{S} \Delta \rho(x, y ; z) d x d y$, where $S$ is the surface of the unit cell perpendicular to a given direction, in this case $z$. As can be seen in Figure 1 the induced charges on the $\mathrm{MoS}_{2}$ layer only extends to a width of $\sim 12 \AA$ centered at the middle of the layer. This corresponds to about 5-6 $\AA$ from each side. When the SL size $L \gg 12 \AA$ as in the first principle calculations shown in the main text, the induced dipoles from the periodic images do not interact thus giving the converged values of $\alpha_{2 \mathrm{D}}$.

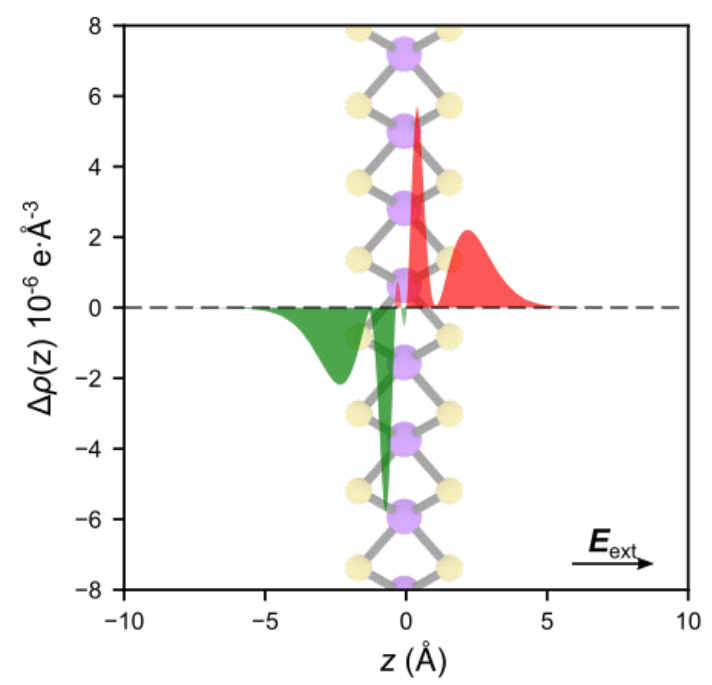

Figure 1: $\Delta \rho$ as a function of $z$ around the $\mathrm{MoS}_{2}$ layer, corresponding to main text Figure 1a. Green and red parts corresponding to negative and positive induced charges. The external electric field $E_{\text {ext }}$ is $0.01 \mathrm{eV} / \AA$. 


\section{S1.2 Dielectric properties calculated using many-body Green function method and frequency dependency}

Here we will show the results of dielectric properties calculated using many-body Green function method $\left(\mathrm{G}_{0} \mathrm{~W}_{0}\right)$ and with electron-hole interactions at the level of the Bethe-Salpeter equation $\left(\mathrm{G}_{0} \mathrm{~W}_{0}-\mathrm{BSE}\right)$. Frequency-dependent dielectric functions, $\varepsilon_{\mathrm{SL}}(\omega)$, were calculated at the level of $\mathrm{G}_{0} \mathrm{~W}_{0}$ and $\mathrm{G}_{0} \mathrm{~W}_{0}+\mathrm{BSE}$ levels using VASP. For the calculations on $\mathrm{G}_{0} \mathrm{~W}_{0}$, a $12 \times 12 \times 1 \Gamma$-centered k-grid was used along with a $800 \mathrm{eV}$ energy cutoff in the plane waves and in calculation of the response function. 120 bands ( 4 occupied and 116 unoccupied) were used in the calculation of $\varepsilon_{\mathrm{SL}}(\omega)$ of monolayer BN, with local-field effects being included. For the calculation of the BetheSalpeter equation, the Tamm-Dancoff approximation was used with two occupied and two unoccupied bands being included.

We first compare the case of a monolayer of $\mathrm{BN}$ within a varying superlattice $L$ calculated

using $\mathrm{PBE}$ and $\mathrm{G}_{0} \mathrm{~W}_{0}$ as shown in Figure 2. Both $\varepsilon_{\mathrm{SL}}^{\|}$and $\varepsilon_{\mathrm{SL}}^{\perp}$ do not converge as a function of $L$ despite of the separation utilized in the simulations (Figure 2a-c). However, corresponding 2D polarizabilities are almost $L$-independent (Figure $2 \mathbf{b}$-d). It is worth noting that since the $\mathrm{G}_{0} \mathrm{~W}_{0}$ method has better estimation of the electronic bandgap, $\varepsilon_{\mathrm{SL}}$ and $\alpha_{2 \mathrm{D}}$ are smaller using $\mathrm{G}_{0} \mathrm{~W}_{0}$ than in PBE functionals.

Next we will investigate the frequency-dependent dielectric properties of the 2D materials using various methods. The imaginary part of the frequency-dependent dielectric function of a periodic system is calculated using the following relation:

$$
\begin{array}{rl}
\varepsilon_{\alpha \beta}^{(2)}(\omega)=\frac{4 \pi e^{2}}{\Omega} \lim _{q \rightarrow 0} \frac{1}{q^{2}} \sum_{v, c, \mathbf{k}} & 2 \omega_{\mathbf{k}} \delta\left(\epsilon_{c \mathbf{k}}-\epsilon_{v \mathbf{k}}-\omega\right) \\
\times\left\langle u_{c \mathbf{k}+q \mathbf{e}_{\alpha}} \mid u_{v \mathbf{k}}\right\rangle\left\langle u_{v \mathbf{k}} \mid u_{c \mathbf{k}+q \mathbf{e}_{\beta}}\right\rangle
\end{array}
$$

Through the Kramers-Kronig transformation the real part of the dielectric function can be obtained 

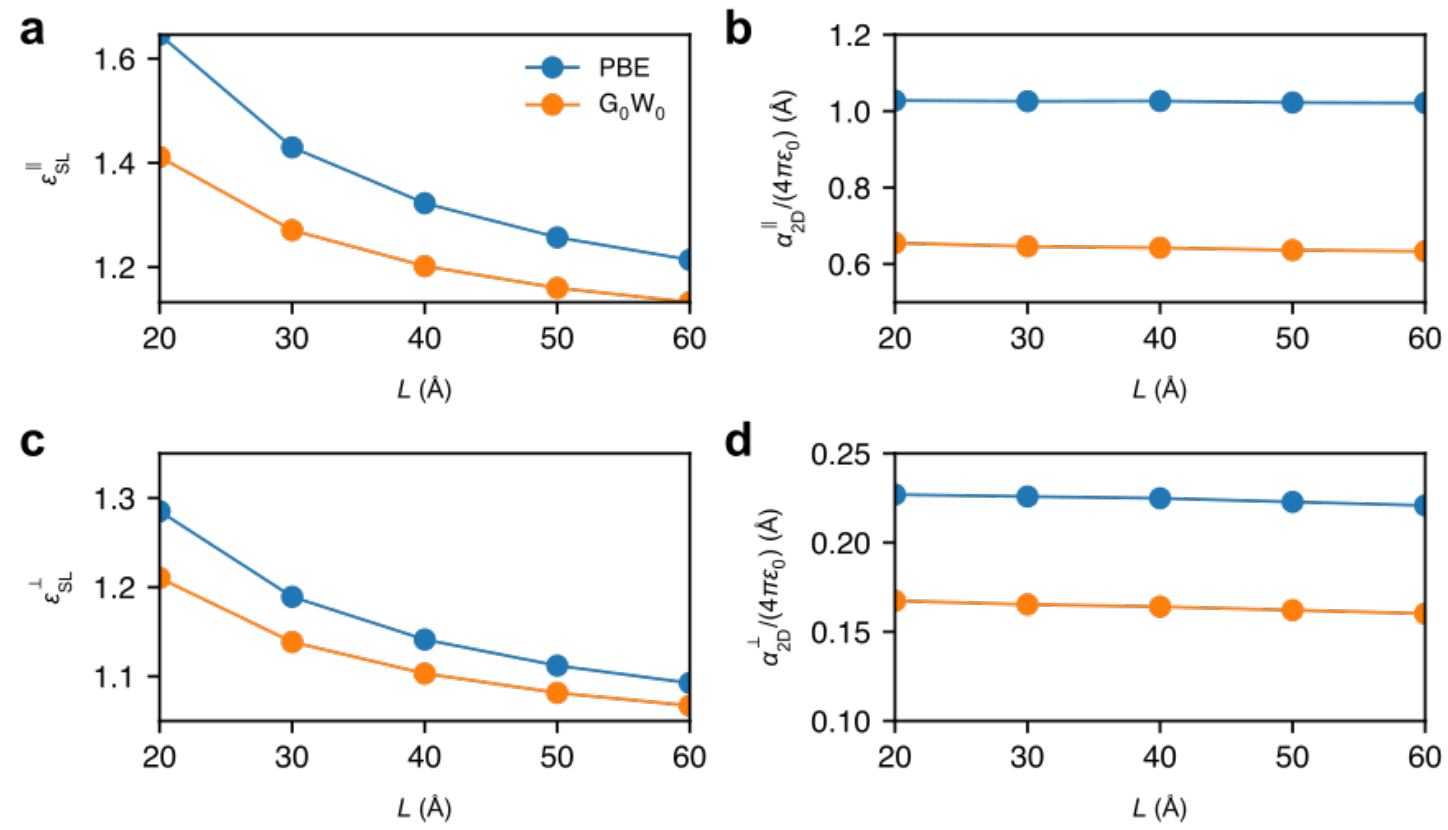

Figure 2: Variation of $\varepsilon_{\mathrm{SL}}^{\perp}$ as a function of $L$ for monolayer BN calculated at the level of PBE and $\mathbf{G}_{0} \mathbf{W}_{0}$. a-b. $\varepsilon_{\mathrm{SL}}^{\|}$and $\alpha_{2 \mathrm{D}}^{\|}$as function of $L$, respectively. c-d. $\varepsilon_{\mathrm{SL}}^{\perp}$ and $\alpha_{2 \mathrm{D}}^{\perp}$ as function of $L$. In all cases the values obtained using $\mathrm{G}_{0} \mathrm{~W}_{0}$ method is smaller than that using PBE method due to better estimation of the bandgap.

as:

$$
\varepsilon_{\alpha \beta}^{(1)}(\omega)=1+\frac{2}{\pi} \int_{0}^{\infty} \frac{\varepsilon_{\alpha \beta}^{(2)}\left(\omega^{\prime}\right) \omega^{\prime}}{\omega^{\prime 2}-\omega^{2}} d \omega^{\prime}
$$

We calculate the frequency-dependent dielectric properties of BN with varying superlattice $L$ at PBE, $\mathrm{G}_{0} \mathrm{~W}_{0}$, and $\mathrm{G}_{0} \mathrm{~W}_{0}-\mathrm{BSE}$. Figure 3 and 4 show that the magnitudes of $\varepsilon_{\mathrm{SL}}^{\|}(\omega)$ and $\varepsilon_{\mathrm{SL}}^{\frac{1}{}}(\omega)$ reduce with increasing $L$ throughout the whole frequency range at PBE level. The $L$-dependency can also be removed using main text Eqs. 2a and 2b, yielding lattice-independent polarizabilities throughout the frequency domain (Figure $3 \mathbf{b}-\mathbf{d}$ and $4 \mathbf{b}-\mathbf{d}$ ). Note that when extracting the frequency-dependent 2D polarizabilities from Eqs. $2 \mathrm{a}$ and $2 \mathrm{~b}$, the peak position in energy $\hbar \omega$ from $\varepsilon_{\mathrm{SL}}$ is preserved. This is explained by the fact that the local extrema from spectra of $\varepsilon_{\mathrm{SL}}$ is also the 
local extrema in corresponding $\alpha_{2 \mathrm{D}}$, since when $\partial \varepsilon_{\mathrm{SL}}(\omega) / \partial \omega=0$, we have

$$
\begin{aligned}
& \frac{\partial \alpha_{2 \mathrm{D}}^{\|}(\omega)}{\partial \omega}=\varepsilon_{0} L \frac{\partial \varepsilon_{\mathrm{SL}}^{\|}}{\partial \omega}=0 \\
& \frac{\partial \alpha_{2 \mathrm{D}}^{\perp}(\omega)}{\partial \omega}=\varepsilon_{0} L \frac{1}{\varepsilon_{\mathrm{SL}}^{2}} \frac{\partial \varepsilon_{\mathrm{SL}}^{\|}}{\partial \omega}=0
\end{aligned}
$$

which indicates that no corrections in energy are present when transforming $\varepsilon_{\mathrm{SL}}(\omega)$ to $\alpha_{2 \mathrm{D}}(\omega)$. Performing the simulations at the level of $\mathrm{G}_{0} \mathrm{~W}_{0}$ we observe blue shifts in energy in $\varepsilon_{\mathrm{SL}}(\omega)$ with increasing $L$. Figures 5 and 6 show that not only the magnitudes of the dielectric functions change with $L$ but also the peak positions. As a result the obtained polarizabilities also show $L$-dependent peak shift (Figures 5 b-d and 6b-d). This can be explained by the long-range nature of the Coulomb interactions into the self-energy $\Sigma=i G W$.

The non-interacting Green's function can be constructed as:

$$
G^{(0)}\left(\mathbf{r}, \mathbf{r}^{\prime}, \omega\right)=\sum_{n} \frac{\phi_{\mathrm{nk}}^{(0)}(\mathbf{r}) \phi_{\mathrm{nk}}^{*(0)}\left(\mathbf{r}^{\prime}\right)}{\omega-\epsilon_{\mathrm{n}}-+i \eta \operatorname{sgn}\left(\epsilon_{\mathrm{n}}-\epsilon_{\mathrm{F}}\right)}
$$

Here $\epsilon_{n}(\mathbf{k})$ are the DFT eigenenergies at $\mathbf{k}, \epsilon_{F}$ the Fermi energy, $\omega$ the frequency, $\left|u_{n \mathbf{k}}\right\rangle$ the cell periodic Bloch functions, $\phi_{n \mathbf{k}}$ are the one-electron orbitals and $\eta$ is an infintesimal complex shift. It can be seen from equation $\mathrm{S} 1$ there is a clear volume dependence on $\Omega$ of the dielectric function from the preceding DFT calculation. Thus, when carrying out a calculation on a slab, the dielectric function will vary with the vacuum spacing used.

Using the dielectric function we can calculate the screened Coulomb interaction:

$$
W=\epsilon^{-1} \nu
$$

where $\nu$ is the bare Coulomb interaction given by $e^{2} /\left|\mathbf{r}-\mathbf{r}^{\prime}\right|$. Due to the $1 /\left|\mathbf{r}-\mathbf{r}^{\prime}\right|$ term, images in the non-periodic direction have a long-range spurious interaction which varies with vacuum spacing. From the screened Coulomb interaction and non-interacting Green's function it is possible 
to calculate the self-energy of the system:

$$
\Sigma=i G W
$$

and the quasi-particle eigenenergies are found using:

$$
E_{\mathrm{nk}}^{\mathrm{QP}}=\Re\left[\left\langle\phi_{\mathrm{nk}}\left|-\frac{1}{2} \Delta+V_{\mathrm{ext}}+V_{\mathrm{H}}+\Sigma\left(\epsilon_{\mathrm{nk}}^{\mathrm{DFT}}\right)\right| \phi_{\mathrm{nk}}\right\rangle\right]
$$

Therefore a new set of eigenergies, $\mathrm{E}_{n \mathbf{k}}^{Q P}$ are found. Using $\mathrm{E}_{n \mathbf{k}}^{Q P}$ it is then possible to recalculate the dielectric function using the quasi-particle eigenergies as well as the DFT eigenfunctions.

In general terms excitonic effects are not taken into account at the level of PBE or $\mathrm{G}_{0} \mathrm{~W}_{0}$. For the former, this generally leads to an underestimation of the electronic bandgap and results in the first optical peak being lower in energy in comparison to more accurate methods. To go beyond PBE, the $\mathrm{G}_{0} \mathrm{~W}_{0}$ approximation replaces the exchange-correlation energy by the self-energy to include many-body effects through the interacting Green's function, $G$, and the screened Coulomb potential, $W$. This generally leads to an overestimation of the first optical peak as electron-hole coupling is not taken into account. This can be remedied by solving the Bethe-Salpeter equation using the eigenvalues obtained from $\mathrm{G}_{0} \mathrm{~W}_{0}\left(\mathrm{G}_{0} \mathrm{~W}_{0}\right.$ - BSE) which generally gives good agreement with experiment.

Due to the volume dependence of $\Sigma$ in equations S6 and S7, increasing the vacuum spacing leads to a change in the calculated quasi-particle eigenergies. This, along with the volume dependence in the calculation of the dielectric function, leads to an increase in energy of the peak position and a decrease in its magnitude with increasing vacuum spacing. Such effect is also discussed in several other studies, ${ }^{1,2}$ when full Coulomb interaction is used in a supercell. Including excitonic effects on top of a $\mathrm{G}_{0} \mathrm{~W}_{0}$ calculation through solving the Bethe-Salpeter equation correct this energy shift by localizing the exciton within the slab (Figures 7 and 8). However, the decrease in the magnitude of the dielectric function on both components of $\varepsilon_{\mathrm{SL}}(\omega)$ with increasing vacuum spacing is still observed. Using main text Eqs. 2a and $2 \mathrm{~b}$ we can remove the dependence on $L$ as 
plotted in Figures 7b-d and $8 \mathbf{b}-\mathbf{d}$ with fewer variations on $\varepsilon_{\mathrm{SL}}^{\perp}(\omega)$.

Combining the accuray of bandgap estimation, reproducible results of frequency-dependent dielectric properties and calculation efforts, the choice of HSE06 hybrid functional used in the main text provides best trade off between all aspects.
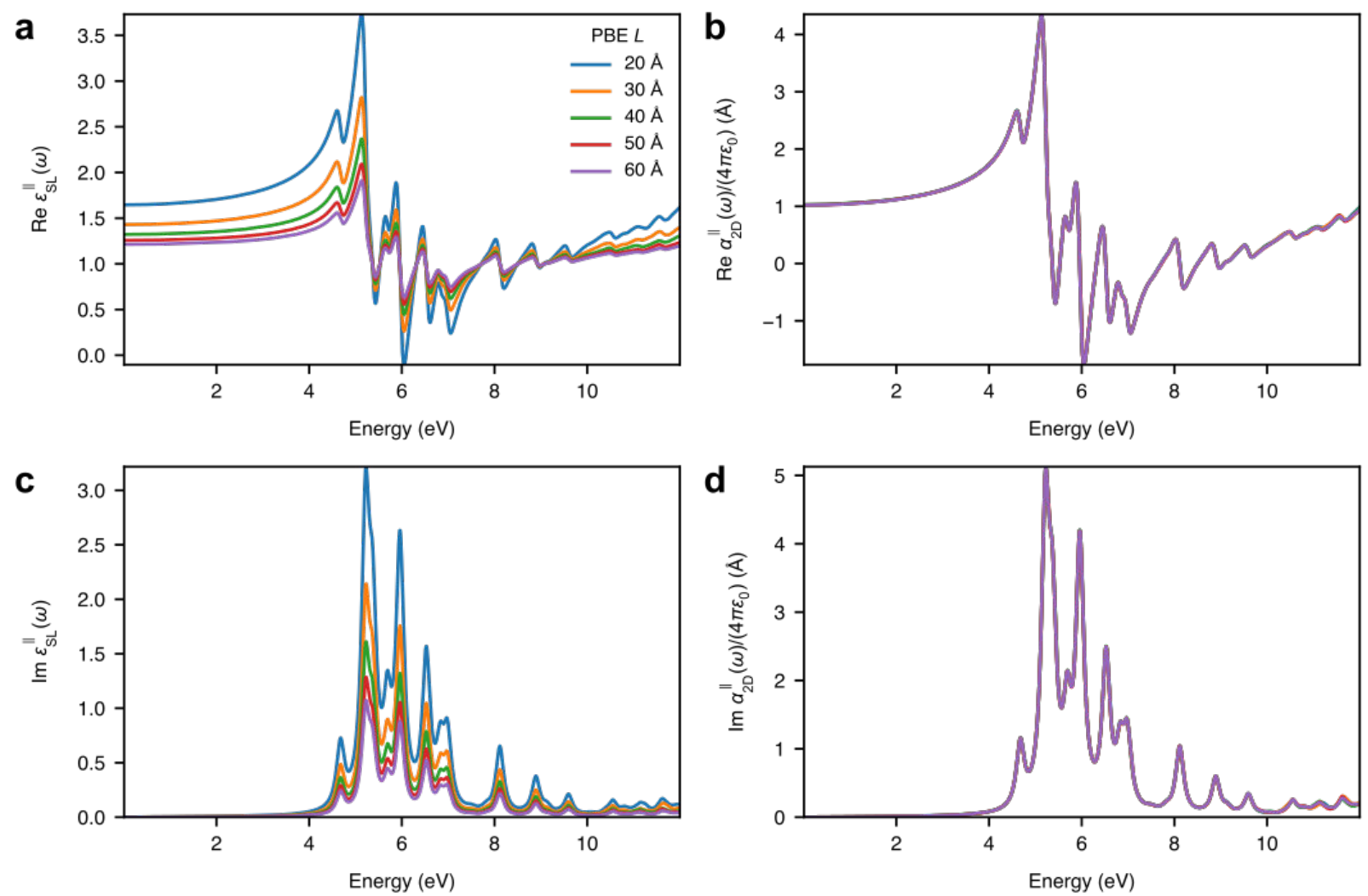

Figure 3: Dependence of in-plane dielectric properties on $L$ for monolayer BN calculated using PBE method: a Real part of $\varepsilon_{\mathrm{SL}}^{\|}$, b Real part of $\alpha_{2 \mathrm{D}}^{\|}$, $\mathbf{c}$ Imaginary part of $\varepsilon_{\mathrm{SL}}^{\|}$, $\mathbf{d}$ Imaginary part of $\alpha_{2 \mathrm{D}}^{\|}$. The same $\mathbf{k}$-sampling is used in all simulations with the only variable quantity being $L$. Clearly, the $L$-dependency of the superlattice dielectric function is removed using 2D polarizabilities. 

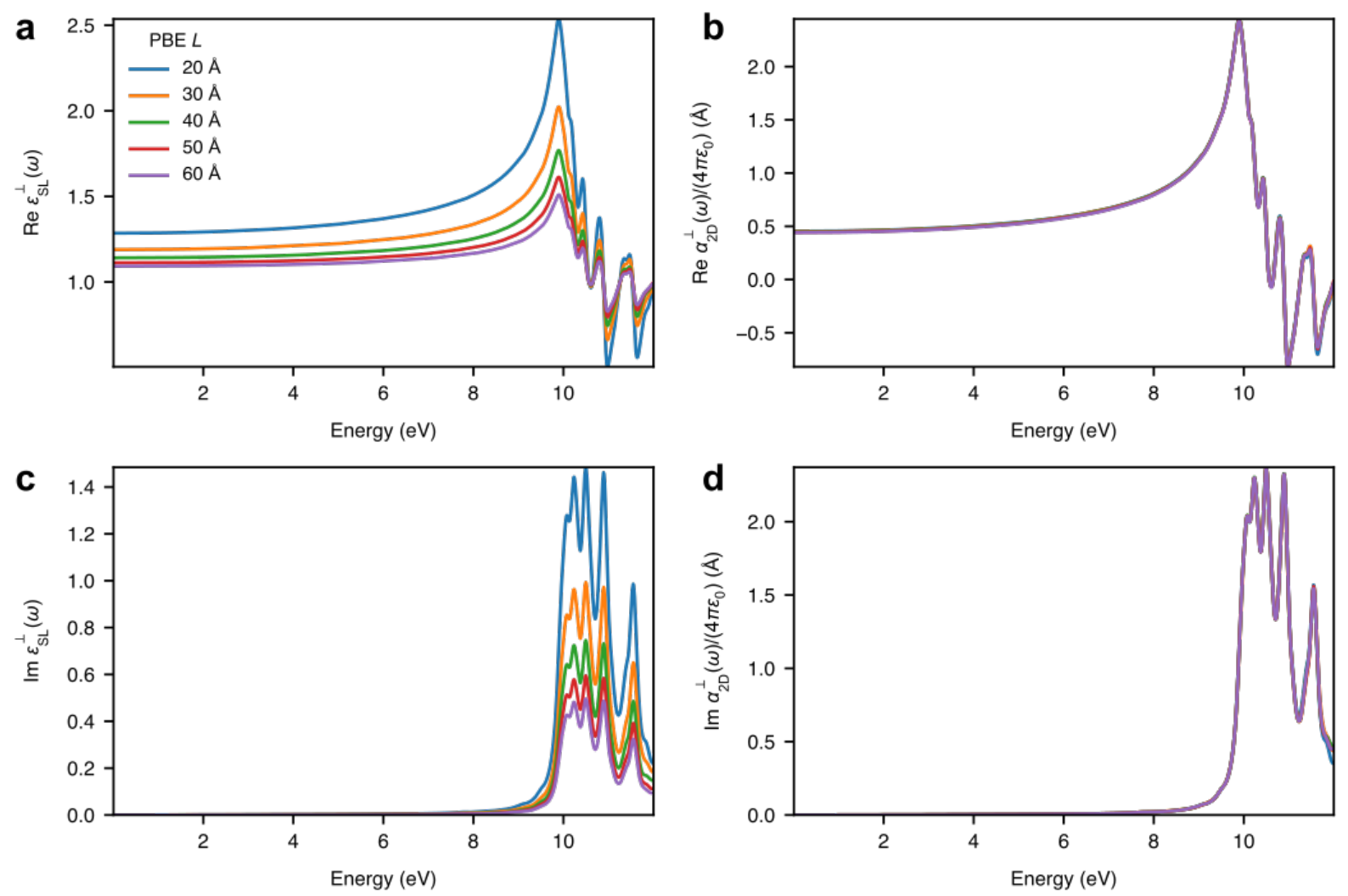

Figure 4: Dependence of out-of-plane dielectric properties on $L$ for monolayer BN calculated using PBE method: a Real part of $\varepsilon_{\mathrm{SL}}^{\perp}$, b Real part of $\alpha_{2 \mathrm{D}}^{\perp}$, $\mathbf{c}$ Imaginary part of $\varepsilon_{\mathrm{SL}}^{\perp}, \mathbf{d}$ Imaginary part of $\alpha_{2 \mathrm{D}}$. The same k-sampling is used in all simulations with the only variable quantity being $L$. Clearly, the $L$-dependency of the superlattice dielectric function is removed using $2 \mathrm{D}$ polarizabilities. 

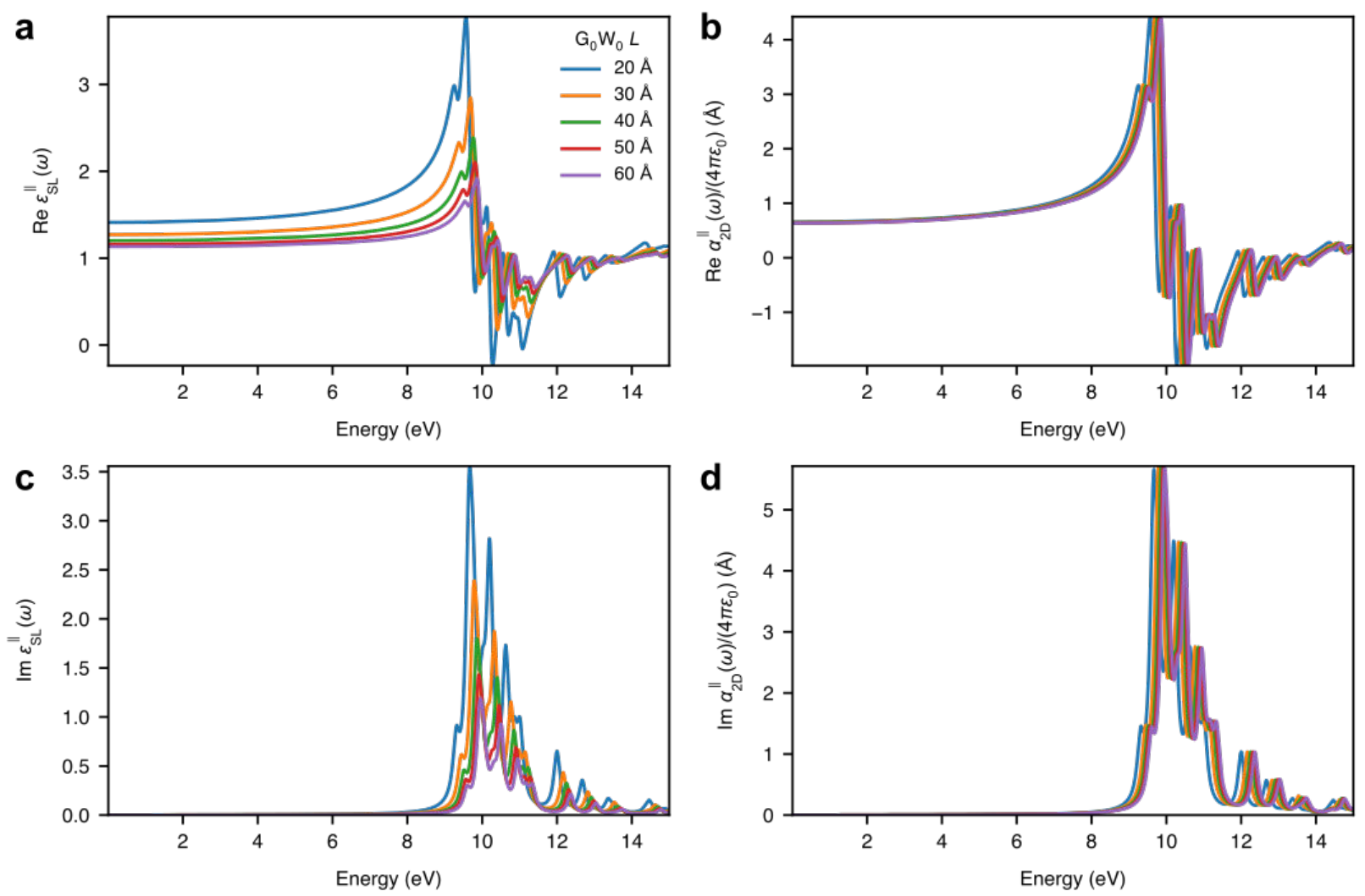

Figure 5: Similar to Figure 3 but calculated using $\mathrm{G}_{0} \mathrm{~W}_{0}$ method. A shift of peak position (frequency) in the dielectric function spectra is observed, resulted from the change of quasi-particle energy in $\mathrm{G}_{0} \mathrm{~W}_{0}$ calculations invloving the $2 \mathrm{D}$ slab. 

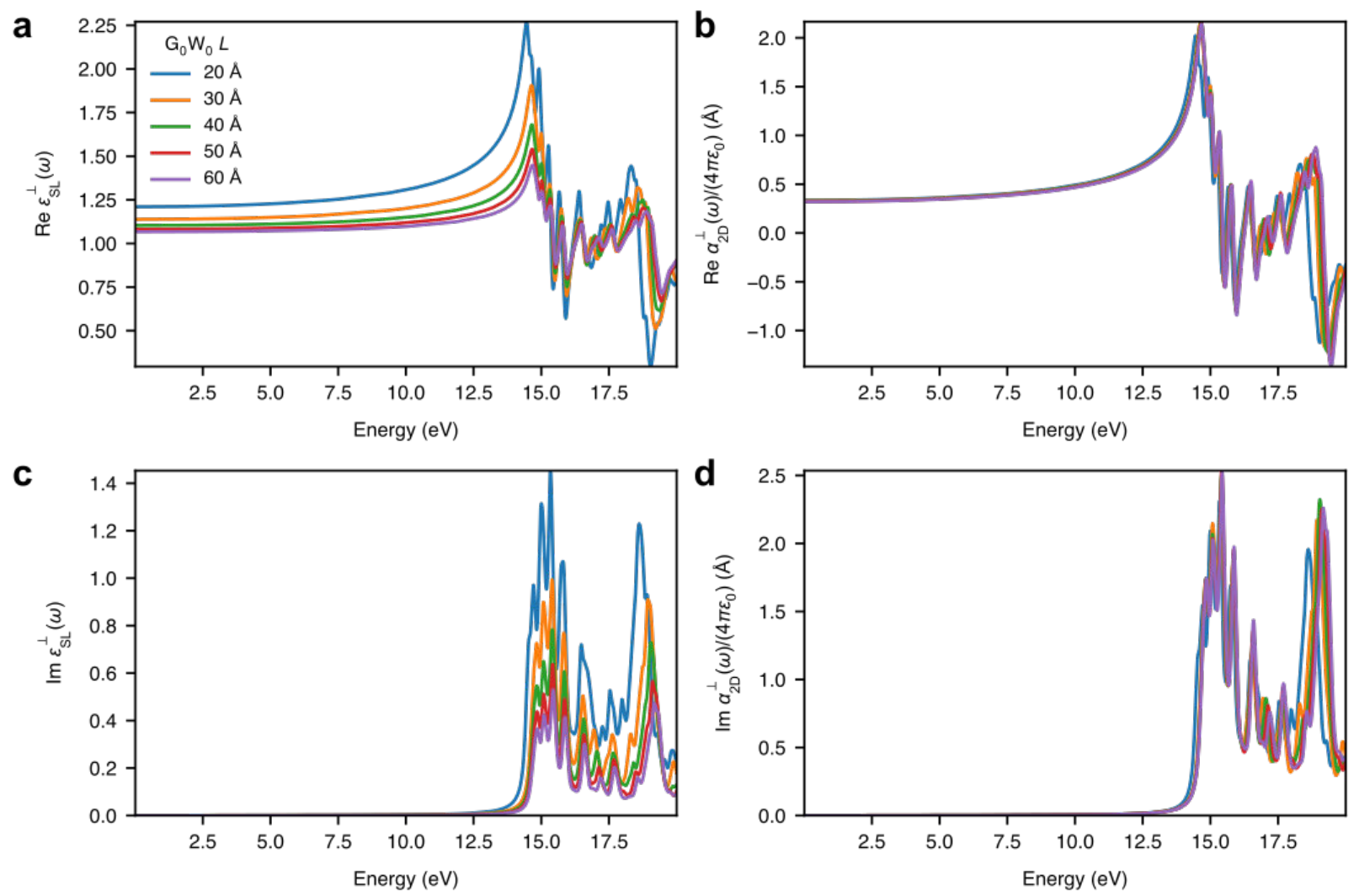

Figure 6: Similar to Figure 4 but calculated using $\mathrm{G}_{0} \mathrm{~W}_{0}$ method. A shift of peak position (frequency) in the dielectric function spectra is observed, resulted from the change of quasi-particle energy in $\mathrm{G}_{0} \mathrm{~W}_{0}$ calculations invloving the $2 \mathrm{D}$ slab. 

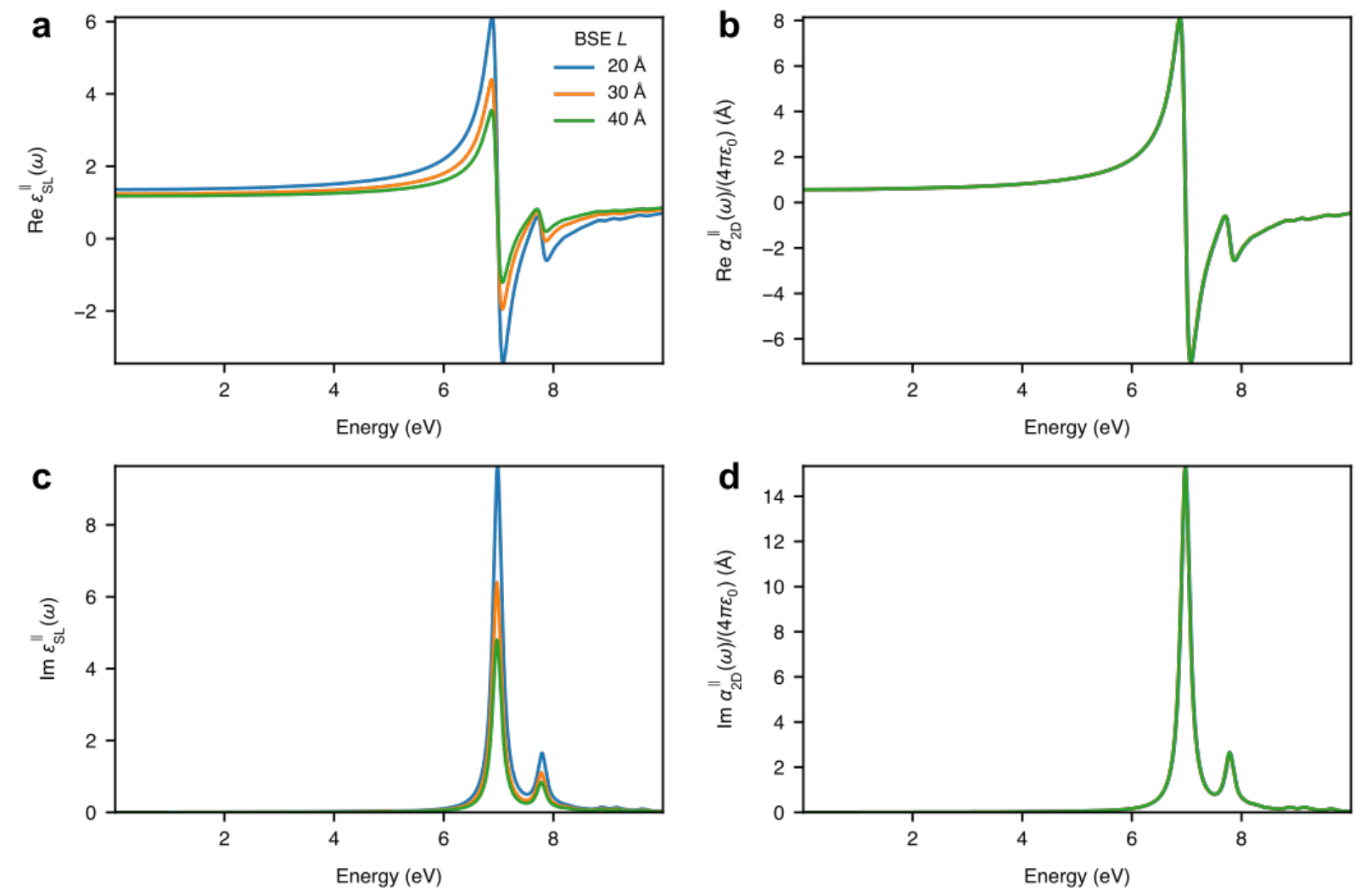

Figure 7: Similar as in Fig. 5 but taking into account electron-hole interactions at the level of the Bethe-Salpeter equation $\left(\mathrm{G}_{0} \mathrm{~W}_{0}+\mathrm{BSE}\right)$. The peak shift in energies are reduced in comparison with $\mathrm{G}_{0} \mathrm{~W}_{0}$. As a result the polarizabilities becomes almost independent of $L$, consistent to results obtained by the PBE method. 

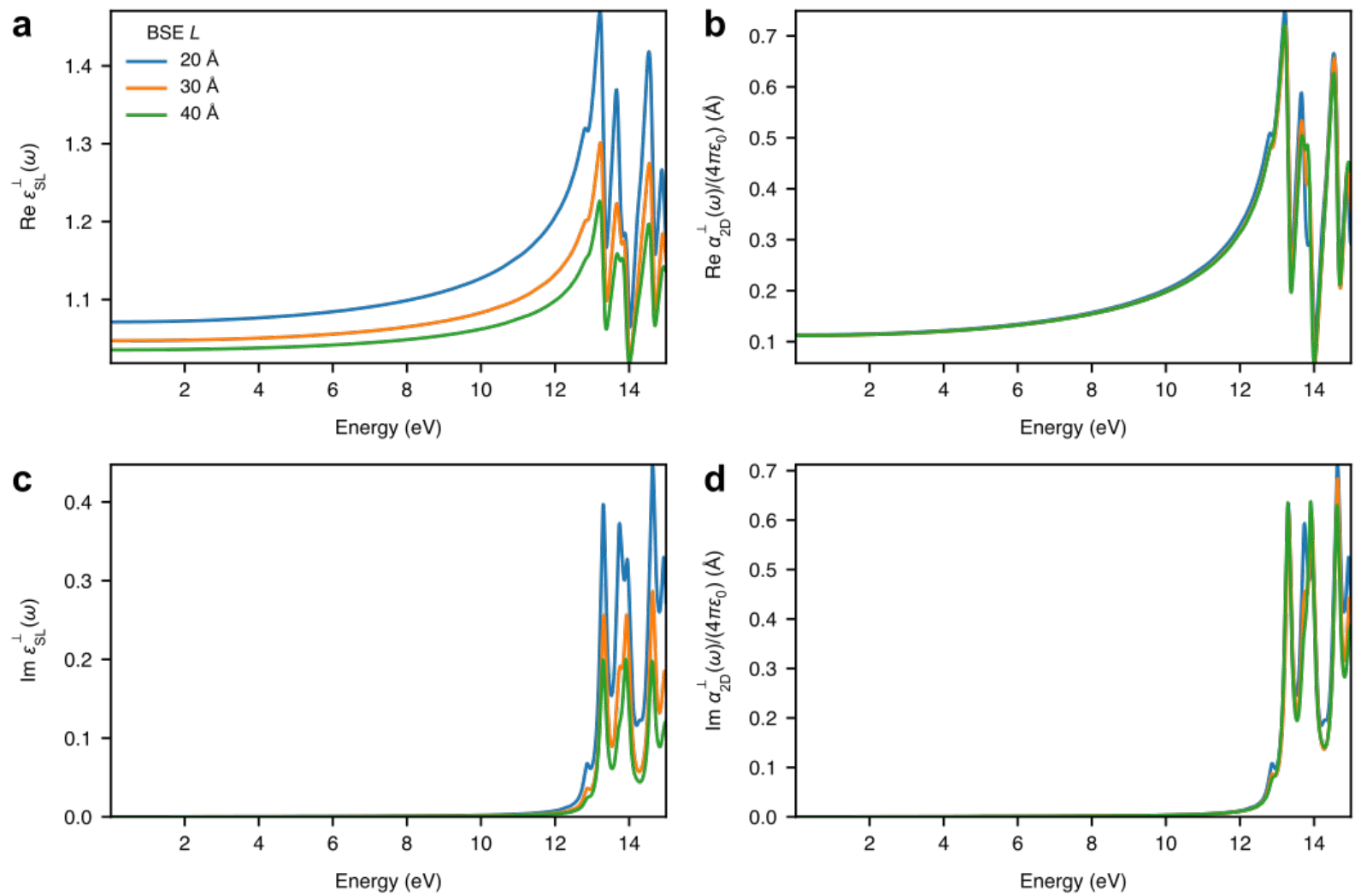

Figure 8: Similar as in Fig. 6 but taking into account electron-hole interactions at the level of the Bethe-Salpeter equation $\left(\mathrm{G}_{0} \mathrm{~W}_{0}+\mathrm{BSE}\right)$. As a result the polarizabilities becomes almost independent of $L$, consistent to results obtained by the PBE method.
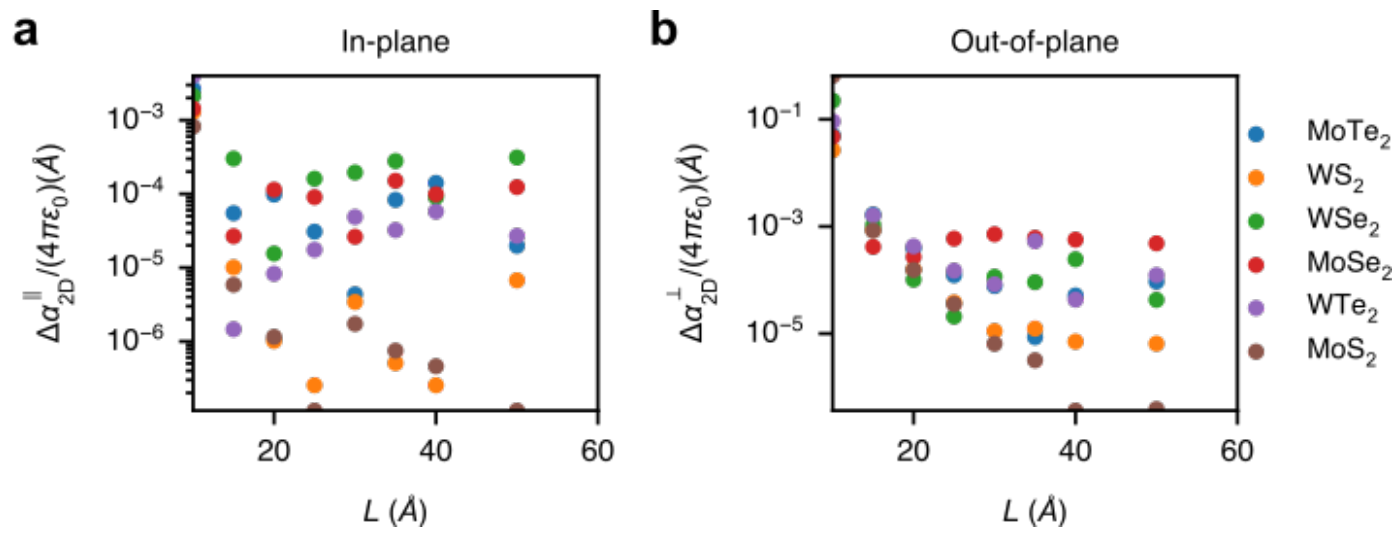

Figure 9: Convergence of electronic polarizabilities $\Delta \varepsilon_{2 \mathrm{D}}^{\|}(\mathbf{a})$ and $\Delta \varepsilon_{2 \mathrm{D}}^{\perp}(\mathbf{b})$ as functions of $L$ for the selected TMDCs, respectively. For both in- and out-of-plane polarizabilities, convergence is achieved when $L>15 \AA$ 


\section{S2 Further Data Concerning the Comparison with EDM}

The major issue when using rescale relations $3 \mathrm{a}-3 \mathrm{~b}$ comes from the determination of $\delta_{2 \mathrm{D}}^{*}$. To eliminate the modeling error caused by the a priori selection of this parameter, we perform the calculation of $\varepsilon_{\mathrm{SL}}$ of group 6 TMDCs against different $L$, and use least-square fitting to extract both $\varepsilon$ and $\delta$, as shown in Figure 10. The values of $\delta_{2 \mathrm{D}}^{*}$ extracted from both $\varepsilon_{\mathrm{SL}}^{\|}$and $\varepsilon_{\mathrm{SL}}^{\perp}$ are close when $L>15 \AA$. Notably, the $\delta_{2 \mathrm{D}}^{*}$ values are generally $10 \%$ smaller than the interlayer distance in corresponding bulk materials $L_{\mathrm{Bulk}}$, as shown in Table 1 . On the other hand, the extracted $\delta_{2 \mathrm{D}}^{*}$ values are closer to the covalent thickness $\delta_{\text {cov }}$ as described in the main text, with a difference generally smaller than $5 \%$. Our results indicate that the conventional estimation of the 2D layer thickness by its bulk interlayer distance, ${ }^{3,4}$ will always lead to overestimation. On the contrary, the out-of-plane polarizability $\alpha_{2 \mathrm{D}}^{\perp}$ correctly captures the thickness of $2 \mathrm{D}$ materials.

Table 1: Fitted effective thickness $\delta_{2 \mathrm{D}}^{\|, \text {fit }}$ and $\delta_{2 \mathrm{D}}^{\perp \text {, fit }}$ from in- and out-of-plane dielectric data, compared with the interlayer distance of corresponding bulk material $L_{\mathrm{Bulk}}$, the covalent thickness $\delta_{\text {cov }}$, and $\alpha_{2 \mathrm{D}}^{\perp} / \varepsilon_{0}$ for $2 \mathrm{H}$ TMDC materials.

\begin{tabular}{lccccc}
\hline Material & $\delta_{2 \mathrm{D}}^{\|, \text {fit }}(\AA)$ & $\delta_{2 \mathrm{D}}^{\perp, \text { fit }}(\AA)$ & $L_{\text {Bulk }}(\AA)$ & $\delta_{\text {cov }}(\AA)$ & $\alpha_{2 \mathrm{D}}^{\perp} / \varepsilon_{0}(\AA)$ \\
\hline $2 \mathrm{H}-\mathrm{MoS}_{2}$ & 5.76 & 5.49 & 6.15 & 5.22 & 4.98 \\
$2 \mathrm{H}-\mathrm{MoSe}_{2}$ & 5.98 & 5.92 & 6.46 & 5.73 & 5.60 \\
$2 \mathrm{H}-\mathrm{MoTe}_{2}$ & 6.43 & 6.85 & 6.98 & 6.37 & 6.12 \\
$2 \mathrm{H}-\mathrm{WS}_{2}$ & 5.63 & 5.49 & 6.15 & 5.20 & 5.00 \\
$2 \mathrm{H}-\mathrm{WSe}_{2}$ & 5.84 & 5.92 & 6.49 & 5.75 & 5.42 \\
$2 \mathrm{H}-\mathrm{WTe}_{2}$ & 6.32 & 6.58 & 7.06 & 6.38 & 6.33 \\
\hline
\end{tabular}

One main drawback of the EDM approach is the overestimation of the out-of-plane dielectric response. As can be seen in Figure 10, the extracted $\varepsilon_{2 \mathrm{D}}^{\perp}$ values for the TMDCs studied are comparable (within a range of $8-13 \%$ ) or even larger than $\varepsilon_{2 D}^{\|}$, which does not agree with the physical picture that electrostatic screening of 2D materials are much smaller perpendicular to the 2D plane. In fact, combining Eq. $3 b$ and the definition of $\alpha_{2 \mathrm{D}}^{\perp}$, we have:

$$
\frac{\alpha_{2 \mathrm{D}}^{\perp}}{\varepsilon_{0}}=\delta_{2 \mathrm{D}}^{*}\left(1-\left(\varepsilon_{2 \mathrm{D}}^{\perp}\right)^{-1}\right)
$$



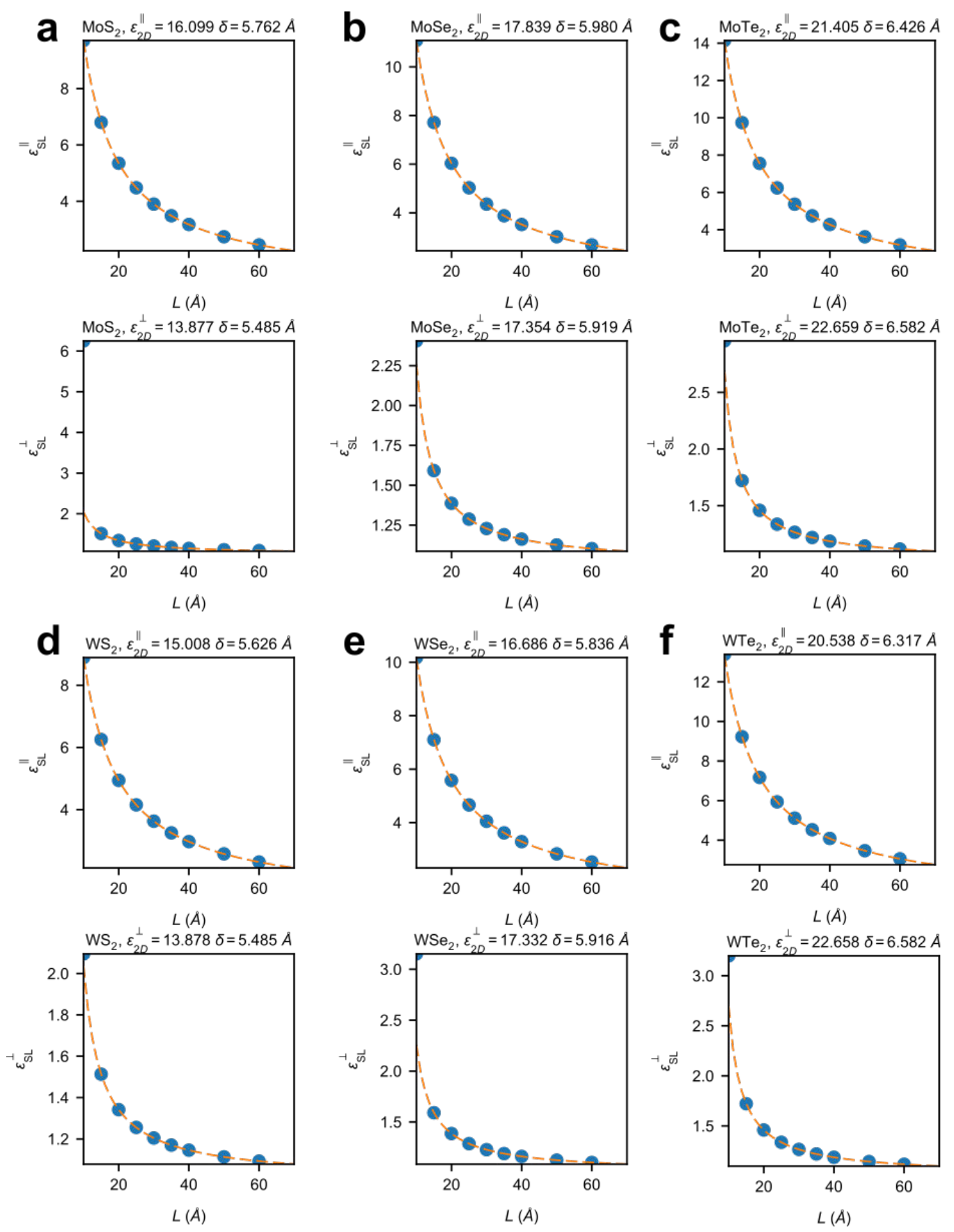

Figure 10: Calculated (blue dots) and fitted (orange broken lines) $\varepsilon_{\mathrm{SL}}$ as function of $L$ for the group 6 TMDCs: a. $\mathrm{MoS}_{2}$. b. $\mathrm{MoSe}_{2}$. c. $\mathrm{MoTe}_{2}$. d. $\mathrm{WS}_{2}$. e. $\mathrm{WSe}_{2}$. f. $\mathrm{WTe}_{2}$. The extracted values of $\varepsilon_{2 \mathrm{D}}$ and $\delta$ are shown in each subfigure. 
which indicates that the characteristic length $\alpha_{2 \mathrm{D}}^{\perp} / \varepsilon_{0}$, is very close but slightly smaller than $\delta_{2 \mathrm{D}}^{*}$ estimated by the effective medium theory, if $\varepsilon_{2 \mathrm{D}}^{\perp} \gg 1$. Moreover, from Eq. S8, when $\delta_{2 \mathrm{D}}^{*}$ and $\alpha_{2 \mathrm{D}}^{\perp} / \varepsilon_{0}$ are close, slight change of the $\delta_{2 \mathrm{D}}^{*}$ chosen may lead to divergence of $\varepsilon_{2 \mathrm{D}}^{\perp}$, as shown in Figure 11. Therefore cautions must be taken when treating the dielectric response of the $2 \mathrm{D}$ material using effective medium theory. In comparison, the 2D polarizability does not require the initial guess of the thickness.

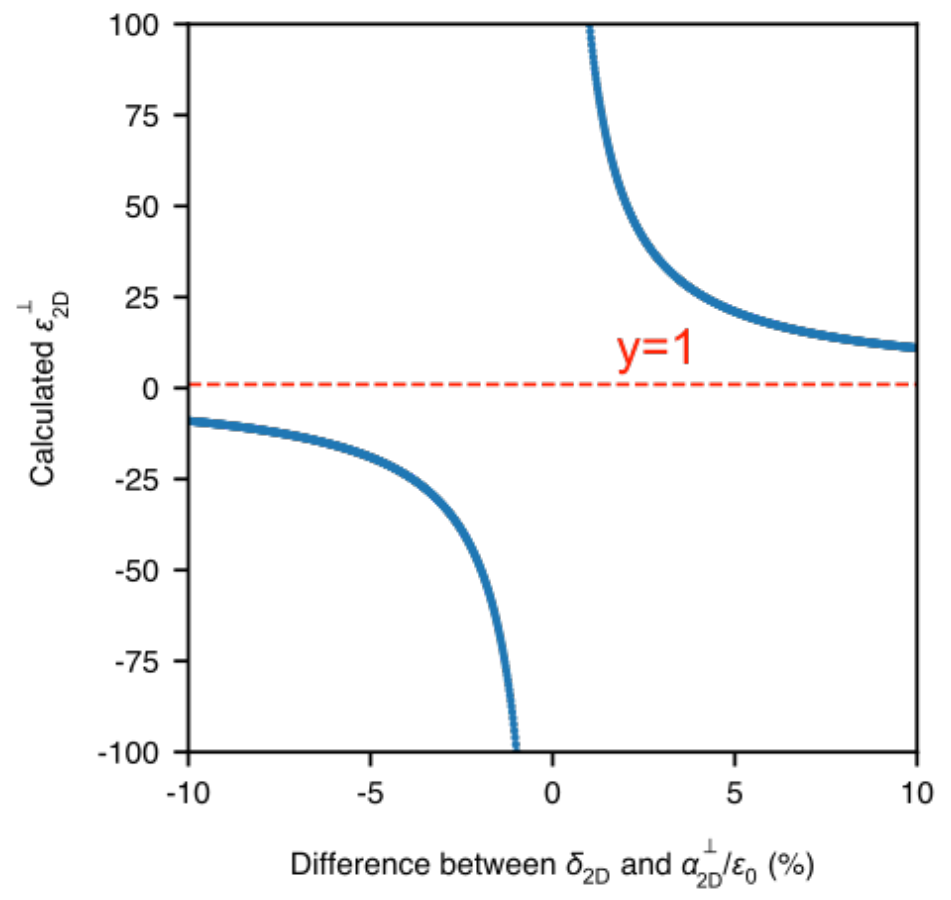

Figure 11: Calculated $\varepsilon_{2 \mathrm{D}}^{\perp}$ value as a function of the difference between $\delta_{2 \mathrm{D}}^{*}$ and $\alpha_{2 \mathrm{D}}^{\perp} / \varepsilon_{0}$. A small change of $\alpha_{2 \mathrm{D}}^{\perp} / \varepsilon_{0}$ chosen may lead to divergence of the $\varepsilon_{2 \mathrm{D}}^{\perp}$ or even negative values, which is apparently nonphysical.

To conclude, based on theoretical and technical considerations, there are several advantages of using the electronic polarizability for describing the dielectric nature of $2 \mathrm{D}$ materials, including:

1. $\alpha_{2 \mathrm{D}}$ can be used to describe both the local and macroscopic dielectric properties, while $\epsilon_{2 \mathrm{D}}$ is unable.

2. Calculating $\alpha_{2 \mathrm{D}}$ only requires to calculate the dielectric response at single superlattice length at relative small $L$, while $\epsilon_{2 \mathrm{D}}$ requires calculation with varied superlattice length. This is a 
big advantage on the computational side since the larger the supercell the larger the computational cost.

3. $\alpha_{2 \mathrm{D}}$ correctly represents the screening length of 2D material, while $\epsilon_{2 \mathrm{D}}$ calculated from EMT does not.

4. $\alpha_{2 \mathrm{D}}$ correctly represents the different degree of screening in/out-of-plane, while $\epsilon_{2 \mathrm{D}}$ does not.

5. The value of $\epsilon_{2 \mathrm{D}}$ hugely depends on the choice of the thickness of 2D material, while such information is intrinsically embedded in $\alpha_{2 \mathrm{D}}$.

\section{S2.1 Derivation of Eqs.2a and 2b}

To show that Eqs. $2 \mathrm{a}$ and $2 \mathrm{~b}$ do not use any arguments based on EMT but rather basic electrostatics, we show the derivation of both equations in the following.

\section{S2.1.1 Parallel to the surface}

For in-plane electric field, the electrostatic boundary conditions gives the continuity of the applied electric field as $E_{\mathrm{loc}}^{\|}=E^{\|},{ }^{5}$ which resulted in $P^{p}=\frac{1}{L} \mu_{2 \mathrm{D}}^{p}$. Indeed, $\mu_{2 \mathrm{D}}^{p}$ can be written in terms of the local electric field $E_{\mathrm{loc}}^{p}$ and the 2D polarizability $\alpha_{2 \mathrm{D}}$ as:

$$
\mu_{2 \mathrm{D}}^{p}=\alpha_{2 \mathrm{D}}^{\|} E_{\mathrm{loc}}^{\|}
$$

This gives for the in-plane polarization:

$$
P^{p}=\frac{1}{L} \alpha_{2 \mathrm{D}}^{\|} E_{\mathrm{loc}}^{\|}=\frac{1}{L} \alpha_{2 \mathrm{D}}^{\|} E^{\|}
$$

The derivative of this equation relative to the external field $E^{\|}$resulted in:

$$
\frac{\partial P^{p}}{\partial E^{\|}}=\frac{1}{L} \alpha_{2 \mathrm{D}}^{\|}
$$


Inserting this equation S11 into Eq.1a with $p=q=\|$ :

$$
\varepsilon_{\mathrm{SL}}^{\|}=1+\frac{\alpha_{2 \mathrm{D}}^{\|}}{\varepsilon_{0} L}
$$

\section{S2.1.2 Perpendicular to the surface}

The same procedure can be used along the out-of-plane component but using $E_{\mathrm{loc}}^{\perp}=E^{\perp}+\mu_{2 \mathrm{D}}^{\perp} / \varepsilon_{0} L$ for the local field. We can write this field as:

$$
E_{\mathrm{loc}}^{\perp}=E^{\perp}+\frac{\alpha_{2 \mathrm{D}}^{\perp} E_{\mathrm{loc}}^{\perp}}{\varepsilon_{0} L}
$$

If we re-arrange the terms for $E_{\text {loc }}^{\perp}$, we can ended up with:

$$
E_{\mathrm{loc}}^{\perp}=\frac{E^{\perp}}{1-\frac{\alpha_{2 \mathrm{D}}^{\perp}}{\varepsilon_{0} L}}
$$

We can write the polarization $P^{\perp}$ as:

$$
P^{\perp}=\frac{\alpha_{2 \mathrm{D}}^{\perp}}{L}\left(\frac{E^{\perp}}{1-\alpha_{2 \mathrm{D}}^{\perp} / \varepsilon_{0} L}\right)
$$

Taking the derivative of this equation relative to $E^{\perp}$ and inserting in Eq.1a with $p=q=\perp$ resulted in:

$$
\varepsilon_{\mathrm{SL}}^{\perp}=1+\frac{\alpha_{2 \mathrm{D}}^{\perp}}{\varepsilon_{0} L}\left(\frac{1}{1-\alpha_{2 \mathrm{D}}^{\perp} / \varepsilon_{0} L}\right)
$$

Re-arranging the terms, it ended up as:

$$
\varepsilon_{\mathrm{SL}}^{\perp}=\frac{\varepsilon_{0} L-\alpha_{2 \mathrm{D}}^{\perp}+\alpha_{2 \mathrm{D}}^{\perp}}{\varepsilon_{0} L-\alpha_{2 \mathrm{D}}^{\perp}}=\left(1-\frac{\alpha_{2 \mathrm{D}}^{\perp}}{\varepsilon_{0} L}\right)^{-1}
$$




\section{S3 Polarizability-Based Theoretical Model}

The universal relations for $\alpha_{2 \mathrm{D}}^{\|}$and $\alpha_{2 \mathrm{D}}^{\perp}$ revealed by Eqs. 4a and 4b are not coincidental. Combining recent theoretical findings of the linear relation between exciton binding energy $E_{\mathrm{b}}$ and $E_{\mathrm{g}}$ of $2 \mathrm{D}$ materials, ${ }^{6-8}$ and the fact that the $E_{\mathrm{b}}$ is roughly inversely proportional to $\alpha_{2 \mathrm{D}}^{\|},{ }^{9}$ it is reasonable to have a general relation between $\alpha_{2 \mathrm{D}}^{\|}$and $E_{\mathrm{g}}^{-1}$. Moreover, the bandgap-independent relation of $2 \mathrm{D} \alpha \frac{\perp}{2 \mathrm{D}}$ resembles molecular polarizabilities of conjugate molecules, ${ }^{10}$ fullerenes ${ }^{11}$ and carbon nanotubes, ${ }^{12}$ which are also shown to be geometry-dependent.

In this section we show in detail the polarizability-based theoretical framework that leads to the 2D Moss-like relations proposed in the main text. Due to its highly anisotropic nature, the wave function of an isolated 2D material $\psi(\mathbf{r})$ can be separated into the in- and out-of-plane components $\left(\psi^{\|}(\boldsymbol{\rho})\right.$ and $\left.\psi^{\perp}(z)\right)$ similar to the treatment of quantum wells (QW), ${ }^{13}$ such that $\psi=\psi^{\|} \psi^{\perp}$, where $\boldsymbol{\rho}=(x, y)$ is the in-plane coordinate. Using the Bloch theorem, the periodic $\psi^{\|}(\boldsymbol{\rho})$ can be further expressed as $\psi^{\|}(\boldsymbol{\rho})=e^{i \mathbf{k} \cdot \boldsymbol{\rho}} u(\boldsymbol{\rho})$, where $\mathbf{k}$ is the in-plane wave vector and $u(\boldsymbol{\rho})$ is periodic function in the xy-plane. According to the random phase approximation (RPA) theory, ${ }^{14} \varepsilon_{\mathrm{SL}}$ is the $\mathbf{q} \rightarrow 0$ and $\omega \rightarrow 0$ limits of the non-interacting dielectric function $\varepsilon(\mathbf{q}, \omega)$, where $\mathbf{q}$ is the momentum transfer and $\omega$ is the frequency:

$$
\varepsilon_{\mathrm{SL}}=\lim _{\mathbf{q} \rightarrow 0} 1+\frac{2 e^{2}}{\varepsilon_{0}|\mathbf{q}|^{2} \Omega} \sum_{\mathbf{k}, \mathrm{c}, \mathrm{v}} \frac{\left|<\psi_{\mathrm{v}}(\mathbf{k})\right| e^{-i \mathbf{q r}}\left|\psi_{\mathrm{c}}(\mathbf{k}+\mathbf{q})>\right|^{2}}{E_{\mathrm{c}}(\mathbf{k}+\mathbf{q})-E_{\mathrm{v}}(\mathbf{k})}\left[f\left(\psi_{\mathrm{c}}\right)-f\left(\psi_{\mathrm{v}}\right)\right]
$$

where $e$ is the unit charge, $c, v$ are the conduction and valence bands, $E$ is the eigenenergy of individual bands, and $f$ is the Fermi-Dirac distribution function. Taking the limit that $L \rightarrow \infty$, when $\varepsilon_{\mathrm{SL}}^{\perp} \approx 1$, we have $1-1 / \varepsilon_{\mathrm{SL}}^{\perp} \approx\left(\varepsilon_{\mathrm{SL}}^{\perp}-1\right)$. Therefore $\alpha_{2 \mathrm{D}}^{\|}$and $\alpha_{2 \mathrm{D}}^{\perp}$ at $0 \mathrm{~K}$ can be unified by the same equation:

$$
\alpha_{2 \mathrm{D}}=\frac{2 e^{2}}{|q|^{2} A} \sum_{\mathrm{k}, \mathrm{c}, \mathrm{v}} \frac{\left|<\psi_{\mathrm{v}}(\mathbf{k})\right| e^{-i \mathbf{q r}}\left|\psi_{\mathrm{c}}(\mathbf{k}+\mathbf{q})>\right|^{2}}{E_{\mathrm{c}}(\mathbf{k}+\mathbf{q})-E_{\mathrm{v}}(\mathbf{k})}
$$

where the direction is determined by q. Next we will show that the different behavior of $\psi^{\|}$and 
$\psi^{\perp}$ give rise to the main text Eqs. $4 \mathrm{a}$ and $4 \mathrm{~b}$.

\section{S3.1 Detailed derivations of main text Eq. 4a}

In this section we show how Eq. 2a is derived from Eq. S19. For the in-plane component $\alpha_{2 \mathrm{D}}^{\|}, e^{-i \mathbf{q r}}$ is independent of $z$, therefore the integral in $\left|<\psi_{\mathbf{v}}(\mathbf{k})\right| e^{-i \mathbf{q r}}\left|\psi_{\mathrm{c}}(\mathbf{k}+\mathbf{q})>\right|^{2}$ becomes independent of $\psi^{\perp}$, due to the orthogonality and normalization. The Bloch-wave form of $\psi^{\|}$ensures that only the cell-function $u(\mathbf{k})$ contributes to the final result of $\alpha_{2 \mathrm{D}}^{\|},{ }^{13}$ such that:

$$
\alpha_{2 \mathrm{D}}^{\|}=\frac{2 e^{2}}{(2 \pi)^{2}} \int d^{2} \mathbf{k} \sum_{\mathrm{c}, \mathrm{v}} \frac{\left|<u_{\mathrm{c}}(\mathbf{k})\right| \nabla\left|u_{\mathrm{v}}(\mathbf{k})>\right|^{2}}{E_{\mathrm{c}}(\mathbf{k})-E_{\mathrm{v}}(\mathbf{k})}
$$

Following the method of $\mathbf{k} \cdot \mathbf{p}$ theory from Ref. 8, the matrix element in the numerator of Eq. S20 is approximated by:

$$
\left|<u_{\mathrm{c}}(\mathbf{k})\right| \nabla\left|u_{\mathrm{v}}(\mathbf{k})>\right|^{2} \approx \frac{\hbar^{2}}{2 m^{*}} \frac{1}{E_{\mathrm{g}}+\frac{\hbar^{2} k^{2}}{2 m^{*}}}
$$

plug it into Eq. S20 and integrate within the 2D Brillouin zone from $|k|=0$ to $|k|=k_{\mathrm{BZ}}$, where $k_{\mathrm{BZ}}$ is the wavevector at the boundary of the 2D Brillouin Zone, we get:

$$
\begin{aligned}
\alpha_{2 \mathrm{D}}^{\|} & =N \cdot-\left.\frac{e^{2}}{2 \pi} \frac{1}{E_{g}+\beta}\right|_{\beta=0} ^{\beta=\frac{\hbar^{2} k_{\mathrm{BZ}}^{2}}{m^{*}}} \\
& \approx N e^{2} /\left(2 \pi E_{\mathrm{g}}\right)=C^{\|} E_{g}^{-1}
\end{aligned}
$$

where $N$ is degeneracy of bands associated with $E_{g}$. The approximation in Eq. S22 is due to the fact that $\frac{\hbar^{2} k_{\mathrm{BZ}}^{2}}{m^{*}} \gg E_{g}$, and we arrive at Eq. $4 \mathrm{a}$.

The coefficient of $C^{\|}$adapted from Ref. $8 C^{\|}=N e^{2} /(2 \pi)$ predicts linear correlation between

$\alpha_{2 \mathrm{D}}^{\|}$and $E_{\mathrm{g}}^{-1}$. We validate this by examining the DFT-calculated $\left(4 \pi \varepsilon_{0}\right) / \alpha_{2 \mathrm{D}}^{\|}$(measured in $\AA^{-1}$ ) and $E_{\mathrm{g}}$ (measured in eV) in Figure 2c. The coefficient $C^{\|}$becomes $8 \pi^{2} \varepsilon_{0} /(e N) \approx 0.436 / N=$ 0.183 , corresponding to $N$ between 2 and 3, which is a reasonable result for the $2 \mathrm{D}$ materials 
studied.

\section{S3.2 Detailed derivation of main text Eq. 4b}

For main text Eq. 4b, treating the in-plane wave functions as plane wave with form $\psi^{\|}(\rho) \propto e^{i \mathbf{k} \rho}$, the matrix element of $\left\langle\psi_{\mathrm{v}}(\mathbf{k})\left|e^{-i \mathbf{q r}}\right| \psi_{\mathbf{c}}(\mathbf{k}+\mathbf{q})>\right.$, when $\mathbf{q}=\left(0,0, q_{z}\right)$, becomes: ${ }^{15}$

$$
\begin{aligned}
<\psi_{\mathrm{v}}(\mathbf{k})\left|e^{-i \mathbf{q r}}\right| \psi_{\mathrm{c}}(\mathbf{k}+\mathbf{q})> & =\frac{1}{A} \int d x \int d y e^{i(-\mathbf{k} \rho-\mathbf{q} \rho+(\mathbf{k}+\mathbf{q}) \rho)} \int\left(\psi^{\perp}\right)_{\mathrm{v}}^{*}(\mathbf{k}) e^{-i q_{z} z} \psi_{\mathrm{c}}^{\perp}(\mathbf{k}+\mathbf{q}) \\
& =<\psi_{\mathrm{v}}^{\perp}(\mathbf{k})\left|e^{-i q_{z} z}\right| \psi_{\mathrm{c}}^{\perp}(\mathbf{k}+\mathbf{q})>
\end{aligned}
$$

Note that the states perpendicular are bound, the integral is meaningful only when $\mathbf{k}=\mathbf{k}+\mathbf{q}^{13}$ By performing the Taylor expansion of $e^{-i \mathbf{q r}} \approx 1-i \mathbf{q r}$, we get:

$$
\begin{aligned}
<\psi_{\mathrm{v}}^{\perp}(\mathbf{k})\left|e^{-i q_{z} z}\right| \psi_{\mathrm{c}}^{\perp}(\mathbf{k})> & \approx<\psi_{\mathrm{v}}^{\perp}(\mathbf{k})\left|\psi_{\mathrm{c}}^{\perp}(\mathbf{k})>-i q_{z}<\psi_{\mathrm{v}}^{\perp}(\mathbf{k})\right| z \mid \psi_{\mathrm{c}}^{\perp}(\mathbf{k})> \\
& =-i q_{z}<\psi_{\mathrm{v}}^{\perp}(\mathbf{k})|z| \psi_{\mathrm{c}}^{\perp}(\mathbf{k})>
\end{aligned}
$$

plug this into Eq. S19 and express the summation over $k_{x}$ and $k_{y}$ in a continuous form within the Brillouin Zone, we arrive at:

$$
\alpha_{2 \mathrm{D}}^{\perp}=\frac{2 e^{2}}{(2 \pi)^{2}} \int d^{2} \mathbf{k} \sum_{\mathrm{c}, \mathrm{v}} \frac{\left|<\psi_{\mathrm{v}}(\mathbf{k})\right| z\left|\psi_{\mathrm{c}}(\mathbf{k})>\right|^{2}}{E_{\mathrm{c}}(\mathbf{k})-E_{\mathrm{v}}(\mathbf{k})}
$$

The formalism is slightly different from Eq.S20.

The out-of-plane wave function $\psi^{\perp}(z)$ is the solution to the Schrödinger equation with Hamiltonian $\mathcal{H}=-\hbar^{2} \nabla^{2} / 2 m_{e}+V(z)$, where $\hbar$ is the reduced Planck constant, $m_{e}$ is electron mass and $V(z)$ is the confined Coulomb potential along the z-direction created by the nuclei. ${ }^{13,16}$ Although the exact form for $\psi^{\perp}$ depends on the exact distribution of $V(z)$, without loss of generality we can assume the electrons are confined in a potential well of width $\delta$, which is the typical treatment for semiconductor QWs. ${ }^{16-18}$ The allowed bound states inside the confined region generally have wave vector $k_{z} \propto n \pi / \delta$. With the total energy $E_{n}(\mathbf{k})=\frac{\hbar^{2}\left(k_{x}^{2}+k_{y}^{2}\right)}{2 m^{\|}}+\frac{\hbar^{2} n^{2} \pi^{2}}{2 m^{\perp} \delta^{2}}$, where $m^{\|}$and $m^{\perp}$ are 
the effective masses parallel and perpendicular to the 2D plane. Therefore, the denominator of Eq. $\mathbf{S} 25$ becomes independent of $\mathbf{k}$, that $E_{\mathrm{c}}(\mathbf{k})-E_{\mathrm{v}}(\mathbf{k})=\left(n_{\mathrm{c}}^{2}-n_{\mathrm{v}}^{2}\right) \frac{\hbar^{2} \pi^{2}}{2 m^{\perp} \delta^{2}}$. On the other hand, the numerator $<\psi_{\mathrm{v}}^{\perp}(\mathbf{k})|z| \psi_{\mathrm{c}}^{\perp}(\mathbf{k})>$ is proportional to the confinement length $\delta$ which can be seen using the particle-in-box solution. ${ }^{13}$ In combination, the individual terms of the summation in the right hand of Eq. S25 is independent of neither $E_{\mathrm{g}}$ nor $\mathbf{k}$, proving that $\alpha_{2 \mathrm{D}}^{\perp}$ is independent of the band gap. In the next section we will provide a simple explanation for the $\alpha \propto \delta$ relation from fundamental electrostatics theory.

\section{S3.3 Explanation of main text Eq. 4b from fundamental electrostatics}

The dependency of $\alpha_{2 \mathrm{D}}^{\perp}$ on the thickness $\delta$ of a 2D material, can also be regarded using fundamental electrostatic model. Consider the smallest repeating unit of the 2D material with xy-plane area $A$, under small perturbation field $E$ along the z-direction. Note that the surface bound charge $\sigma_{\mathrm{b}}=n e / A$, where $n$ is the number of unit charges contributes to the bound charges, comes only from the dipoles of the outer-most atoms, since the induced charges from inner atoms are cancel out (see Figure 12). From the definition of $\alpha_{2 \mathrm{D}}^{\perp}$, we have:

Dipole from individual atoms

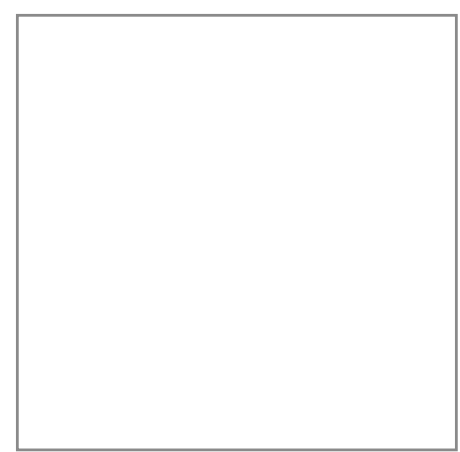

Dipole from outer-most atoms

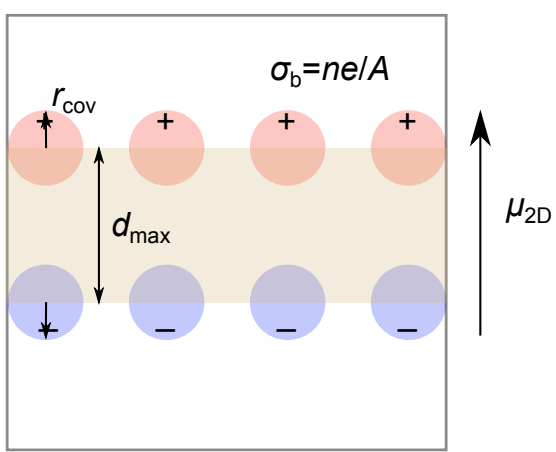

Figure 12: Fundamental electrostatic model for the thickness-dependency of $\alpha_{2 \mathrm{D}}^{\perp}$, using $2 \mathrm{H}-\mathrm{MoS} 2$ as an example. Left: induced dipoles from individual atoms along the $\mathrm{z}$-direction. The positive and negative induced charges from inner atoms cancel out. Right: simplified model for the thickness dependency of $\alpha_{2 \mathrm{D}}^{\perp}$, where the surface dipole density $\boldsymbol{\mu}$ comes only from the outer-most atoms.

$$
\alpha_{2 \mathrm{D}}^{\perp}=\frac{\boldsymbol{u}_{z}}{E_{\mathrm{loc}} A}=\frac{\left(d_{\mathrm{max}}+r_{\mathrm{cov}}^{i}+r_{\mathrm{cov}}^{j}\right) \sigma_{\mathrm{b}}}{E}
$$


where $r_{\mathrm{cov}}^{i}$ and $r_{\mathrm{cov}}^{j}$ are the covalent radii of the outer-most atoms, the characteristic length of the dipole extension in z-direction, respectively, and $d_{\max }$ is the z-distance between the nuclei of such atoms. The field $E$ counterbalances the field from the surface bound charges and equals $E=\sigma_{\mathrm{b}} / \varepsilon_{0}$. Therefore we have:

$$
\alpha_{2 \mathrm{D}}^{\perp}=\left(d_{\max }+r_{\mathrm{cov}}^{i}+r_{\mathrm{cov}}^{j}\right) \varepsilon_{0}=\delta_{\mathrm{cov}} \varepsilon_{0}
$$

which explains the linear relation seen in main text Figure 2d. We can see that such simple model nicely captures the thickness feature of $\alpha_{2 \mathrm{D}}^{\perp}$, and reproduces the right coefficient between $\delta_{\text {cov }}$ and $\alpha_{2 \mathrm{D}}^{\perp}$.

\section{S4 Dependence of $\alpha_{2 \mathrm{D}}$ on bandgap}

In this section we further look into the bandgap dependency of the 2D polarizability. Figure 13 shows the raw data of $\alpha_{2 \mathrm{D}}^{\|}$and $\alpha_{2 \mathrm{D}}^{\perp}$ as functions of $E_{\mathrm{g}}$ of the 2D materials investigated. We observe that $\alpha_{2 \mathrm{D}}^{\|}$can be approximated by a reciprocal function of $E_{\mathrm{g}}$, that $\alpha_{2 \mathrm{D}}^{\|} \sim 7.295\left(E_{\mathrm{g}}\right)^{-1}$. On the other hand, the plot of $\alpha_{2 \mathrm{D}}^{\perp}$ against $E_{\mathrm{g}}$ shows no apparent correlation.

We also investigate the relation of $2 \mathrm{D}$ polarizabilities with difference choices of ab initio bandgaps. It is widely accepted that the PBE exchange correlation, tends to underestimate the bangap. ${ }^{19-21}$ Indeed, changing the choice of $E_{\mathrm{g}}$ yields different regression relation with $1 / \alpha_{2 \mathrm{D}}^{\|}$, as shown in Figure 14. We see that due to the underestimation of PBE bandgap, the slope of linear regression is larger than that from HSE-bandgap. We also observe that the $1 / \alpha-E_{\mathrm{g}}$ relation is better presented by using the minimal HSE bandgap than the minimal PBE bandgap, due to higher regression $R^{2}$ coefficient of the former. We note that the higher $R^{2}$ coefficient observed using the direct PBE bandgap than the minimal PBE bandgap may be solely caused by the fact that the direct bandgap of 2D materials on the PBE level is closer to the HSE bandgap. From the random phase approximation theory of dielectric response, the polarizability is contributed by all possible transition between valence and conduction bands, with the minimal bandgap as the least possible 
a

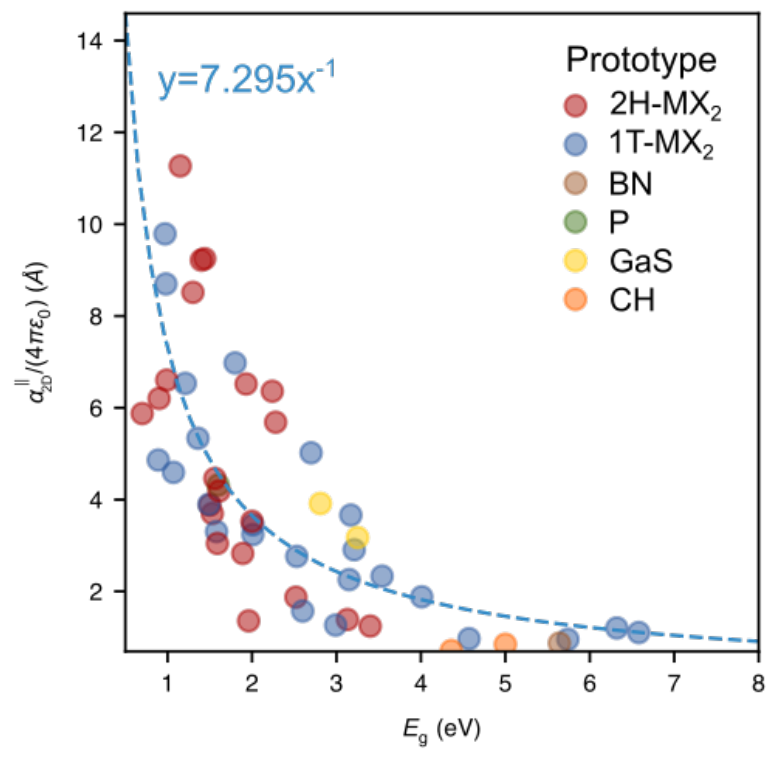

b

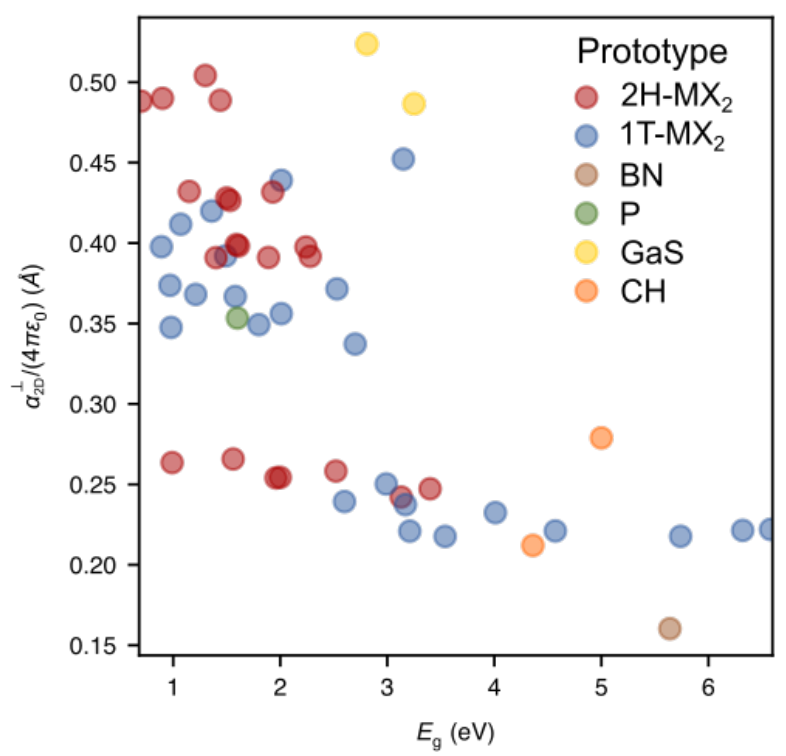

Figure 13: $E_{\mathrm{g}}$-dependence of $\mathbf{a}, \alpha_{2 \mathrm{D}}^{\|}$and $\mathbf{b}, \alpha_{2 \mathrm{D}}^{\perp}$ for the $2 \mathrm{D}$ materials investigated here using HSE06.

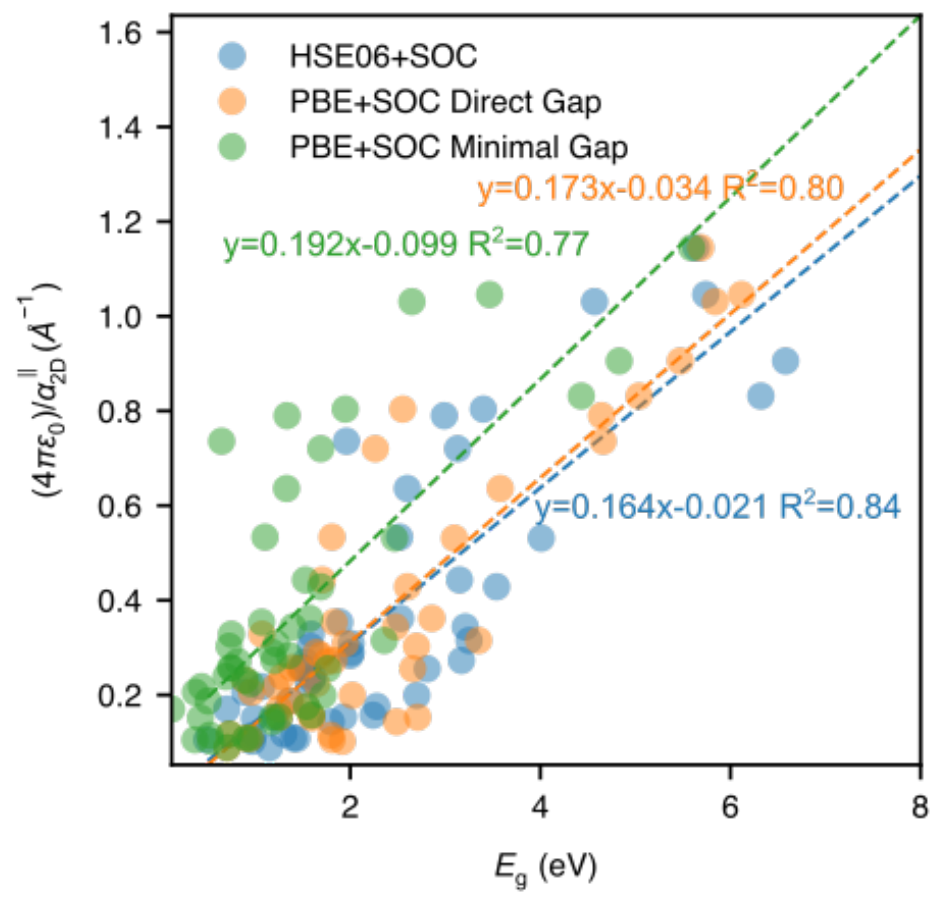

Figure 14: Relation between $1 / \alpha_{2 \mathrm{D}}^{\|}$and various choices of $E_{\mathrm{g}}$ : minimal gap from HSE06 (blue), minimal gap from PBE (orange) and direct gap from PBE (green). The linear regression results are shown as broken lines. 
transition. In this sense, $\alpha_{2 \mathrm{D}}^{\|}$is mostly like to be associated with the minimal, not direct bandgap, as also observed in the original Moss relation. We also examine the validity of such statement based on the analysis of a different database ${ }^{22}$ as will be discussed in the following sections.

\section{S5 Using a different dataset of 2D materials}

\section{S5.1 Validation of the universal description of 2D polarizabilities}

a

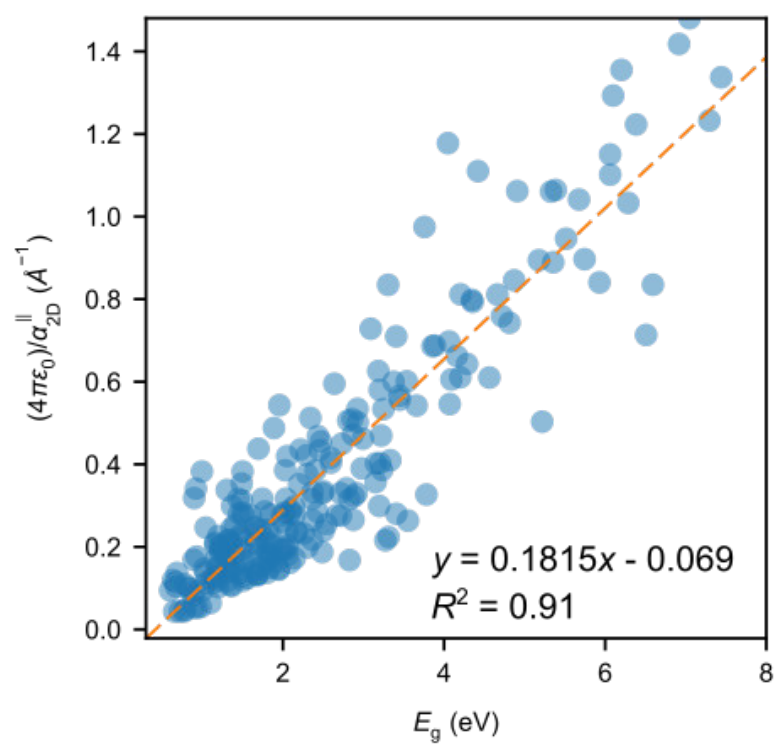

b

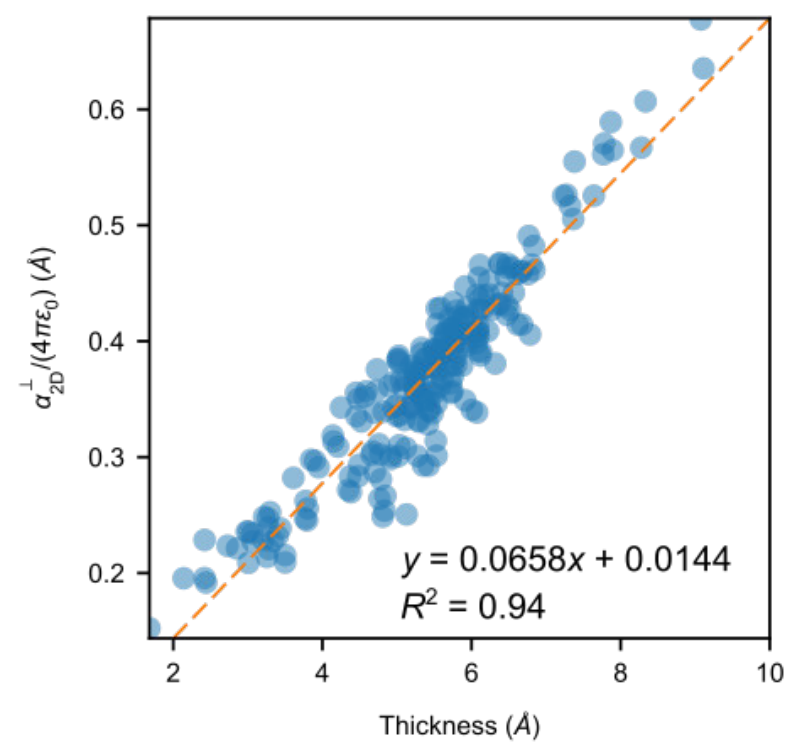

Figure 15: Validation of the linear relation between $\mathbf{a}, 1 / \alpha_{2 \mathrm{D}}^{\|}-E_{\mathrm{g}}(\mathrm{HSE})$ and $\mathbf{b}, \alpha_{2 \mathrm{D}}^{\perp}-\delta_{\mathrm{cov}}$ from Ref. 22 corresponding to main text Figure $2 \mathbf{c}$ and $2 \mathbf{d}$.

Due to time-consuming simulations and significant increment in memory overload, high-accurate calculations at hybrid HSE06 level is limited to about 55 compounds. It is desirable to validate our proposed relations on an even larger scale database. We select over 248 semiconducting 2D materials from Ref. 22 with a GW bandgap larger than $0.05 \mathrm{eV}$ and extracted the 2D polarizabilities calculated on the PBE level. The proposed linear relations between $1 / \alpha_{2 \mathrm{D}}^{\|}-E_{\mathrm{g}}(\mathrm{HSE})$ and $\alpha_{2 \mathrm{D}}^{\perp}-\delta_{\text {cov }}$ are also valid, as shown in Figure 15. Excellent linear correlation is observed in both cases with the $R^{2}$ coefficient larger than 0.9 which indicates the existence of a universal descrip- 
tion of 2D dielectric nature through the proposed relations with the $2 \mathrm{D}$ polarizabilities. We note that the slope of the linear regression is slightly different from the one proposed from the dielectric response at the HSE06 level.

\section{S5.2 Choice of bandgap}

Next we investigate the influence of choice of $E_{\mathrm{g}}$ on the regression of $\alpha_{2 \mathrm{D}}^{\|}-E_{\mathrm{g}}$ relation. Figure 16 shows $1 / \alpha_{2 \mathrm{D}}^{\|}$from Ref. 22 as a function of minimal and direct bandgap calculated on PBE, HSE06 and GW levels. We observe, although the regression $R^{2}$ coefficient in all cases are around 0.9 , the $\alpha_{2 \mathrm{D}}^{\|}-E_{\mathrm{g}}$ is better described using the HSE and GW bandgaps than the PBE bandgaps. On the other hand, using indirect or minimal bandgaps on the same level gives almost identical regression slope. The observations are in good agreement with our calculations on the HSE level discussed in Section S4. In combination with the physical contribution of $E_{\mathrm{g}}$ to the dielectric screening, we conclude that the minimal bandgap should be used for quantitative prediction of the in-plane 2D polarizability. The prediction is greatly improved when more accurate theory level for bandgap is used (for instance, HSE and GW).
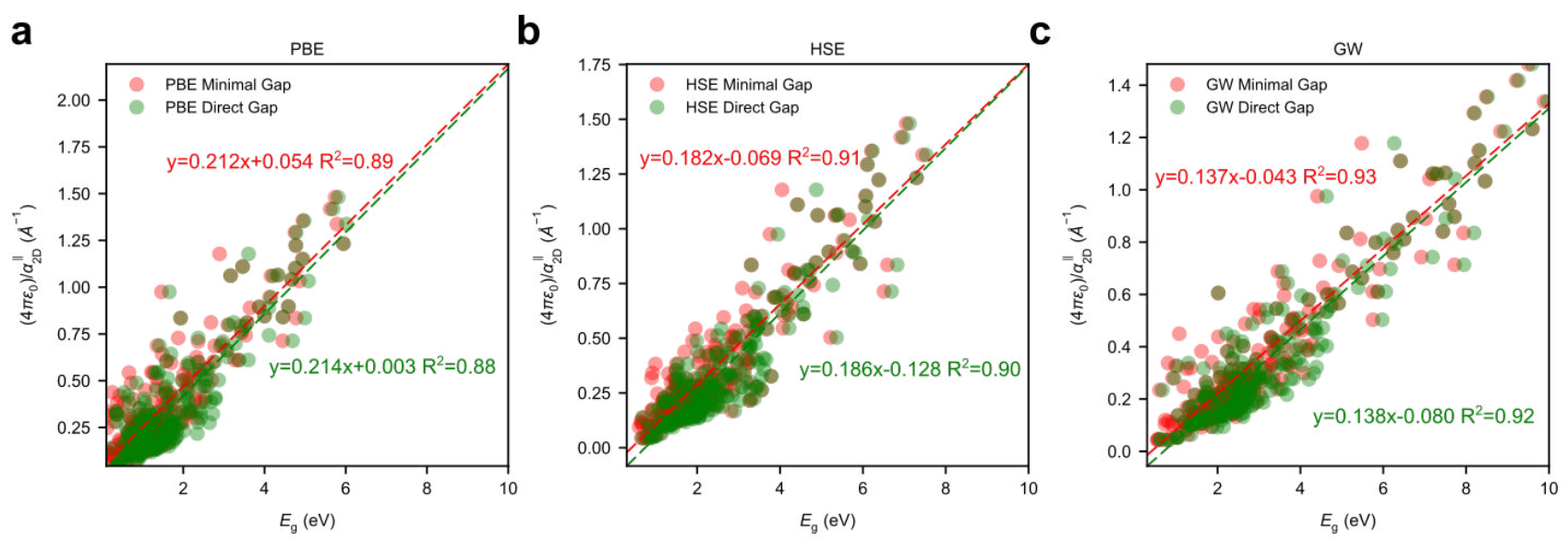

Figure 16: $\alpha_{2 \mathrm{D}}^{\|}$as function of minimal and direct $E_{\mathrm{g}}$ calculated on different theoretical levels: a, PBE, b, HSE and c, GW of Ref. 22.

For 2D materials, the exciton effect plays an important role in determining the experimentally accessible bandgap. ${ }^{9,23-25}$ The experimentally observed optical bandgap $E_{\mathrm{g}}^{\mathrm{opt}}$, is usually lower 
than the direct bandgap from band structure $E_{\mathrm{g}}^{\text {dir }}$ by the exciton binding energy $E_{\mathrm{b}}$, which is at the $10^{-1}$ to $10^{1} \mathrm{eV}$ for different $2 \mathrm{D}$ materials due to the attenuated dielectric screening. Next we examine the relation between $\alpha_{2 \mathrm{D}}^{\|}$and $E_{\mathrm{g}}^{\mathrm{opt}}$ from Ref. 22 with $E_{\mathrm{g}}^{\mathrm{opt}}=E_{\mathrm{g}}^{\mathrm{dir}, \mathrm{QP}}-E_{\mathrm{b}}^{\mathrm{BSE}}$, where $E_{\mathrm{g}}^{\mathrm{dir}, \mathrm{QP}}$ is the direct quasi-particle bandgap calculated using $\mathrm{G}_{0} \mathrm{~W}_{0}$ method and $E_{\mathrm{b}}^{\mathrm{BSE}}$ is the exciton binding energy calculated using the Bethe-Salpethe equation. Figure 17 shows $\left(4 \pi \varepsilon_{0}\right) / \alpha_{2 \mathrm{D}}^{\|}$as a function of $E_{\mathrm{g}}^{\mathrm{opt}}$, with a linear regression slope of 0.154 and $R^{2}$ of 0.84 , similar to the relation between $\left(4 \pi \varepsilon_{0}\right) / \alpha_{2 \mathrm{D}}^{\|}$and $E_{\mathrm{g}}$ (from HSE06 level, see Figures 13 and 16). The roughly linear correlation between $\left(4 \pi \varepsilon_{0}\right) / \alpha_{2 \mathrm{D}}^{\|}$and $E_{\mathrm{g}}^{\mathrm{opt}}$ is not coincidental: in fact, theoretical analysis shows that the binding energy $E_{\mathrm{b}}$ is proportional to the direct bandgap $E_{\mathrm{g}},{ }^{8}$ taking into account that $\left(\alpha_{2 \mathrm{D}}^{\|}\right)^{-1} \propto E_{\mathrm{g}}$, we rationalize that $\left(\alpha_{2 \mathrm{D}}^{\|}\right)^{-1} \propto E_{\mathrm{g}}^{\mathrm{opt}}=E_{\mathrm{g}}^{\mathrm{dir}}-E_{\mathrm{b}}$. The slightly smaller linearity than the 2D Moss-like relation is caused from multiple approximations used. Nevertheless we show that $\left(4 \pi \varepsilon_{0}\right) / \alpha_{2 \mathrm{D}}^{\|}$can be equivalently predicted using the experimentally accessible optical bandgap.

\section{S5.3 Relation between 2D polarizabilities and other physical quantities}

The relatively large size of Ref. 22 database allows us to examine the relation between 2D polarizabilities and other physical quantities. We choose the following quantities for comparison, corresponding to Figures 18 to 20:

1. The effective carrier mass for electron $m_{e}^{*}$ and hole $m_{h}^{*}$

2. The quantum capacitance at the conduction band edge $C_{\mathrm{Q}}^{\mathrm{C}}$ and valence band edge $\left(C_{\mathrm{Q}}^{\mathrm{V}}\right)$.

3. The total atomic polarizabilities per area $\alpha_{2 \mathrm{D}}^{\mathrm{sum}}$.

The quantum capacitance $C_{\mathrm{Q}}(E)$ at certain energy level $E$ is calculated using the relation $C_{\mathrm{Q}}(E)=\operatorname{DOS}(E) e^{2}$, where $\operatorname{DOS}(E)$ is the density of states at the conduction or valence band edge (averaged by cell area). The DOS value is calculated at the energy level with a charge cutoff such that $\left|n_{2 \mathrm{D}}(E)\right|=5 \times 10^{13} \mathrm{~cm}^{-2}$, calculated by the relation of accumulated charge $n_{2 \mathrm{D}}(E)$ at 


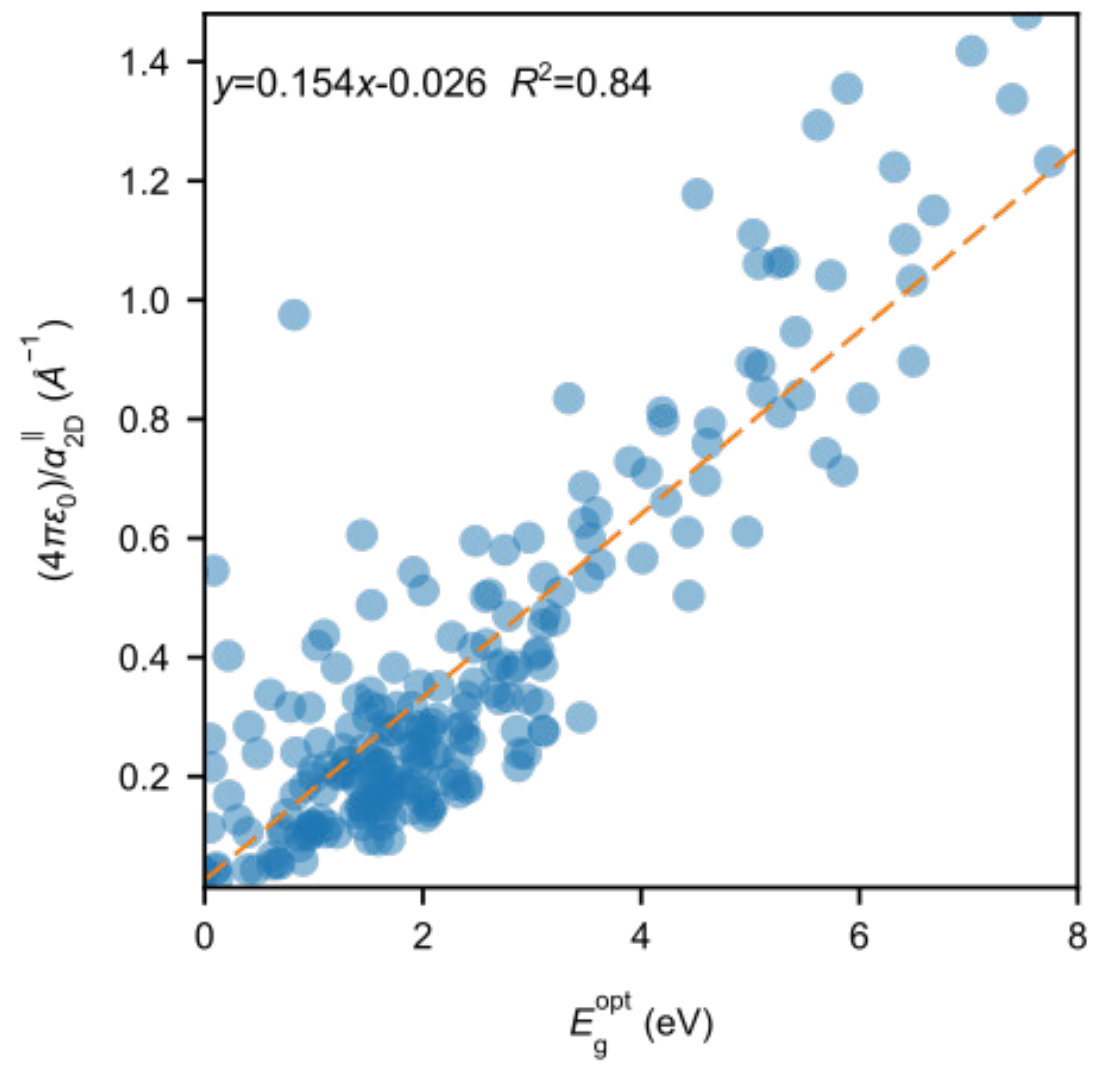

Figure 17: $\left(4 \pi \varepsilon_{0}\right) / \alpha_{2 \mathrm{D}}^{\|}$as a function of $E_{\mathrm{g}}^{\mathrm{opt}}$ from Ref. 22. 
a

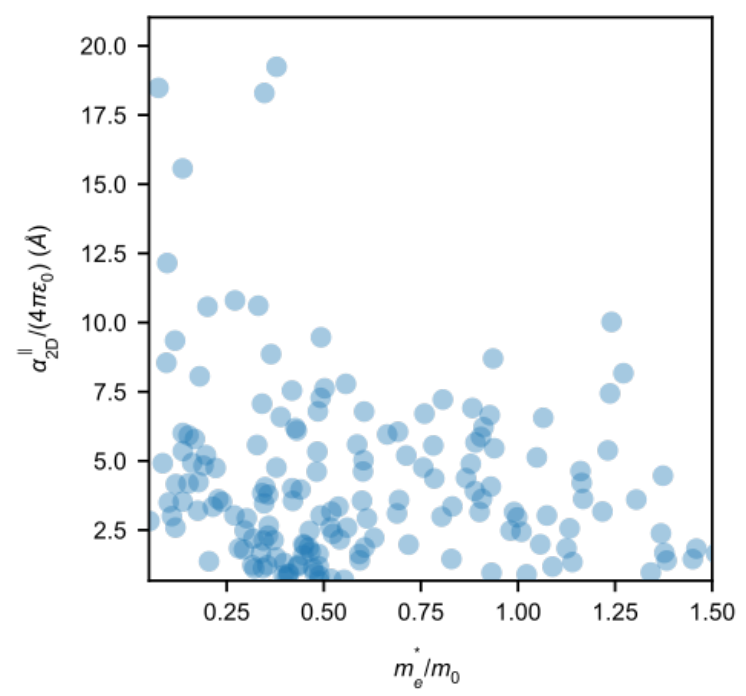

C

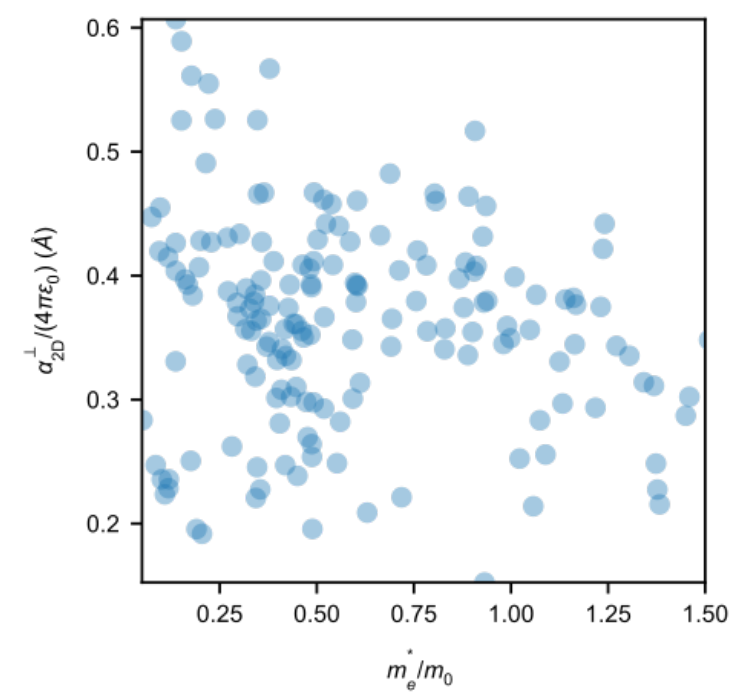

b

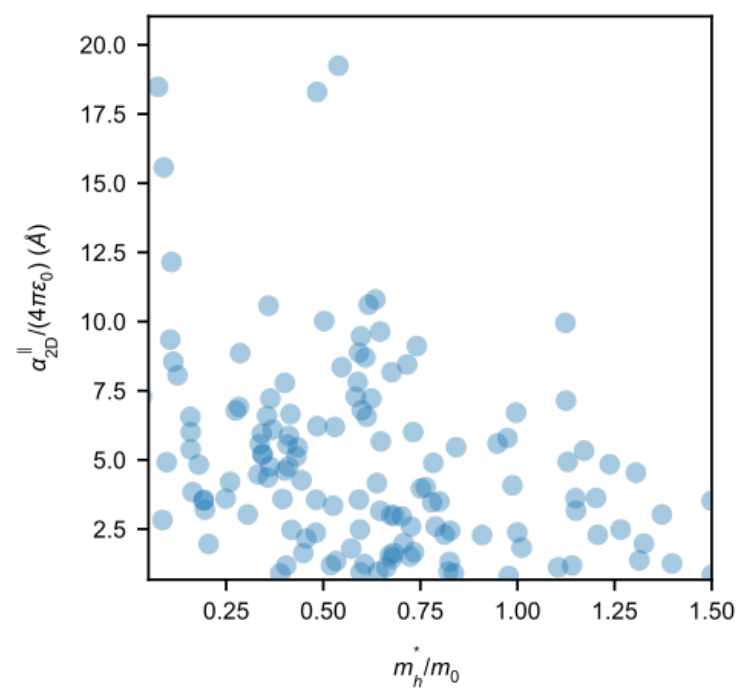

d

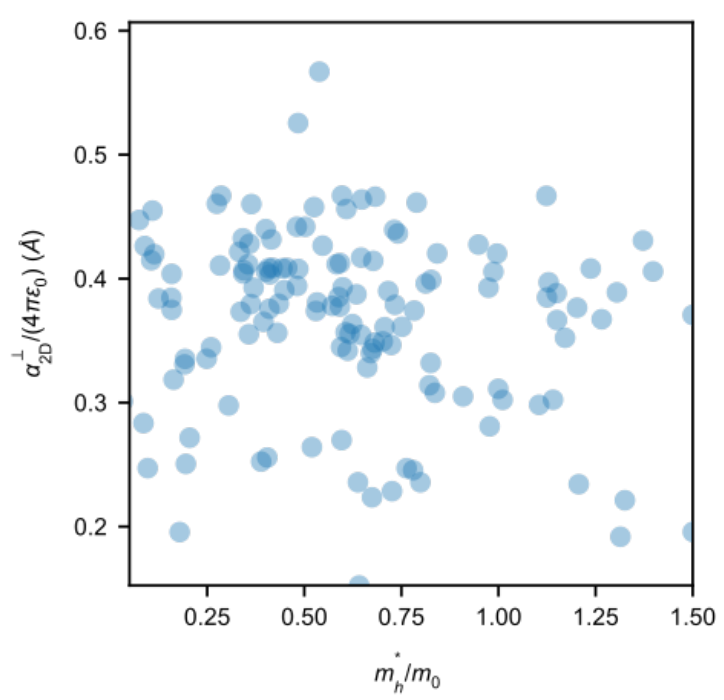

Figure 18: Relation between 2D polarizabilities and the effective carrier mass from Ref. 22. a. $\alpha_{2 \mathrm{D}}^{\|}$as a function of the electron mass $m_{e}^{*}$. b. $\alpha_{2 \mathrm{D}}^{\|}$as a function of the hole mass $m_{h}^{*}$. c. $\alpha_{2 \mathrm{D}}^{\perp}$ as a function of the electron mass $m_{e}^{*}$. d. $\alpha_{2 \mathrm{D}}^{\perp}$ as a function of the hole mass $m_{h}^{*}$. No apparent correlation between the 2D polarizabilities and the effective carrier masses is observed. 

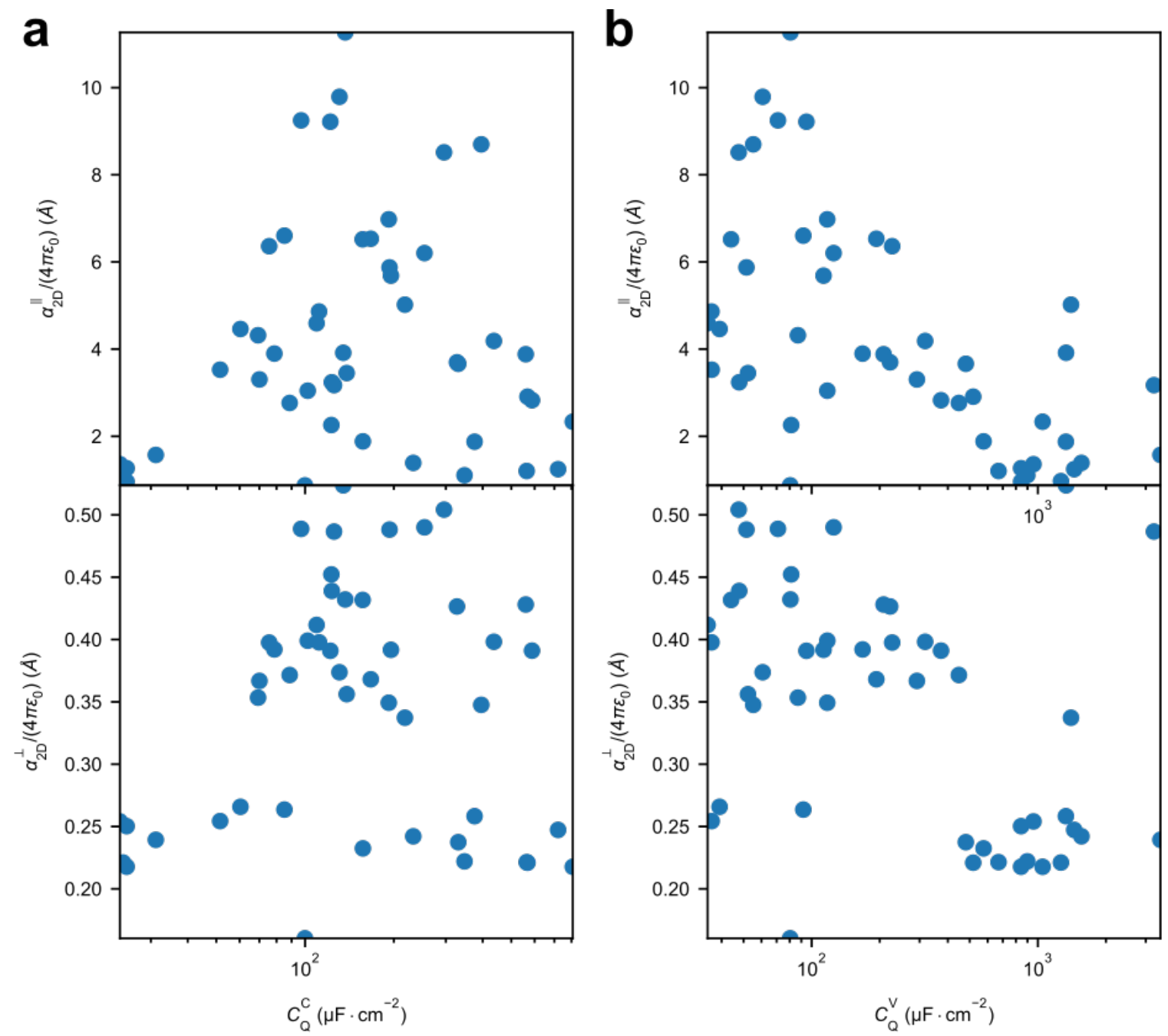

Figure 19: Relation between the 2D polarizabilities with the quantum capacitance. a $\alpha_{2 \mathrm{D}}^{\|}$(top) and $\alpha_{2 \mathrm{D}}^{\perp}$ (bottom) as functions of the quantum capacitance of the conduction band edge, $C_{\mathrm{Q}}^{\mathrm{C}}$. $\mathbf{b}$ $\alpha_{2 \mathrm{D}}^{\|}$(top) and $\alpha_{2 \mathrm{D}}^{\perp}$ (bottom) as functions of the quantum capacitance of the valence band edge, $C_{\mathrm{Q}}^{\mathrm{V}}$. Similar to the case of effective carrier mass, no apparent correlation between 2D polarizabilities and the quantum capacitance can be found. 

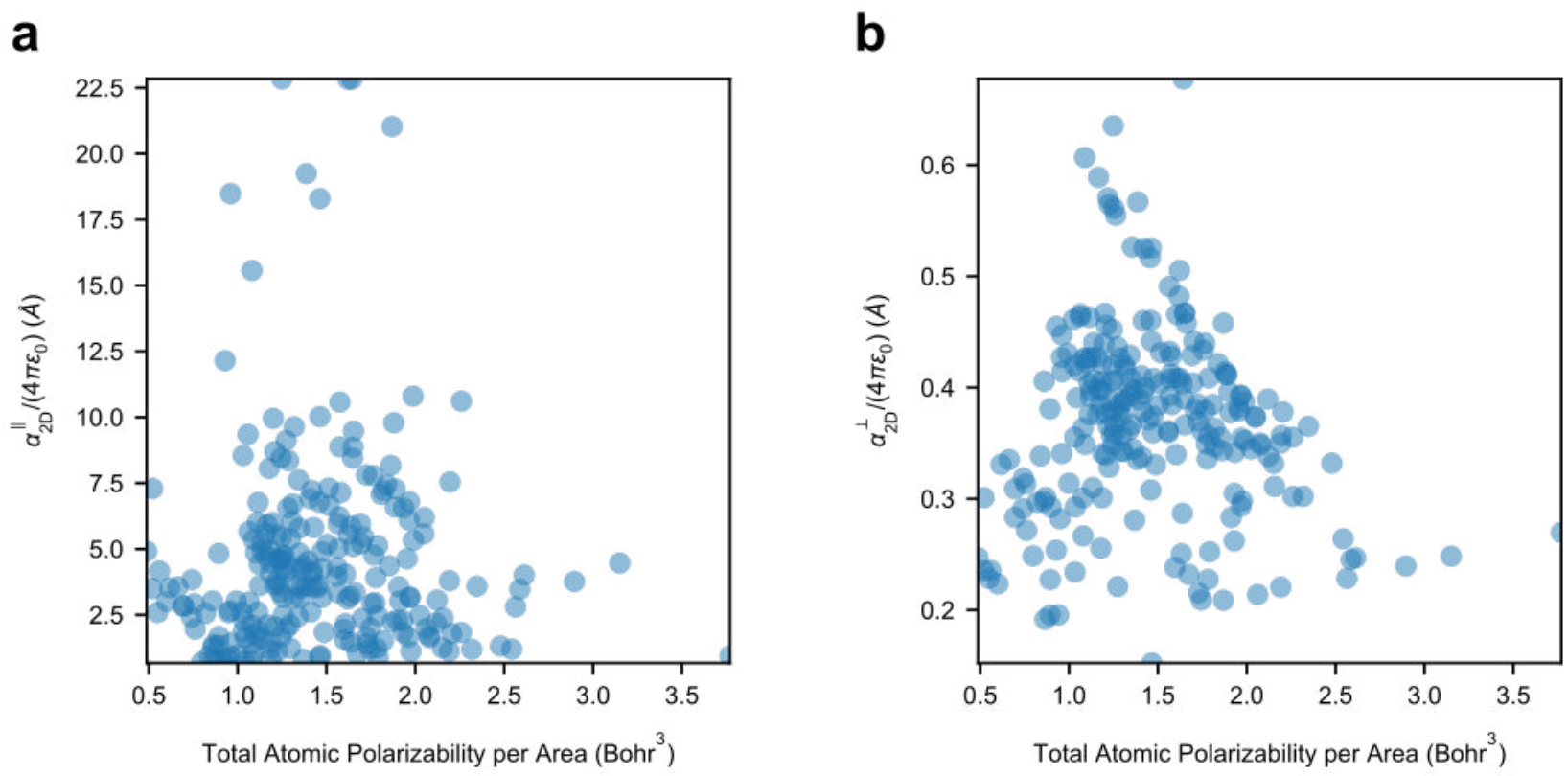

Figure 20: Relation between the 2D polarizabilities (a. $\alpha_{2 \mathrm{D}}^{\|}$and b. $\alpha_{2 \mathrm{D}}^{\perp}$ ) with the total atomic polarizability per area.

CB or VB:

$$
\left|n_{2 \mathrm{D}}(E)\right|=\left|\int_{E_{\mathrm{BE}}}^{E} \operatorname{DOS}\left(E^{\prime}\right) d E^{\prime}\right|
$$

where $E_{\mathrm{BE}}$ is the energy of the $\mathrm{CB}$ or VB band edge.

The total polarizability $\alpha_{2 \mathrm{D}}^{\mathrm{sum}}$ is calculated by the summation of the atomic polarizabilities $\alpha_{2 \mathrm{D}}^{\text {atom } 26}$ of individual atoms per area $A$, such that:

$$
\alpha_{2 \mathrm{D}}^{\mathrm{sum}}=\frac{\sum_{i} \alpha_{i}^{\text {atom }}}{A}
$$

From Figures 18 to 20 we can see that none of the above quantities have apparent relation with the $2 \mathrm{D}$ polarizabilities, as compared with the bandgap and covalent thickness proposed in the main text. 


\section{S6 More discussion about the relation between 2D and 3D prop- erties}

\section{S6.1 Comparing 2D and 3D Moss relations}

The 2D Moss-like relation $\alpha^{\|} \propto E_{g}^{-1}$ is similar to the 3D Moss relation $\varepsilon \propto E_{g}^{-1 / 2}$, with a different power law. Such difference in the power law can indeed be explained by modern theory of dielectric properties. From the 2D material to a bulk covalent semiconductor, the wave function becomes periodic in all directions. Considering only one pair of valence-conduction transition and uniform effective mass $m^{*}$, extending the approach Eq. S25 to the bulk material, and using the Bloch presentation for wave functions in all dimensions, we get: ${ }^{8}$

$$
\begin{aligned}
\varepsilon_{\text {bulk }}-1 & \propto \int d^{3} \mathbf{k} \frac{1}{\left(E_{\mathrm{g}}+\frac{\hbar^{2} k^{2}}{m^{*}}\right)^{2}} \\
& =\int_{0}^{k_{\mathrm{BZ}}} \frac{4 \pi k^{2}}{\left(E_{\mathrm{g}}+\frac{\hbar^{2} k^{2}}{m^{*}}\right)^{2}} d k
\end{aligned}
$$

where $k_{\mathrm{BZ}}$ is the boundary for the Brillouin Zone. The last step in Eq. S30 assumes the integral within the Brillouin Zone is equivalent to integral inside a sphere of k-space. Let $\hbar^{2} /\left(2 m^{*}\right)=\beta$, the integral becomes:

$$
\begin{aligned}
\varepsilon_{\mathrm{Bulk}} & \propto \frac{2 \pi \arctan \left(\sqrt{\beta k^{2} / E_{\mathrm{g}}}\right)}{\sqrt{E_{\mathrm{g}} \beta^{3}}}-\left.\frac{2 \pi k}{\beta\left(\beta+E_{\mathrm{g}} k^{2}\right)}\right|_{0} ^{k_{\mathrm{BZ}}} \\
& \propto 1 / \sqrt{E_{\mathrm{g}}}
\end{aligned}
$$

when $\varepsilon_{\mathrm{Bulk}} \gg 1$. since generally $\hbar^{2} k_{\mathrm{BZ}}^{2} /\left(2 m^{*}\right) \gg E_{\mathrm{g}} \cdot{ }^{27}$ The final result $\varepsilon_{\mathrm{Bulk}} \propto E_{\mathrm{g}}{ }^{-1 / 2}$ recovers the original Moss relation for bulk semiconductors. 


\section{S6.2 Static 2D polarizability and 2D plasma frequency}

A common approach for describing the bulk dielectric function of bulk semiconductors is via the Lorentz oscillator model, where the dielectric function is dominated by the plasma frequencies $\omega_{3 \mathrm{D}}^{\mathrm{p}}$ and bandgap $E_{\mathrm{g}}$ of individual oscillators. ${ }^{28}$ At zero optical frequency and the static limit, the dielectric constant for single oscillator is:

$$
\varepsilon_{3 \mathrm{D}}=1+\frac{\hbar^{2}\left(\omega_{3 \mathrm{D}}^{\mathrm{p}}\right)^{2}}{E_{\mathrm{g}}^{2}}
$$

where $\omega_{3 \mathrm{D}}^{\mathrm{p}}=\sqrt{\frac{e^{2} n_{3 \mathrm{D}}}{\varepsilon_{0} m_{e}}}$, where $n_{3 \mathrm{D}}$ is the $3 \mathrm{D}$ number density of valence electrons. Combine Eq. S32 with main text Eq. 2a, we get:

$$
\begin{aligned}
\alpha_{2 \mathrm{D}}^{\|} & =\frac{e^{2} n_{3 \mathrm{D}} L}{m_{e} E_{\mathrm{g}}^{2}} \\
& =\frac{e^{2} n_{2 \mathrm{D}}}{m_{e} E_{\mathrm{g}}^{2}} \\
& =\varepsilon_{0} \frac{\hbar^{2}\left(\omega_{2 \mathrm{D}}^{\mathrm{p}}\right)^{2}}{E_{\mathrm{g}}^{2}}
\end{aligned}
$$

where $n_{2 \mathrm{D}}=n_{3 \mathrm{D}} L$ is the $2 \mathrm{D}$ number density of valence electrons and $\omega_{2 \mathrm{D}}^{p}=\omega_{3 \mathrm{D}}^{p} \sqrt{L}$ is the $2 \mathrm{D}$ plasma frequency at static limit, ${ }^{29}$ as discussed in the main text. Apparently $n_{2 \mathrm{D}}$ and $\omega_{2 \mathrm{D}}^{p}$ defines the superlattice-independent 2D quantity $\alpha_{2 \mathrm{D}}^{\|}$, while its $3 \mathrm{D}$ counterpart $\varepsilon_{3 \mathrm{D}}$ is dependent on $L$. By defining the $2 \mathrm{D}$ valence charge density $\sigma_{2 \mathrm{D}}^{\mathrm{v}}=n_{2 \mathrm{D}} e$, we have also calculated $\alpha_{2 \mathrm{D}}^{\|}$as a function of $\sigma_{2 \mathrm{D}}^{\mathrm{v}} / E_{\mathrm{g}}^{2}$ using Ref. 22 database, as shown in Figure 21. It can be seen that, a large number of materials are close to the theoretical value of $\alpha_{2 \mathrm{D}}^{\|}=\frac{e^{2} n_{3 \mathrm{D}} L}{m_{e} E_{\mathrm{g}}^{2}}$ (broken line). However there are also many violations to this simple relation, making such model not suitable for quantitative prediction of the 2D dielectric nature, due to the oversimplification of single Lorentz oscillator. Nevertheless, this example shows excellently how the quantities in both dimensions are related to each other. 


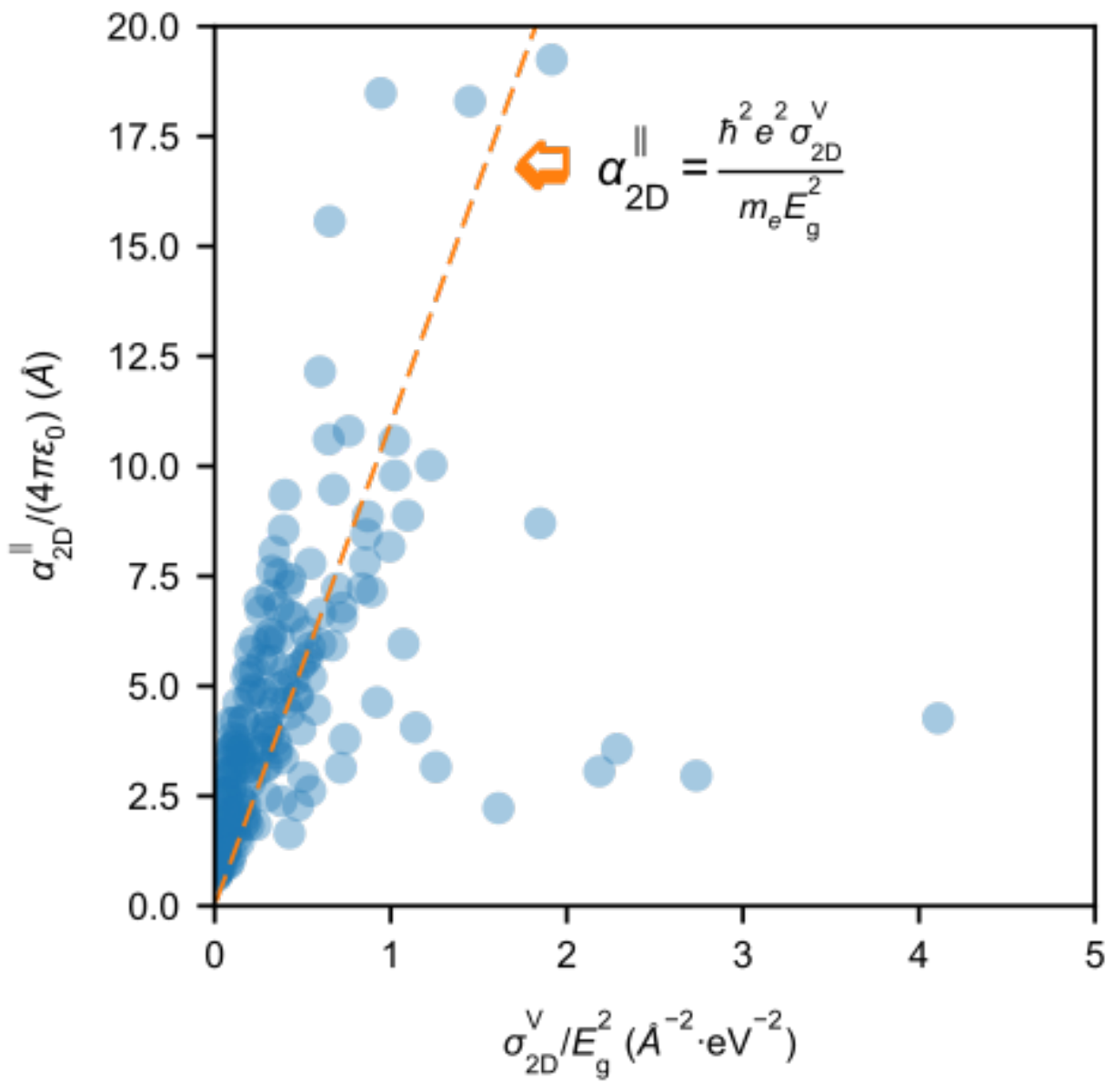

Figure 21: Calculated $\alpha_{2 \mathrm{D}}^{\|}$as a function of $\sigma_{2 \mathrm{D}}^{\mathrm{v}} / E_{\mathrm{g}}^{2}$ using data from Ref. 22. The broken line shows the theoretical prediction from single-oscillator model. 


\section{S6.3 The relation between 2D and 3D physical quantities}

As schematically shown in Figure 22, the physical quantities related to the dielectric properties can be categorized into (i) strictly 2D (microscopic), (ii) strictly 3D (macroscopic) and (iii) valid both $2 \mathrm{D}$ and $3 \mathrm{D} . \alpha_{2 \mathrm{D}}$ and $\varepsilon$ are the starting point for the strictly $2 \mathrm{D}$ and $3 \mathrm{D}$ quantities, which require distinct definitions when dimensionality changes. Such quantities include (but not limited to):

1. The densities $n_{2 \mathrm{D}}$ and $n_{3 \mathrm{D}}$ for charge, polarization, electronic states, etc.

2. The plasma frequencies $\omega_{2 \mathrm{D}}^{\mathrm{p}}$ and $\omega_{2 \mathrm{D}}^{\mathrm{p}}{ }^{29}$ (see Section S6.2 and Figure 21).

3. The optical conductivity $\sigma_{2 \mathrm{D}}$ and $\sigma_{3 \mathrm{D}} \cdot{ }^{3,30}$

These quantities have distinct units in both dimensions, and related by $L$ (for density and optical conductivity) or $\sqrt{L}$ (for plasma frequency), which requires prudent interpretation of theoretical and experimental results. For instance, the experimentally observed "dielectric constant" of monolayer 2D materials ${ }^{31-34}$ would be questionable without considering the effect of mixed medium. Instead, the 2D slab polarizability, either transformed from the vacuum-containing macroscopic dielectric constant, or predicted from the bandgap and geometry as proposed here, will be a better descriptor for the true 2D dielectric nature. There are also dimension-independent quantities that are valid for both 2D and 3D systems, for instance the bandgap $E_{\mathrm{g}}$, exciton binding energy $E_{\mathrm{b}}$, Bohr radius $r_{\mathrm{B}}$ of the exciton as well as the Hamaker constant of van der Waals interaction $A_{\mathrm{H}}$. All these quantities are well-defined and can be measured in both dimensions, while their relation with the dielectric property varies with dimensionality. The well-known examples are the different Wannier-Mott laws for exciton binding energy, ${ }^{7}$ the dielectric-bandgap relation proposed here, and the distinct power laws for van der Waals interactions. ${ }^{35}$ To get a accuracy description of dielectric-related properties of the 2D materials and their heterostructures, one has to distinguish between the $2 \mathrm{D}$ and $3 \mathrm{D}$ properties, and choose a suitable relation with the dimension-dependent and dimension-independent quantities. 


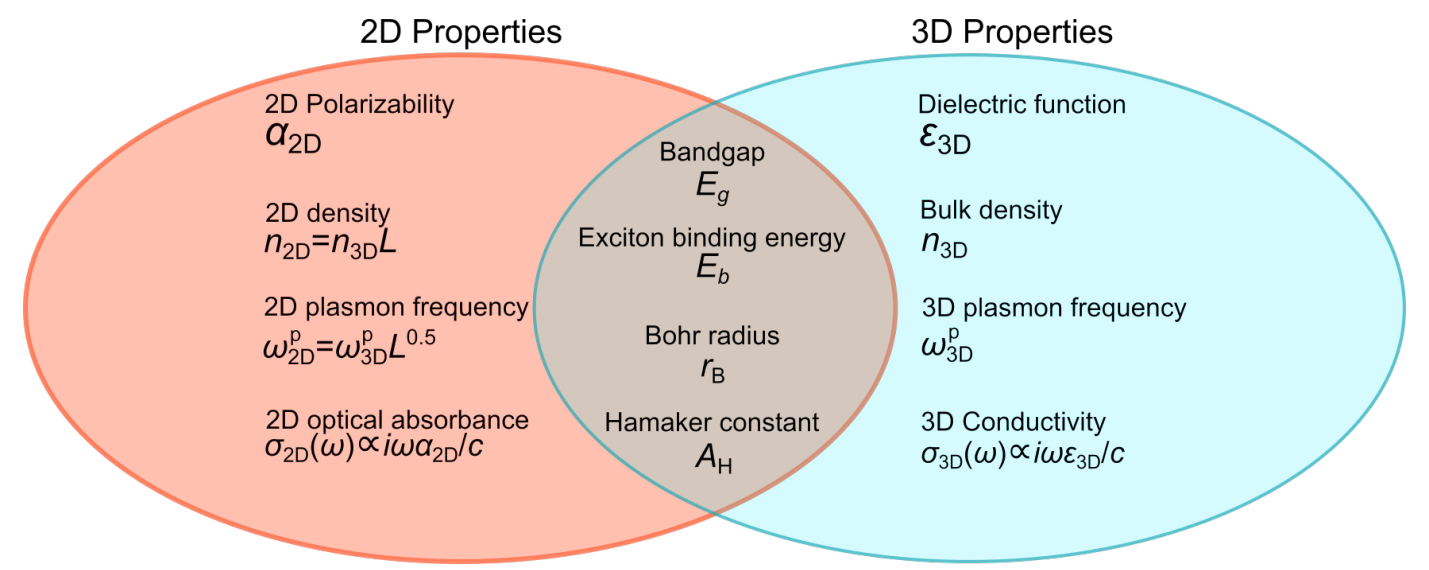

Figure 22: Dielectric-related physical quantities in both 2D (red circle) and 3D (cyan circle) systems. The dimension-dependent quantities can be related with $\alpha_{2 \mathrm{D}}$ and $\varepsilon$, respectively. The intersection between the circles present the quantities are well-defined in both dimensions, but may have a different scaling relation with others quantities.

\section{S7 More discussions about the dielectric anisotropy}

\section{S7.1 Choice of materials for main text Figure 5}

The dielectric anisotropy $\eta$ proposed in the main text is also applied to other dimensions. Similar to the case of $2 \mathrm{D}$ and bulk layered materials, $\epsilon$ is used to compare the anisotropy when the material is periodic in all dimensions (bulk covalent materials), while the polarizability $\alpha$ is used for reduced dimensional materials.

The following types materials are chosen for comparison:

- Bulk covalent materials.

The list of materials and bandgap are chosen according to Ref. 36, including IV-IV, III-V, II-VI, and IV-VI semiconductors. All these materials have isotropic dielectric properties.

\section{- Planar OSc}

The planar OScs include metal phthalocyanines, disk-like polycyclic aromatic hydrocarbon (PAHs), and benzene derivatives. The dimensionality of these materials are close to 2D materials due to their planar shape. The bandgap values (mostly at B3LYP density functional with $6-31 \mathrm{G}^{* *}$ basis sets) are extracted from the NIST Computational Chemistry Comparison 
and Benchmark Database (http://cccdb.nist.gov, Release 19, April 2018) and the polarizability values are obtained from Refs. 37,38 .

- Carbon nanotubes (CNT)

Like 2D materials, CNTs are periodic along the 1D directions, and should be treated in a similar way to get the polarizability proportional to [Length $]^{2} .{ }^{12}$ Semiconducting zigzag and armchair CNTs are considered, with their electronic properties obtained from Refs 12,39. and dielectric properties obtained from Refs. 12,40,41.

\section{- Linear OSc}

We choose the linear polyacenes (linear PAHs from benzene to nonacene) and zigzag polyacetylene (1-9 repeating units) as model systems of linear OScs. The bandgaps are obtained from 42 and the polarizabilities are obtained from 43.

\section{- Fullerenes}

The bandgap of fullerenes $\left(\mathrm{C}_{n}\right.$ where $n=60 * m^{2}$ where $\left.m=1 \sim 7\right)$ are taken from 44 and the polarizabilities are taken from 45. All these materials have isotropic polarizability due to the high symmetry.

\section{S7.2 Explanation for the separation between $2 D$ and 3D regimes in main text Figure 5}

In this section we give an analytical explanation for the separation between the dielectric anisotropy indices of 2D and their bulk counterparts. From main text Eqs. 2a, $2 \mathrm{~b}$ and 8, $\eta_{\text {Bulk }}$ of a bulk layered 
material is expressed as:

$$
\begin{aligned}
\eta_{\text {Bulk }} & =\frac{\varepsilon_{\text {Bulk }}^{\perp}}{\varepsilon_{\text {Bulk }}^{\|}} \\
& =\frac{1}{\left(1+\frac{\alpha_{\text {Bulk }}^{\|}}{\varepsilon_{0} L_{\mathrm{Bulk}}}\right)\left(1-\frac{\alpha_{\mathrm{Bulk}}^{\perp}}{\varepsilon_{0} L_{\mathrm{Bulk}}}\right)} \\
& =\frac{1}{\left[1-\left(\frac{\alpha_{\mathrm{Bulk}}^{\perp}}{\alpha_{\mathrm{Bulk}}^{\|}}\right)\left(\frac{\alpha_{\mathrm{Bulk}}^{\|}}{\varepsilon_{0} L_{\mathrm{Bulk}}}\right)\right]\left[1+\left(\frac{\alpha_{\mathrm{Bulk}}^{\|}}{\varepsilon_{0} L_{\mathrm{Bulk}}}\right)\right]}
\end{aligned}
$$

Name take the fact that $\alpha_{\mathrm{Bulk}}^{\|} \approx \alpha_{2 \mathrm{D}}^{\|}$(main text Figure $3 \mathrm{~b}$ ), we name $\alpha_{\mathrm{Bulk}}^{\|} / \epsilon_{0} L_{\mathrm{Bulk}} \approx \alpha_{2 \mathrm{D}}^{\|} / \epsilon_{0} L_{\mathrm{Bulk}}$ as $\gamma$. Furthermore we name $\alpha_{\mathrm{Bulk}}^{\perp} / \alpha_{\mathrm{Bulk}}^{\|}$as $\eta_{2 \mathrm{D}}$, that $\hat{\eta}_{2 \mathrm{D}}=\alpha_{\mathrm{Bulk}}^{\perp} / \alpha_{\mathrm{Bulk}}^{\|} \geq \alpha_{2 \mathrm{D}}^{\perp} / \alpha_{2 \mathrm{D}}^{\|}=\eta_{2 \mathrm{D}}$, and Eq. S34 is reduced to:

$$
\eta_{\mathrm{Bulk}}=\frac{1}{\left(1-\hat{\eta}_{2 \mathrm{D}} \gamma\right)(1+\gamma)}
$$

The minimal value for $\eta_{\text {Bulk }}$ when $\gamma>0$ is obtained by solving:

$$
\frac{\partial \eta_{\text {Bulk }}}{\partial \gamma}=\frac{2 \hat{\eta}_{2 \mathrm{D}} \gamma+\hat{\eta}_{2 \mathrm{D}}-1}{(\gamma+1)^{2}\left(1-\hat{\eta}_{2 \mathrm{D}} \gamma\right)^{2}}=0
$$

which gives that $\eta_{\mathrm{Bulk}} \geq \frac{4 \hat{\eta}_{2 \mathrm{D}}}{\left(\hat{\eta}_{2 \mathrm{D}}+1\right)^{2}}$, where the minimal value is taken at $\gamma=\frac{1}{2}\left(\frac{1}{\hat{\eta}_{2 \mathrm{D}}}-1\right)$. Since $\frac{4 \hat{\eta}_{2 \mathrm{D}}}{\left(\hat{\eta}_{2 \mathrm{D}}+1\right)^{2}}$ monotonically increases when $0<\hat{\eta}_{2 \mathrm{D}}<1$, we get the comparison between the dielectric anisotropy indices between 2D materials and their bulk counterparts:

$$
\eta_{\mathrm{Bulk}} \geq \frac{4 \eta_{2 \mathrm{D}}}{\left(\eta_{2 \mathrm{D}}+1\right)^{2}} \geq \eta_{2 \mathrm{D}}
$$

Since $\gamma$ is actually $2 r_{0}^{\|} / L_{\text {Bulk }}$, the ratio between the in-plane screening length $r_{0}^{\|}$and inter-plane distance $L_{\mathrm{Bulk}}$, and in general $r_{0}^{\|} \gg L_{\mathrm{Bulk}}$, we can conclude that the case when $\eta_{\mathrm{Bulk}}=\eta_{2 \mathrm{D}}$ only happens when $\eta_{2 \mathrm{D}}$ is much smaller than 1 . Therefore the separation between the $2 \mathrm{D}$ and 3D regimes in main text Figure 5 is explained. 


\section{S8 Raw data from first principles calculations}

\section{S8.1 Quantities from first principles calculation}

Table S2 shows the parameters and results from the first principles calculations for the 2D materials studied. The 2D screening lengthes $r_{0}^{\|}$and $r_{0}^{\perp}$ can be obtained by multiplying $2 \pi$ to the columns $\alpha_{2 \mathrm{D}}^{\|} /\left(4 \pi \varepsilon_{0}\right)$ and $\alpha_{2 \mathrm{D}}^{\perp} /\left(4 \pi \varepsilon_{0}\right)$, respectively.

Table 2: Raw data of the materials calculated in this study.

\begin{tabular}{|c|c|c|c|c|c|c|c|c|c|}
\hline \multirow[t]{3}{*}{ Material } & \multirow[t]{3}{*}{$\mathrm{L}(\AA)$} & \multirow{3}{*}{$\begin{array}{l}\text { HSE06 } \\
E_{\mathrm{g}}^{\min } \\
(\mathrm{eV})\end{array}$} & \multirow{3}{*}{$\begin{array}{l}\text { PBE } \\
E_{\mathrm{g}}^{\min } \\
(\mathrm{eV})\end{array}$} & \multirow{3}{*}{$\begin{array}{l}\text { PBE } \\
E_{\mathrm{g}}^{\text {direct }} \\
(\mathrm{eV})\end{array}$} & \multirow[t]{3}{*}{$\varepsilon_{\mathrm{SL}}^{\mathrm{xx}}$} & \multirow[t]{3}{*}{$\varepsilon_{\mathrm{SL}}^{\mathrm{yy}}$} & \multirow[t]{3}{*}{$\varepsilon_{\mathrm{SL}}^{\mathrm{zZ}}$} & \multicolumn{2}{|c|}{$\alpha_{2 \mathrm{D}}^{\|} /\left(4 \pi \varepsilon_{0}\right) \alpha_{2 \mathrm{D}}^{\perp} /\left(4 \pi \varepsilon_{0}\right)$} \\
\hline & & & & & & & & $(\AA)$ & $(\AA)$ \\
\hline & & & & & & & & & \\
\hline $1 \mathrm{~T}-\mathrm{TiO}_{2}$ & 26.668 & 4.010 & 3.096 & 2.467 & 1.887 & 1.887 & 1.123 & 1.882 & 0.232 \\
\hline $2 \mathrm{H}-\mathrm{TiO}_{2}$ & 27.648 & 2.520 & 1.808 & 1.103 & 1.852 & 1.852 & 1.133 & 1.875 & 0.258 \\
\hline $1 \mathrm{~T}-\mathrm{TiSe}_{2}$ & 33.049 & 1.360 & 1.372 & 0.505 & 3.029 & 3.029 & 1.190 & 5.336 & 0.420 \\
\hline $1 \mathrm{~T}-\mathrm{ZrO}_{2}$ & 26.561 & 6.320 & 5.039 & 4.431 & 1.569 & 1.569 & 1.117 & 1.203 & 0.221 \\
\hline $1 \mathrm{~T}-\mathrm{ZrS}_{2}$ & 32.622 & 2.010 & 1.643 & 1.180 & 2.329 & 2.329 & 1.159 & 3.450 & 0.356 \\
\hline $1 \mathrm{~T}-\mathrm{ZrSe} \mathrm{e}_{2}$ & 34.056 & 0.890 & 0.961 & 0.371 & 2.794 & 2.794 & 1.172 & 4.862 & 0.398 \\
\hline $2 \mathrm{H}-\mathrm{ZrO}_{2}$ & 28.188 & 3.130 & 2.264 & 1.690 & 1.619 & 1.619 & 1.121 & 1.389 & 0.242 \\
\hline $2 \mathrm{H}-\mathrm{ZrSe}{ }_{2}$ & 33.692 & 1.500 & 1.382 & 0.738 & 2.448 & 2.448 & 1.190 & 3.882 & 0.428 \\
\hline $2 \mathrm{H}-\mathrm{ZrTe}_{2}$ & 35.904 & 0.900 & 1.216 & 0.284 & 3.171 & 3.171 & 1.207 & 6.203 & 0.490 \\
\hline $1 \mathrm{~T}-\mathrm{HfO}_{2}$ & 26.636 & 6.580 & 5.471 & 4.830 & 1.521 & 1.521 & 1.117 & 1.104 & 0.222 \\
\hline $1 \mathrm{~T}-\mathrm{HfS}_{2}$ & 32.558 & 2.010 & 1.949 & 1.224 & 2.250 & 2.250 & 1.204 & 3.239 & 0.439 \\
\hline $1 \mathrm{~T}-\mathrm{HfSe}_{2}$ & 33.916 & 1.070 & 1.215 & 0.435 & 2.702 & 2.702 & 1.180 & 4.594 & 0.412 \\
\hline $2 \mathrm{H}-\mathrm{HfO}_{2}$ & 28.167 & 3.400 & 2.552 & 1.948 & 1.555 & 1.555 & 1.124 & 1.244 & 0.247 \\
\hline $2 \mathrm{H}-\mathrm{HfS}_{2}$ & 32.678 & 1.890 & 1.831 & 1.068 & 2.087 & 2.087 & 1.177 & 2.827 & 0.391 \\
\hline $2 \mathrm{H}-\mathrm{HfSe}_{2}$ & 33.419 & 1.530 & 1.754 & 0.819 & 2.390 & 2.390 & 1.191 & 3.697 & 0.426 \\
\hline $2 \mathrm{H}-\mathrm{HfTe}_{2}$ & 35.629 & 0.700 & 1.251 & 0.121 & 3.072 & 3.072 & 1.208 & 5.875 & 0.488 \\
\hline $1 \mathrm{~T}-\mathrm{GeO}_{2}$ & 26.526 & 5.740 & 6.118 & 3.466 & 1.453 & 1.453 & 1.115 & 0.956 & 0.218 \\
\hline $1 \mathrm{~T}-\mathrm{GeS}_{2}$ & 31.883 & 1.580 & 2.697 & 0.726 & 2.302 & 2.302 & 1.169 & 3.303 & 0.367 \\
\hline $1 \mathrm{~T}-\mathrm{GeO}_{2}$ & 27.908 & 2.990 & 4.643 & 1.335 & 1.570 & 1.570 & 1.127 & 1.266 & 0.250 \\
\hline $1 \mathrm{~T}-\mathrm{SnO}_{2}$ & 27.147 & 4.570 & 5.840 & 2.649 & 1.449 & 1.449 & 1.114 & 0.970 & 0.221 \\
\hline
\end{tabular}


Table 2: Raw data of the materials calculated in this study.

\begin{tabular}{|c|c|c|c|c|c|c|c|c|c|}
\hline \multirow[t]{3}{*}{ Material } & \multirow[t]{3}{*}{$\mathrm{L}(\AA)$} & \multirow{3}{*}{$\begin{array}{l}\text { HSE06 } \\
E_{\mathrm{g}}^{\min } \\
(\mathrm{eV})\end{array}$} & \multirow{3}{*}{$\begin{array}{l}\text { PBE } \\
E_{\mathrm{g}}^{\min } \\
(\mathrm{eV})\end{array}$} & \multirow{3}{*}{$\begin{array}{l}\mathrm{PBE} \\
E_{\mathrm{g}}^{\text {direct }} \\
(\mathrm{eV})\end{array}$} & \multirow[t]{3}{*}{$\varepsilon_{\mathrm{SL}}^{\mathrm{xx}}$} & \multirow[t]{3}{*}{$\varepsilon_{\mathrm{SL}}^{\mathrm{yy}}$} & \multirow[t]{3}{*}{$\varepsilon_{\mathrm{SL}}^{\mathrm{zZ}}$} & \multicolumn{2}{|c|}{$\alpha_{2 \mathrm{D}}^{\|} /\left(4 \pi \varepsilon_{0}\right) \alpha_{2 \mathrm{D}}^{\perp} /\left(4 \pi \varepsilon_{0}\right)$} \\
\hline & & & & & & & & $(\AA)$ & $(\AA)$ \\
\hline & & & & & & & & & \\
\hline $1 \mathrm{~T}-\mathrm{SnS}_{2}$ & 32.793 & 2.530 & 2.859 & 1.574 & 2.059 & 2.059 & 1.166 & 2.764 & 0.372 \\
\hline $1 \mathrm{~T}-\mathrm{SnSe}_{2}$ & 34.077 & 1.490 & 1.466 & 0.751 & 2.437 & 2.437 & 1.169 & 3.897 & 0.392 \\
\hline $2 \mathrm{H}-\mathrm{SnO}_{2}$ & 28.938 & 1.960 & 4.661 & 0.647 & 1.590 & 1.590 & 1.124 & 1.359 & 0.254 \\
\hline $2 \mathrm{H}-\mathrm{SnS}_{2}$ & 32.873 & 1.590 & 1.072 & 0.750 & 2.164 & 2.164 & 1.180 & 3.045 & 0.399 \\
\hline $1 \mathrm{~T}-\mathrm{PbO}_{2}$ & 27.862 & 2.600 & 3.578 & 1.330 & 1.709 & 1.709 & 1.121 & 1.572 & 0.239 \\
\hline $\mathrm{BN}$ & 29.995 & 5.640 & 5.688 & 5.592 & 1.366 & 1.366 & 1.072 & 0.874 & 0.160 \\
\hline $\mathrm{C}_{2} \mathrm{~F}_{2}$ & 31.998 & 5.000 & 3.173 & 3.173 & 1.318 & 1.348 & 1.123 & 0.810 & 0.279 \\
\hline $\mathrm{P}_{4}$ & 27.097 & 1.600 & 0.888 & 0.895 & 2.894 & 3.115 & 1.196 & 4.084 & 0.353 \\
\hline $\mathrm{C}_{2} \mathrm{H}_{2}$ & 31.015 & 4.360 & 3.468 & 3.468 & 1.288 & 1.288 & 1.094 & 0.711 & 0.212 \\
\hline $1 \mathrm{~T}-\mathrm{NiO}_{2}$ & 26.112 & 3.170 & 1.828 & 1.198 & 2.763 & 2.763 & 1.129 & 3.663 & 0.237 \\
\hline $1 \mathrm{~T}-\mathrm{PdO}_{2}$ & 26.712 & 3.210 & 2.475 & 1.397 & 2.368 & 2.368 & 1.116 & 2.908 & 0.221 \\
\hline $1 \mathrm{~T}-\mathrm{PdS}_{2}$ & 30.361 & 1.800 & 2.487 & 1.178 & 3.888 & 3.888 & 1.169 & 6.978 & 0.349 \\
\hline $1 \mathrm{~T}-\mathrm{PtO}_{2}$ & 26.316 & 3.540 & 2.602 & 1.691 & 2.114 & 2.114 & 1.116 & 2.333 & 0.218 \\
\hline $1 \mathrm{~T}-\mathrm{PtS}_{2}$ & 30.239 & 2.700 & 2.022 & 1.714 & 3.086 & 3.086 & 1.163 & 5.020 & 0.337 \\
\hline $1 \mathrm{~T}-\mathrm{PdSe} \mathrm{e}_{2}$ & 31.080 & 0.970 & 1.917 & 0.534 & 4.958 & 4.958 & 1.178 & 9.789 & 0.374 \\
\hline $1 \mathrm{~T}-\mathrm{NiS}_{2}$ & 29.616 & 0.980 & 1.797 & 0.523 & 4.691 & 4.691 & 1.173 & 8.699 & 0.348 \\
\hline $1 \mathrm{~T}-\mathrm{PtSe}_{2}$ & 31.058 & 1.210 & 2.710 & 1.180 & 3.643 & 3.643 & 1.175 & 6.532 & 0.368 \\
\hline $\mathrm{Ga}_{2} \mathrm{Se}_{2}$ & 30.000 & 2.810 & 2.657 & 1.764 & 2.640 & 2.640 & 1.281 & 3.915 & 0.524 \\
\hline $\mathrm{Ga}_{2} \mathrm{~S}_{2}$ & 30.000 & 3.250 & 3.351 & 2.358 & 2.329 & 2.329 & 1.256 & 3.173 & 0.487 \\
\hline $\mathrm{CdCl}_{2}$ & 31.085 & 4.480 & 3.172 & 3.172 & 1.48 & 1.48 & 1.157 & 1.187 & 0.336 \\
\hline $\mathrm{CdI}_{2}$ & 35.281 & 3.150 & 1.706 & 1.528 & 1.804 & 1.804 & 1.192 & 2.257 & 0.452 \\
\hline $2 \mathrm{H}-\mathrm{MoS}_{2}$ & 32.296 & 2.240 & 1.594 & 1.594 & 3.475 & 3.475 & 1.183 & 6.361 & 0.398 \\
\hline $2 \mathrm{H}-\mathrm{MoSe}_{2}$ & 40.854 & 1.752 & 1.449 & 1.449 & 3.231 & 3.231 & 1.154 & 7.253 & 0.433 \\
\hline $2 \mathrm{H}-\mathrm{WS}_{2}$ & 32.271 & 2.280 & 1.540 & 1.540 & 3.214 & 3.214 & 1.180 & 5.686 & 0.392 \\
\hline $2 \mathrm{H}-\mathrm{WSe}_{2}$ & 32.965 & 1.930 & 1.253 & 1.253 & 3.485 & 3.485 & 1.197 & 6.519 & 0.432 \\
\hline $2 \mathrm{H}-\mathrm{WO}_{2}$ & 29.183 & 2.000 & 1.693 & 1.359 & 2.519 & 2.519 & 1.123 & 3.528 & 0.254 \\
\hline $2 \mathrm{H}-\mathrm{MoO}_{2}$ & 29.231 & 1.560 & 1.648 & 0.952 & 2.918 & 2.918 & 1.129 & 4.462 & 0.266 \\
\hline 2H-MoTe 2 & 34.061 & 1.440 & 0.946 & 0.946 & 4.412 & 4.412 & 1.220 & 9.248 & 0.489 \\
\hline
\end{tabular}


Table 2: Raw data of the materials calculated in this study.

\begin{tabular}{|c|c|c|c|c|c|c|c|c|c|}
\hline \multirow[t]{3}{*}{ Material } & \multirow[t]{3}{*}{$\mathrm{L}(\AA)$} & \multirow{3}{*}{$\begin{array}{l}\text { HSE06 } \\
E_{\mathrm{g}}^{\min } \\
(\mathrm{eV})\end{array}$} & \multirow{3}{*}{$\begin{array}{l}\text { PBE } \\
E_{\mathrm{g}}^{\min } \\
(\mathrm{eV})\end{array}$} & \multirow{3}{*}{$\begin{array}{l}\text { PBE } \\
E_{\mathrm{g}}^{\text {direct }} \\
(\mathrm{eV})\end{array}$} & \multirow[t]{3}{*}{$\varepsilon_{\mathrm{SL}}^{\mathrm{xx}}$} & \multirow[t]{3}{*}{$\varepsilon_{\mathrm{SL}}^{\mathrm{yy}}$} & \multirow[t]{3}{*}{$\varepsilon_{\mathrm{SL}}^{\mathrm{ZZ}}$} & \multicolumn{2}{|c|}{$\alpha_{2 \mathrm{D}}^{\|} /\left(4 \pi \varepsilon_{0}\right) \alpha_{2 \mathrm{D}}^{\perp} /\left(4 \pi \varepsilon_{0}\right)$} \\
\hline & & & & & & & & $(\AA)$ & $(\AA)$ \\
\hline & & & & & & & & & \\
\hline $2 \mathrm{H}-\mathrm{WTe}_{2}$ & 33.883 & 1.300 & 0.731 & 0.731 & 4.158 & 4.158 & 1.230 & 8.515 & 0.504 \\
\hline $2 \mathrm{H}-\mathrm{CrS}_{2}$ & 31.759 & 1.400 & 0.902 & 0.902 & 4.647 & 4.647 & 1.183 & 9.217 & 0.391 \\
\hline $2 \mathrm{H}-\mathrm{CrSe} \mathrm{e}_{2}$ & 32.446 & 1.150 & 0.704 & 0.704 & 5.364 & 5.364 & 1.201 & 11.268 & 0.432 \\
\hline $2 \mathrm{H}-\mathrm{CrO}_{2}$ & 28.027 & 0.990 & 1.596 & 0.424 & 3.961 & 3.961 & 1.134 & 6.604 & 0.264 \\
\hline $2 \mathrm{H}-\mathrm{TiS}_{2}$ & 32.199 & 1.610 & 1.284 & 0.692 & 2.634 & 2.634 & 1.184 & 4.187 & 0.398 \\
\hline $1 \mathrm{~T}-\mathrm{PtTe}_{2}$ & 32.005 & 0.490 & 1.809 & 0.366 & 4.726 & 4.726 & 1.200 & 9.490 & 0.424 \\
\hline $\mathrm{MAPbBr}_{3}$ & 23.018 & 3.163 & 2.444 & 2.444 & 1.608 & 1.778 & 1.527 & 1.265 & 0.632 \\
\hline
\end{tabular}




\section{References}

1. Rozzi, C. A.; Varsano, D.; Marini, A.; Gross, E. K. U.; Rubio, A. Exact Coulomb cutoff technique for supercell calculations. Phys. Rev. B 2006, 73, 205119.

2. Hüser, F.; Olsen, T.; Thygesen, K. S. How dielectric screening in two-dimensional crystals affects the convergence of excited-state calculations: Monolayer $\mathrm{MoS}_{2}$. Phys. Rev. B 2013, $88,245309$.

3. Matthes, L.; Pulci, O.; Bechstedt, F. Influence of out-of-plane response on optical properties of two-dimensional materials: First principles approach. Phys. Rev. B 2016, 94, 205408.

4. Laturia, A.; Van de Put, M. L.; Vandenberghe, W. G. Dielectric properties of hexagonal boron nitride and transition metal dichalcogenides: from monolayer to bulk. npj 2D Mater. Appl. 2018, 2, 6 .

5. Markel, V. A. Introduction to the Maxwell Garnett approximation: tutorial. J. Opt. Soc. Am. A 2016, 33, 1244.

6. Choi, J.-H.; Cui, P.; Lan, H.; Zhang, Z. Linear Scaling of the Exciton Binding Energy versus the Band Gap of Two-Dimensional Materials. Phys. Rev. Lett. 2015, 115, 066403.

7. Olsen, T.; Latini, S.; Rasmussen, F.; Thygesen, K. S. Simple Screened Hydrogen Model of Excitons in Two-Dimensional Materials. Phys. Rev. Lett. 2016, 116, 056401.

8. Jiang, Z.; Liu, Z.; Li, Y.; Duan, W. Scaling Universality between Band Gap and Exciton Binding Energy of Two-Dimensional Semiconductors. Phys. Rev. Lett. 2017, 118.

9. Pulci, O.; Marsili, M.; Garbuio, V.; Gori, P.; Kupchak, I.; Bechstedt, F. Excitons in twodimensional sheets with honeycomb symmetry. Phys. Status Solidi B 2014, 252, 72-77. 
10. Davies, P. Polarizabilities of long chain conjugated molecules. Trans. Faraday Soc. 1952, 48, $789-795$.

11. Sabirov, D. S. Polarizability as a landmark property for fullerene chemistry and materials science. RSC Adv. 2014, 4, 44996-45028.

12. Benedict, L. X.; Louie, S. G.; Cohen, M. L. Static polarizabilities of single-wall carbon nanotubes. Phys. Rev. B 1995, 52, 8541-8549.

13. Davies, J. H. The Physics of Low-dimensional Semiconductors: An Introduction; Cambridge University Press, 1997.

14. Adler, S. L. Quantum Theory of the Dielectric Constant in Real Solids. Phys. Rev. 1962, 126, $413-420$.

15. Hybertsen, M. S.; Louie, S. G. Ab initiostatic dielectric matrices from the density-functional approach. I. Formulation and application to semiconductors and insulators. Phys. Rev. B 1987, $35,5585-5601$.

16. Ihn, T. Semiconductor Nanostructures: Quantum states and electronic transport; Oxford University Press, 2009.

17. Fowler, P. Energy, polarizability and size of confined one-electron systems. Molecular Phys. 1984, 53, 865-889.

18. Maize, M. A.; Antonacci, M. A.; Marsiglio, F. The static electric polarizability of a particle bound by a finite potential well. American Journal of Physics 2011, 79, 222-225.

19. Heyd, J.; Peralta, J. E.; Scuseria, G. E.; Martin, R. L. Energy band gaps and lattice parameters evaluated with the Heyd-Scuseria-Ernzerhof screened hybrid functional. J. Chem. Phys. 2005, $123,174101$. 
20. Kumar, P.; Bhadoria, B. S.; Kumar, S.; Bhowmick, S.; Chauhan, Y. S.; Agarwal, A. Thickness and electric-field-dependent polarizability and dielectric constant in phosphorene. Phys.Rev. B 2016, 93, 195428.

21. Kumar, P.; Chauhan, Y. S.; Agarwal, A.; Bhowmick, S. Thickness and Stacking Dependent Polarizability and Dielectric Constant of Graphene-Hexagonal Boron Nitride Composite Stacks. J. Phys. Chem. C 2016, 120, 17620-17626.

22. Haastrup, S.; Strange, M.; Pandey, M.; Deilmann, T.; Schmidt, P. S.; Hinsche, N. F.; Gjerding, M. N.; Torelli, D.; Larsen, P. M.; Riis-Jensen, A. C. et al. The Computational 2D Materials Database: high-throughput modeling and discovery of atomically thin crystals. 2D Mater. 2018, 5, 042002 .

23. Arnaud, B.; Lebègue, S.; Rabiller, P.; Alouani, M. Huge Excitonic Effects in Layered Hexagonal Boron Nitride. Phys. Rev. Lett. 2006, 96, 026402.

24. Ramasubramaniam, A. Large excitonic effects in monolayers of molybdenum and tungsten dichalcogenides. Physical Review B 2012, 86, 115409.

25. Chernikov, A.; Berkelbach, T. C.; Hill, H. M.; Rigosi, A.; Li, Y.; Aslan, O. B.; Reichman, D. R.; Hybertsen, M. S.; Heinz, T. F. Exciton Binding Energy and Nonhydrogenic Rydberg Series in Monolayer $\mathrm{WS}_{2}$. Phys. Rev. Lett. 2014, 113, 076802.

26. Gould, T.; Bucko, T. C6 Coefficients and Dipole Polarizabilities for All Atoms and Many Ions in Rows 1-6 of the Periodic Table. J. Chem. Theory and Comput. 2016, 12, 3603-3613.

27. Finkenrath, $\mathrm{H}$. The moss rule and the influence of doping on the optical dielectric constant of semiconductors-II. Infrared Physics 1988, 28, 363-366.

28. Ketterson, J. B. The Physics of Solids; Oxford University Press, 2016.

29. Nazarov, V. U. Electronic excitations in quasi-2D crystals: what theoretical quantities are relevant to experiment? New J. Phys. 2015, 17, 073018. 
30. Bechstedt, F.; Matthes, L.; Gori, P.; Pulci, O. Infrared absorbance of silicene and germanene. Appl. Phys. Lett. 2012, 100, 261906.

31. Ning, M.-Q.; Lu, M.-M.; Li, J.-B.; Chen, Z.; Dou, Y.-K.; Wang, C.-Z.; Rehman, F.; Cao, M.S.; Jin, H.-B. Two-dimensional nanosheets of $\mathrm{MoS}_{2}$ : a promising material with high dielectric properties and microwave absorption performance. Nanoscale 2015, 7, 15734-15740.

32. Li, Y.; Chernikov, A.; Zhang, X.; Rigosi, A.; Hill, H. M.; van der Zande, A. M.; Chenet, D. A.; Shih, E.-M.; Hone, J.; Heinz, T. F. Measurement of the optical dielectric function of monolayer transition-metal dichalcogenides: $\mathrm{MoS}_{2}, \mathrm{MoSe}_{2}, \mathrm{WS}_{2}$, andWSe 2 . Phys. Rev. B 2014, 90, 205422.

33. Yao, J.; Koski, K. J.; Luo, W.; Cha, J. J.; Hu, L.; Kong, D.; Narasimhan, V. K.; Huo, K.; Cui, Y. Optical transmission enhacement through chemically tuned two-dimensional bismuth chalcogenide nanoplates. Nat. Commun. 2014, 5, 5670.

34. Wu, D.; Pak, A. J.; Liu, Y.; Zhou, Y.; Wu, X.; Zhu, Y.; Lin, M.; Han, Y.; Ren, Y.; Peng, H. et al. Thickness-Dependent Dielectric Constant of Few-Layer In2Se3 Nanoflakes. Nano Letters 2015, 15, 8136-8140.

35. Gobre, V. V.; Tkatchenko, A. Scaling laws for van der Waals interactions in nanostructured materials. Nat. Commun. 2013, 4, 2341.

36. Sze, S.; Ng, K. K. Physics of Semiconductor Devices; John Wiley \& Sons, Inc., 2006; pp 789-789.

37. Miller, K. J. Calculation of the molecular polarizability tensor. J. Am. Chem. Soc. 1990, 112, $8543-8551$.

38. Ramprasad, R.; Shi, N. Polarizability of phthalocyanine based molecular systems: A firstprinciples electronic structure study. Appl. Phys. Lett. 2006, 88, 222903. 
39. Matsuda, Y.; Tahir-Kheli, J.; Goddard, W. A. Definitive Band Gaps for Single-Wall Carbon Nanotubes. J. Phys. Chem. Lett. 2010, 1, 2946-2950.

40. Jensen, L.; Schmidt, O. H.; Mikkelsen, K. V.; Åstrand, P.-O. Static and Frequency-Dependent Polarizability Tensors for Carbon Nanotubes. J. Phys. Chem. B 2000, 104, 10462-10466.

41. Brothers, E. N.; Izmaylov, A. F.; Scuseria, G. E.; Kudin, K. N. Analytically Calculated Polarizability of Carbon Nanotubes: Single Wall, Coaxial, and Bundled Systems. J. Phys. Chem. C 2008, 112, 1396-1400.

42. Salzner, U.; Pickup, P. G.; Poirier, R. A.; Lagowski, J. B. Accurate Method for Obtaining Band Gaps in Conducting Polymers Using a DFT/Hybrid Approach. J. Phys. Chem. A 1998, 102, $2572-2578$.

43. Hinchliffe, A.; Nikolaidi, B.; Soscun Machado, H. Density functional studies of the dipole polarizabilities of the linear polyacenes benzene through nonacene. Open Chem. 2005, 3, 361369.

44. Lin, Y.-L.; Nori, F. Electronic structure of single- and multiple-shell carbon fullerenes. Phys. Rev. B 1994, 49, 5020-5023.

45. Martin, D.; Sild, S.; Maran, U.; Karelson, M. QSPR Modeling of the Polarizability of Polyaromatic Hydrocarbons and Fullerenes. J. Phys. Chem. C 2008, 112, 4785-4790. 